\title{
Heteroleptic Rare-Earth Tris(metallocenes) Containing a Dibenzocyclooctatetraene Dianion
}

Elizabeth R. Pugliese, Florian Benner, Ernesto Castellanos, Francis Delano, IV, and Selvan Demir*

Department of Chemistry, Michigan State University, 578 South Shaw

Lane, East Lansing, Michigan 48824, USA

Email: sdemir@chemistry.msu.edu

Inorg. Chem. 


\section{Table of Contents}

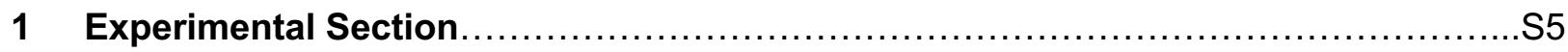

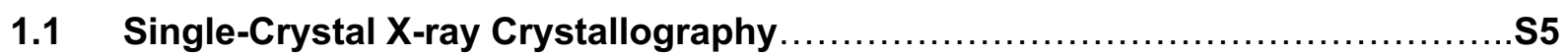

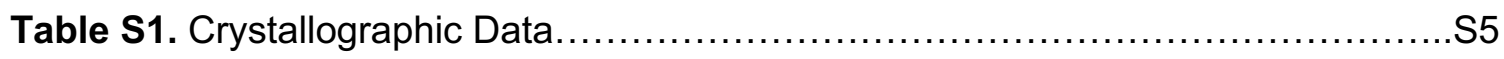

Table S2. Crystal data and structural refinement of

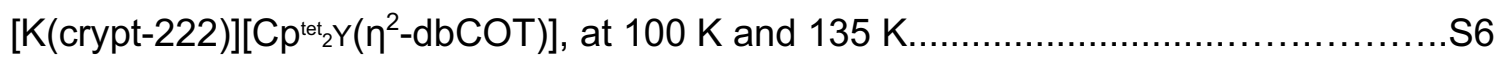

Table S3. Crystal data and structural refinement of

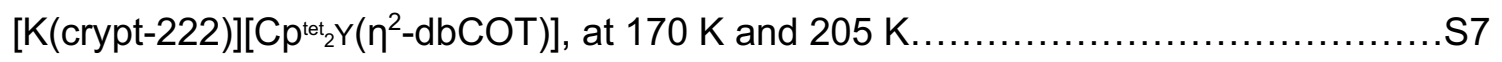

Table S4. Crystal data and structural refinement of

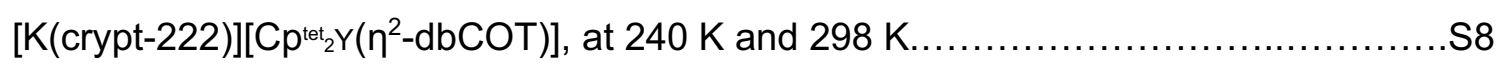

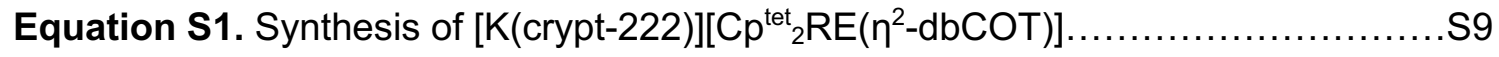

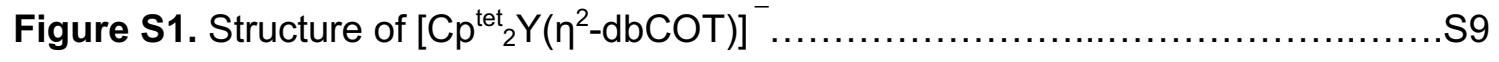

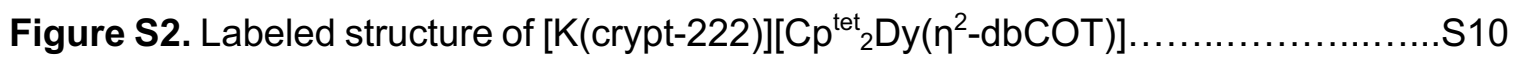

Figures S3 and S4. Structure of $\left[K(\right.$ crypt-222) $]\left[C p^{\text {tet }}{ }_{2} R E\left(\eta^{2}-d b C O T\right)\right] \ldots \ldots \ldots \ldots \ldots \ldots . . . . . \ldots 11$

Figures S5 and S6. Thermal ellipsoid plots of $[K(c r y p t-222)]\left[C p^{\text {tet }}{ }_{2} R E\left(\eta^{2}-d b C O T\right)\right] \ldots . . . S 12$

Figure S7. Space filling model of $[\mathrm{K}(\mathrm{crypt}-222)]\left[\mathrm{Cp}^{\text {tet }}{ }_{2} \mathrm{Y}\left(\mathrm{\eta}^{2}-\mathrm{dbCOT}\right)\right] \ldots \ldots \ldots \ldots \ldots \ldots . . . . \ldots \ldots$

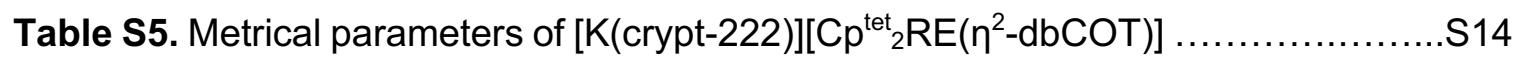

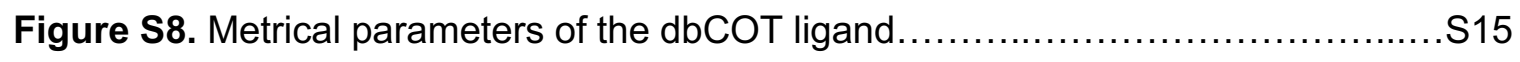

Figure S9. Overlay of VT structures of $[\mathrm{K}(\mathrm{crypt}-222)]\left[\mathrm{Cp}{ }^{\text {tet }}{ }_{2} \mathrm{Y}\left(\mathrm{\eta}^{2}-\mathrm{dbCOT}\right)\right] \ldots \ldots \ldots \ldots \ldots . \mathrm{S} 16$

Figure S10. Plot of shortest $\mathrm{Y}-\mathrm{C}_{\mathrm{dbcot}}$ distances versus temperature in

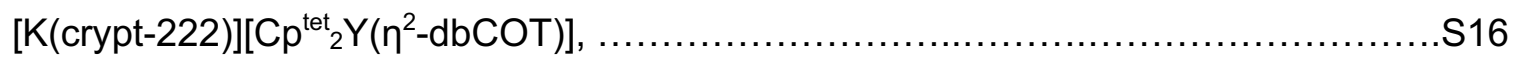

Table S6. Bond metrics of temperature-dependent single-crystal X-ray

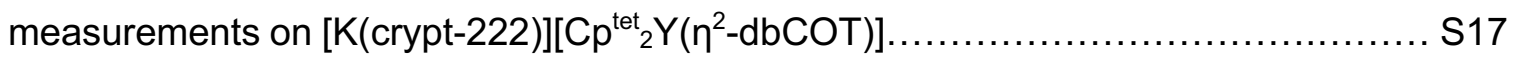

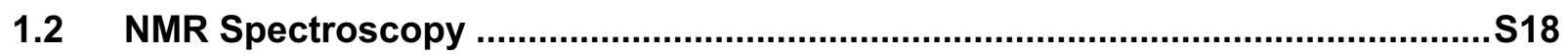

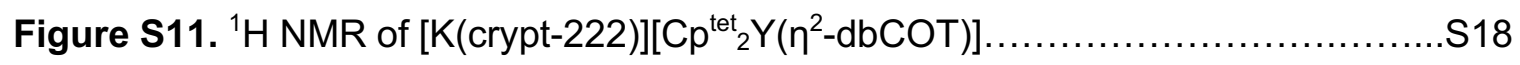

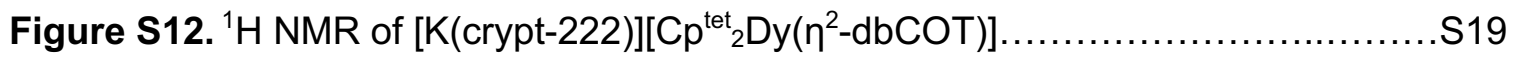

Figure S13. ${ }^{1} \mathrm{H}$ NMR of $\mathrm{K}_{2} \mathrm{dbCOT}(\mathrm{THF})_{1.5 \ldots \ldots \ldots \ldots \ldots \ldots \ldots \ldots \ldots \ldots \ldots \ldots \ldots \ldots \ldots \ldots \ldots \ldots \ldots \ldots \ldots \ldots \ldots \ldots \ldots} 20$

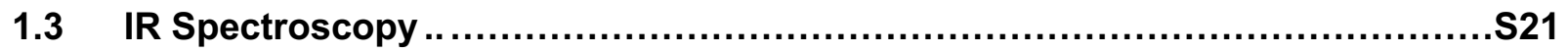

Figures S14 and S15. IR spectra of $\left[K\left(\right.\right.$ crypt-222)][Cp $\left.{ }_{2}^{\text {tet }} \mathrm{RE}\left(\eta^{2}-\mathrm{dbCOT}\right)\right] \ldots \ldots \ldots \ldots \ldots . . . .21$ 
Figures S16 and S17. Comparison of the experimental FTIR spectrum of

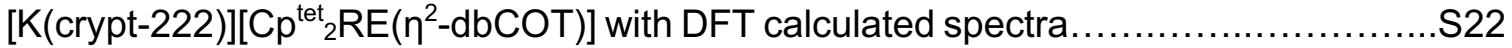

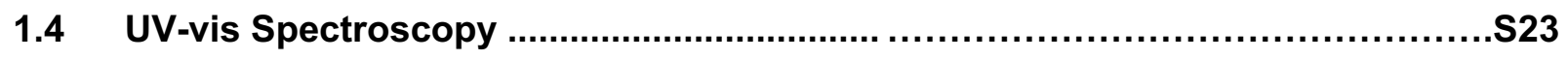

Figure S18. UV-vis spectra of $\left[K\left(\right.\right.$ crypt-222)][C $\left.\mathrm{p}^{\text {tet }}{ }_{2} \mathrm{Y}\left(\mathrm{n}^{2}-\mathrm{dbCOT}\right)\right] \ldots \ldots \ldots \ldots \ldots \ldots \ldots . \mathrm{S} 23$

Figure S19. Overlay of UV-vis spectrum of [K(crypt-222)][C $\left.{ }^{\text {tet }}{ }_{2} \mathrm{Y}\left(\mathrm{n}^{2}-\mathrm{dbCOT}\right)\right]$,

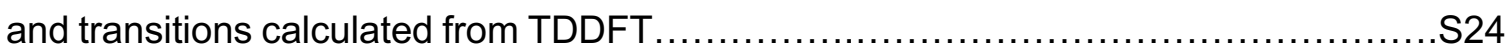

Figure S20. UV-vis spectra of $[K(\operatorname{crypt}-222)]\left[C p^{\text {tet }}{ }_{2} \mathrm{Dy}\left(\eta^{2}-\mathrm{dbCOT}\right)\right] \ldots \ldots \ldots \ldots \ldots \ldots \ldots . . \mathrm{S} 25$

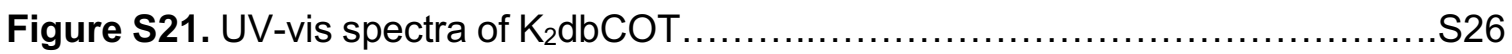

Figure S22. Overlay of UV-vis spectrum of $\mathrm{K}_{2} \mathrm{dbCOT}$, and transitions calculated from TDDFT

Figure S23. Overlay of UV-vis spectrum of [K(crypt-222)][C $\left.\mathrm{p}^{\text {tet }}{ }_{2} \mathrm{Y}\left(\eta^{2}-\mathrm{dbCOT}\right)\right]$, and transitions calculated from TDDFT for both 1 and $\mathrm{K}_{2} \mathrm{dbCOT}$ S28

\subsection{Electrochemistry} S29

Figure S24. Cyclic voltammograms of $[\mathrm{K}(\mathrm{crypt}-222)]\left[C \mathrm{p}^{\text {tet }}{ }_{2} \mathrm{Y}\left(\eta^{2}-\mathrm{dbCOT}\right)\right]$ S29

Figure S25. Cyclic voltammograms of [K(crypt-222)][C $\left.\mathrm{p}^{\text {tet }}{ }_{2} \mathrm{Dy}\left(\mathrm{n}^{2}-\mathrm{dbCOT}\right)\right]$. S30

Figure S26. Cyclic voltammograms of $\mathrm{K}_{2} \mathrm{dbCOT}$.

\subsection{Magnetic Data.}

Figure S27. In-phase $\left(\chi_{\mathrm{M}^{\prime}}\right)$ component of variable-temperature ac magnetic susceptibility data of $[\mathrm{K}(\mathrm{crypt}-222)]\left[\mathrm{Cp}{ }^{\text {tet }}{ }_{2} \mathrm{Dy}\left(\eta^{2}-\mathrm{dbCOT}\right)\right]$ und a 0 Oe applied dc field.

Figure S28. Cole-Cole plots for $[\mathrm{K}(\operatorname{crypt}-222)]\left[\mathrm{Cp}^{\text {tet }}{ }_{2} \mathrm{Dy}\left(\eta^{2}-\mathrm{dbCOT}\right)\right]$ under a 0 Oe applied dc field.

Figure S29. Fitted temperature dependence of the relaxation times collected under a 0 Oe applied dc field.

Figure S30. In-phase $\left(\chi_{\mathrm{M}^{\prime}}\right)$ component of variable-temperature ac magnetic susceptibility data of $\left[\mathrm{K}(\right.$ crypt-222) $]\left[\mathrm{Cp}^{\text {tet }}{ }_{2} \mathrm{Dy}\left(\mathrm{n}^{2}-\mathrm{dbCOT}\right)\right]$ und a $1000 \mathrm{Oe}$ applied dc field.

Figure S31. Cole-Cole plots for $[K(\operatorname{crypt}-222)]\left[C p^{\text {tet }}{ }_{2} D y\left(n^{2}-d b C O T\right)\right]$ under a 1000 Oe applied dc field. S34

Figure S32. Fitted temperature dependence of the relaxation times collected under a 
1000 Oe applied dc field

Figures S33. Field-dependent magnetization data for

$[\mathrm{K}(\mathrm{crypt}-222)]\left[\mathrm{C} \mathrm{p}^{\text {tet }}{ }_{2} \mathrm{Dy}\left(\mathrm{\eta}^{2} \mathrm{dbCOT}\right)\right]$.

Figures S34. Field-dependent magnetization loops for

$[\mathrm{K}(\mathrm{crypt}-222)]\left[\mathrm{Cp}^{\text {tet }}{ }_{2} \mathrm{Dy}\left(\mathrm{n}^{2}-\mathrm{dbCOT}\right)\right]$.

1.7 Calculated Orientation of the Magnetic Moment................................... 38

Table S7. Overview over different charge localization schemes........................ 38

Figure S35. Calculated orientation of the Dy magnetic moment...................... 39

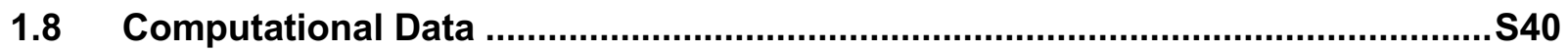

Table S8. Comparison of experimental and computed bond metrics B3LYP............S40

Table S9. Comparison of experimental and computed bond metrics PBE..............S41

Table S10. Comparison of experimental and computed bond metrics PBE0............S42

Table S11. Comparison of experimental and computed bond metrics TPSS .............S43

Table S12. Comparison of experimental and computed bond metrics TPSSh...........S44

Figure S36. Calculated Natural Bond Orbitals .................................... 45

Figure S37. Nucleus independent chemical shift values ........................... 46

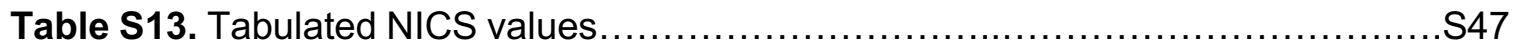

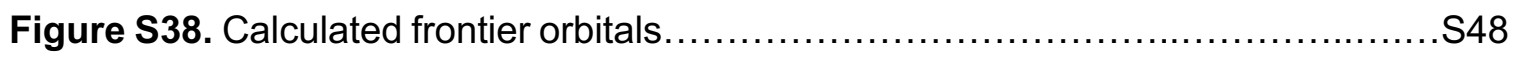

Table S14. Calculated transitions of $\left[\mathrm{Cp}^{\text {tet }}{ }_{2} \mathrm{Y}\left(\eta^{2}-\mathrm{dbCOT}\right)\right]^{-}$from time-dependent density functional theory calculations (TD-DFT).

Table S15. Calculated bond metrics from the optimized geometries of the temperature-dependent single-crystal $X$-ray measurements on

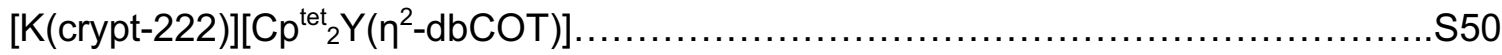

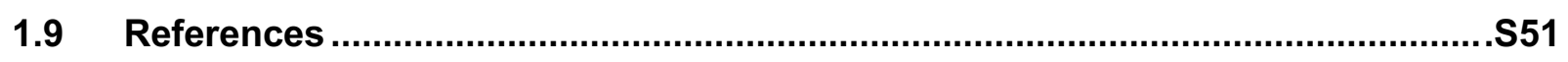




\subsection{Single-Crystal X-ray Crystallography}

Table S1. Crystal data and structural refinement of [K(crypt-222)][C $\left.p^{\mathrm{tet}}{ }_{2} R E\left(\eta^{2}-\mathrm{dbCOT}\right)\right],(R E=Y$ (1), Dy (2)).

\begin{tabular}{|c|c|c|}
\hline Compound & 1 & 2 \\
\hline Empirical formula & $\mathrm{C}_{52} \mathrm{H}_{74} \mathrm{KN}_{2} \mathrm{O}_{6} \mathrm{Y}$ & $\mathrm{C}_{52} \mathrm{H}_{73} \mathrm{DyKN}_{2} \mathrm{O}_{6}$ \\
\hline Formula weight $\left(\mathrm{g} \mathrm{mol}^{-1}\right)$ & 951.14 & 1023.72 \\
\hline Temperature (K) & $100.0(1)$ & $100.1(1)$ \\
\hline Crystal system & triclinic & triclinic \\
\hline Space group & $P-1$ & $P-1$ \\
\hline \multirow[t]{6}{*}{ Unit Cell Dimensions } & $a=10.7933(3) \AA$ & $a=10.8290(2) \AA$ \\
\hline & $b=13.9815(3) \AA$ & $b=13.9735(1) \AA$ \\
\hline & $c=17.6562(4) \AA$ & $c=17.6164(2) \AA$ \\
\hline & $\alpha=107.3103(19)^{\circ}$ & $\alpha=107.4650(1)^{\circ}$ \\
\hline & $B=90.2543(19)^{\circ}$ & $B=90.2610(1)^{\circ}$ \\
\hline & $y=100.4365(18)^{\circ}$ & $y=100 \cdot 4710(1)^{\circ}$ \\
\hline Volume $\left(\AA^{3}\right)$ & $2496.83(10)$ & $2495.50(6)$ \\
\hline$z$ & 2 & 2 \\
\hline$\rho_{\text {calc }}\left(\mathrm{g} \mathrm{cm}^{-3}\right)$ & 1.265 & 1.362 \\
\hline$\mu\left(\mathrm{mm}^{-1}\right)$ & 1.300 & 1.629 \\
\hline$F(000)$ & 1012.0 & 1064.0 \\
\hline Crystal size $\left(\mathrm{mm}^{3}\right)$ & $0.132 \times 0.094 \times 0.087$ & $0.458 \times 0.203 \times 0.171$ \\
\hline Radiation & $\operatorname{Mo~K}_{\alpha}(\lambda=0.71073)$ & $\operatorname{Mo~K}_{\alpha}(\lambda=0.71073)$ \\
\hline $2 \Theta$ range for data collection $\left({ }^{\circ}\right)$ & 4.414 to 62.012 & 4.406 to 58.094 \\
\hline Reflections collected & 53302 & 30040 \\
\hline Independent reflections & $12972 R_{\text {int }}=0.069$ & $11623 R_{\text {int }}=0.0418$ \\
\hline Data/restraints/parameters & $12972 / 0 / 578$ & $11623 / 0 / 567$ \\
\hline Goodness-of-fit on $F^{2}$ & 1.020 & 1.039 \\
\hline Final $R$ indexes $[/>=2 \sigma(I)]$ & $R_{1}=0.0482, \mathrm{w} R_{2}=0.0965$ & $R_{1}=0.0353, w R_{2}=0.0842$ \\
\hline Final $R$ indexes [all data] & $R_{1}=0.0714, \mathrm{w} R_{2}=0.1028$ & $R_{1}=0.0411, w R_{2}=0.0870$ \\
\hline Largest diff. peak/hole $\left(\mathrm{e} \AA^{-3}\right)$ & $1.70 /-0.84$ & $4.18 /-1.50$ \\
\hline
\end{tabular}


Table S2. Crystal data and structural refinement of $[\mathrm{K}(\mathrm{crypt}-222)]\left[\mathrm{C} \mathrm{p}^{\mathrm{tet}}{ }_{2} \mathrm{Y}\left(\eta^{2}-\mathrm{dbCOT}\right)\right]$, at $100 \mathrm{~K}$ $(1-100 \mathrm{~K})$ and $135 \mathrm{~K}(1-135 \mathrm{~K})$.

\begin{tabular}{|c|c|c|}
\hline Compound & 1-100 K & |1-135 K \\
\hline Empirical formula & $\mathrm{C}_{52} \mathrm{H}_{74} \mathrm{KN}_{2} \mathrm{O}_{6} \mathrm{Y}$ & $\mathrm{C}_{52} \mathrm{H}_{74} \mathrm{KN}_{2} \mathrm{O}_{6} \mathrm{Y}$ \\
\hline Formula weight $\left(\mathrm{g} \mathrm{mol}^{-1}\right)$ & 951.14 & 951.14 \\
\hline Temperature (K) & $100.0(1)$ & $135.1(5)$ \\
\hline Crystal system & triclinic & triclinic \\
\hline Space group & $P-1$ & $P-1$ \\
\hline Unit Cell Dimensions & $\begin{array}{l}\mathrm{a}=10.8061(2) \AA \\
\mathrm{b}=13.9650(2) \AA \\
\mathrm{c}=17.6427(3) \AA \\
\mathrm{a}=107.424(1)^{\circ} \\
\mathrm{B}=90.179(1)^{\circ} \\
\mathrm{y}=100.377(1)^{\circ}\end{array}$ & $\begin{array}{l}a=10.8076(2) \AA \\
b=13.9922(2) \AA \\
c=17.6871(3) \AA \\
\alpha=107.364(2)^{\circ} \\
B=90.162(1)^{\circ} \\
y=100.409(1)^{\circ}\end{array}$ \\
\hline Volume $\left(\AA^{3}\right)$ & $2494.07(7)$ & $2506.19(8)$ \\
\hline$Z$ & 2 & 2 \\
\hline$\rho_{\text {calc }}\left(\mathrm{g} \mathrm{cm}^{-3}\right)$ & 1.267 & 1.280 \\
\hline$\mu\left(\mathrm{mm}^{-1}\right)$ & 1.301 & 1.372 \\
\hline$F(000)$ & 1012.0 & 1026.0 \\
\hline Crystal size $\left(\mathrm{mm}^{3}\right)$ & $0.3 \times 0.25 \times 0.23$ & $0.49 \times 0.24 \times 0.2$ \\
\hline Radiation & $\operatorname{Mo~K}_{\alpha}(\lambda=0.71073)$ & $\operatorname{Mo~K}_{\alpha}(\lambda=0.71073)$ \\
\hline $2 \Theta$ range for data collection $\left(^{\circ}\right)$ & 4.414 to 61.766 & 4.412 to 61.916 \\
\hline Reflections collected & 52355 & 52703 \\
\hline Independent reflections & $12840 R_{\mathrm{int}}=0.0356$ & $12876 R_{\mathrm{int}}=0.038$ \\
\hline Data/restraints/parameters & $12840 / 0 / 669$ & $12876 / 0 / 595$ \\
\hline Goodness-of-fit on $F^{2}$ & 1.028 & 1.017 \\
\hline Final $R$ indexes $[I>=2 \sigma(I)]$ & $R_{1}=0.0361, w R_{2}=0.0727$ & $R_{1}=0.0386, w R_{2}=0.0772$ \\
\hline Final $R$ indexes [all data] & $R_{1}=0.0450, \mathrm{w} R_{2}=0.0748$ & $R_{1}=0.0496, w R_{2}=0.0800$ \\
\hline Largest diff. peak/hole $\left(\mathrm{e} \AA^{-3}\right)$ & $0.86 /-0.51$ & $0.87 /-0.53$ \\
\hline
\end{tabular}


Table S3. Crystal data and structural refinement of $[\mathrm{K}(\operatorname{crypt}-222)]\left[\mathrm{Cp}^{\mathrm{tet}}{ }_{2} \mathrm{Y}\left(\eta^{2}-\mathrm{dbCOT}\right)\right]$, at $170 \mathrm{~K}$ (1-170 K) and $205 \mathrm{~K}(1-205 \mathrm{~K})$.

\begin{tabular}{|c|c|c|}
\hline Compound & $1-170 \mathrm{~K}$ & $1-205 \mathrm{~K}$ \\
\hline Empirical formula & $\mathrm{C}_{52} \mathrm{H}_{74} \mathrm{KN}_{2} \mathrm{O}_{6} \mathrm{Y}$ & $\mathrm{C}_{52} \mathrm{H}_{74} \mathrm{KN}_{2} \mathrm{O}_{6} \mathrm{Y}$ \\
\hline Formula weight $\left(\mathrm{g} \mathrm{mol}^{-1}\right)$ & 951.14 & 951.14 \\
\hline Temperature (K) & $170.0(1)$ & $205.0(1)$ \\
\hline Crystal system & triclinic & triclinic \\
\hline Space group & $P-1$ & $P-1$ \\
\hline Unit Cell Dimensions & $\begin{array}{l}a=10.8015(2) \AA \\
b=14.0189(3) \AA \\
c=17.7294(4) \AA \\
a=107.266(2)^{\circ} \\
B=90.147(2)^{\circ} \\
y=100.425(2)^{\circ}\end{array}$ & $\begin{array}{l}a=10.7980(3) \AA \\
b=14.0553(3) \AA \\
c=17.7766(4) \AA \\
a=107.154(2)^{\circ} \\
B=90.176(2)^{\circ} \\
y=100.436(2)^{\circ}\end{array}$ \\
\hline Volume $\left(\AA^{3}\right)$ & 2516.85(11) & $2530.69(11)$ \\
\hline$Z$ & 2 & 2 \\
\hline$\rho_{\text {calc }}\left(\mathrm{g} \mathrm{cm}^{-3}\right)$ & 1.255 & 1.248 \\
\hline$\mu\left(\mathrm{mm}^{-1}\right)$ & 1.290 & 1.283 \\
\hline$F(000)$ & 1012.0 & 1012.0 \\
\hline Crystal size $\left(\mathrm{mm}^{3}\right)$ & $0.377 \times 0.226 \times 0.205$ & $0.251 \times 0.243 \times 0.197$ \\
\hline Radiation & Mo $\mathrm{K}_{\alpha}(\lambda=0.71073)$ & Mo $\mathrm{K}_{\alpha}(\lambda=0.71073)$ \\
\hline $2 \Theta$ range for data collection $\left({ }^{\circ}\right)$ & 4.41 to 61.908 & 4.408 to 61.948 \\
\hline Reflections collected & 52792 & 53247 \\
\hline Independent reflections & $12923 R_{\text {int }}=0.0502$ & $13004 R_{\text {int }}=0.0502$ \\
\hline Data/restraints/parameters & $12923 / 0 / 583$ & $13004 / 0 / 579$ \\
\hline Goodness-of-fit on $F^{2}$ & 1.012 & 1.027 \\
\hline Final $R$ indexes $[I>=2 \sigma(I)]$ & $R_{1}=0.0432, w R_{2}=0.0876$ & $R_{1}=0.0450, \mathrm{w} R_{2}=0.0861$ \\
\hline Final $R$ indexes [all data] & $R_{1}=0.0574, w R_{2}=0.0916$ & $R_{1}=0.0642, w R_{2}=0.0911$ \\
\hline Largest diff. peak/hole $\left(\mathrm{e} \AA^{-3}\right)$ & $0.58 /-0.66$ & $0.72 /-0.54$ \\
\hline
\end{tabular}


Table S4. Crystal data and structural refinement of [K(crypt-222)][C $\left.\mathrm{p}^{\operatorname{tet}_{2}} \mathrm{Y}\left(\eta^{2}-\mathrm{dbCOT}\right)\right]$, at $240 \mathrm{~K}$ (1$240 \mathrm{~K})$ and $298 \mathrm{~K}(1-298 \mathrm{~K})$.

Compound

Empirical formula

Formula weight $\left(\mathrm{g} \mathrm{mol}^{-1}\right)$

Temperature (K)

Crystal system

Space group

Unit Cell Dimensions

Volume $\left(\AA^{3}\right)$

$Z$

$\rho_{\text {calc }}\left(\mathrm{g} \mathrm{cm}^{-3}\right)$

$\mu\left(\mathrm{mm}^{-1}\right)$

F(000)

Crystal size $\left(\mathrm{mm}^{3}\right)$

Radiation

$2 \Theta$ range for data collection $\left({ }^{\circ}\right)$

Reflections collected

Independent reflections

Data/restraints/parameters

Goodness-of-fit on $F^{2}$

Final $R$ indexes $[/>=2 \sigma(I)]$

Final $R$ indexes [all data]

Largest diff. peak/hole (e $\AA^{-3}$ )

$\mid$\begin{tabular}{l|l}
$1-240 ~ K$ \\
$\mathrm{C}_{52} \mathrm{H}_{74} \mathrm{KN}_{2} \mathrm{O}_{6} \mathrm{Y}$
\end{tabular}

951.14

240.0(1)

triclinic

$P-1$

$a=10.7977(3) \AA$

$\mathrm{b}=14.0930(4) \AA$

$c=17.8185(5) \AA$

$\alpha=107.051(2)^{\circ}$

$B=90.194(2)^{\circ}$

$y=100.468(2)^{\circ}$

2544.51(13)

2

1.241

1.276

1012.0

$0.355 \times 0.248 \times 0.195$

$\operatorname{Mo~K}_{\alpha}(\lambda=0.71073)$

4.404 to 61.74

53392

$13065 R_{\text {int }}=0.0474$

$13065 / 0 / 641$

1.020

$R_{1}=0.0500, w R_{2}=0.0952$

$R_{1}=0.0757, \mathrm{w} R_{2}=0.1022$

$0.59 /-0.51$

\section{1-298 K}

$\mathrm{C}_{52} \mathrm{H}_{74} \mathrm{KN}_{2} \mathrm{O}_{6} \mathrm{Y}$

951.14

297.9(1)

triclinic

$P-1$

$a=10.7936(3) \AA$

$\mathrm{b}=14.1453(4) \AA$

$c=17.8670(4) \AA$

$\alpha=106.962(2)^{\circ}$

$\mathrm{B}=90.238(2)^{\circ}$

$y=100.569(3)^{\circ}$

2560.15(12)

2

1.234

1.268

1012.0

$0.283 \times 0.217 \times 0.176$

Mo $\mathrm{K}_{\alpha}(\lambda=0.71073)$

4.4 to 61.552

53619

$13128 R_{\text {int }}=0.0564$

$13128 / 0 / 567$

1.033

$R_{1}=0.0585, w R_{2}=0.1077$

$R_{1}=0.0982, \mathrm{w} R_{2}=0.1187$

$0.55 /-0.44$ 

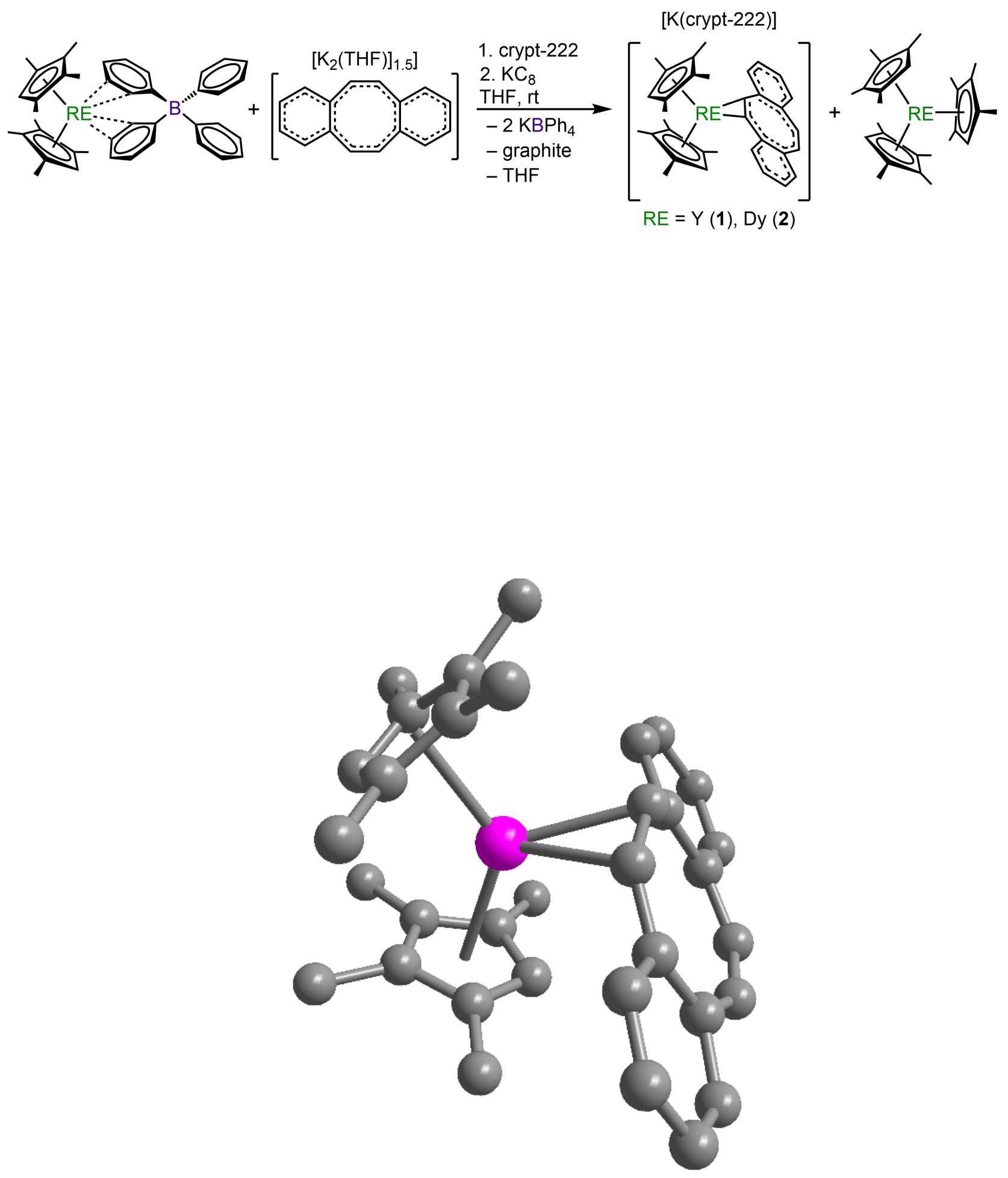

Figure S1. Structure of the $\left[\mathrm{Cp}{ }^{\text {tet }} \mathrm{Y} Y\left(\mathrm{n}^{2}-\mathrm{dbCOT}\right)\right]^{-}$anion in a crystal of $[\mathrm{K}(\mathrm{crypt}-222)]\left[\mathrm{Cp}{ }^{\text {tet }}{ }_{2} \mathrm{Y}\left(\mathrm{n}^{2}-\right.\right.$ $\mathrm{dbCOT})]$ (1). Pink and gray spheres represent $Y$ and $C$ atoms, respectively. Hydrogen atoms and the $\left[\mathrm{K}(\text { crypt-222) }]^{+}\right.$countercation have been removed for clarity. 


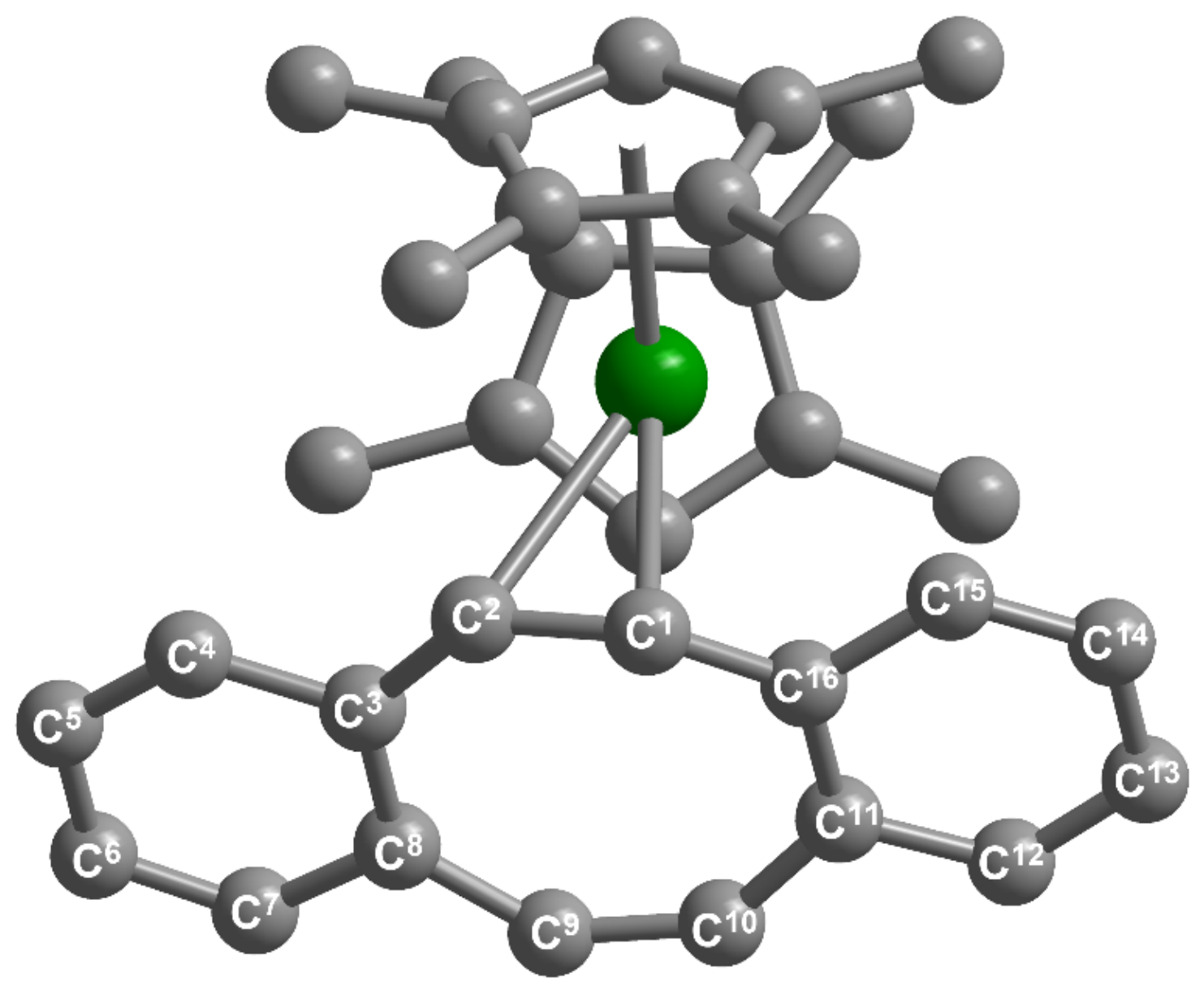

Figure S2. Structure of the $\left[C p^{\text {tet }}{ }_{2} \mathrm{Dy}\left(\eta^{2}-\mathrm{dbCOT}\right)\right]^{-}$anion in a crystal of $[\mathrm{K}(\mathrm{crypt}-222)]\left[\mathrm{Cp}{ }^{\text {tet }}{ }_{2} \mathrm{Dy}\left(\eta^{2}-\right.\right.$ $\mathrm{dbCOT})](2)$. Green and gray spheres represent Dy and C atoms, respectively. Hydrogen atoms and the $\left[\mathrm{K}(\text { crypt-222) }]^{+}\right.$countercation have been removed for clarity. Labeling scheme for the carbon atoms of the $(\mathrm{dbCOT})^{2-}$ anion. 


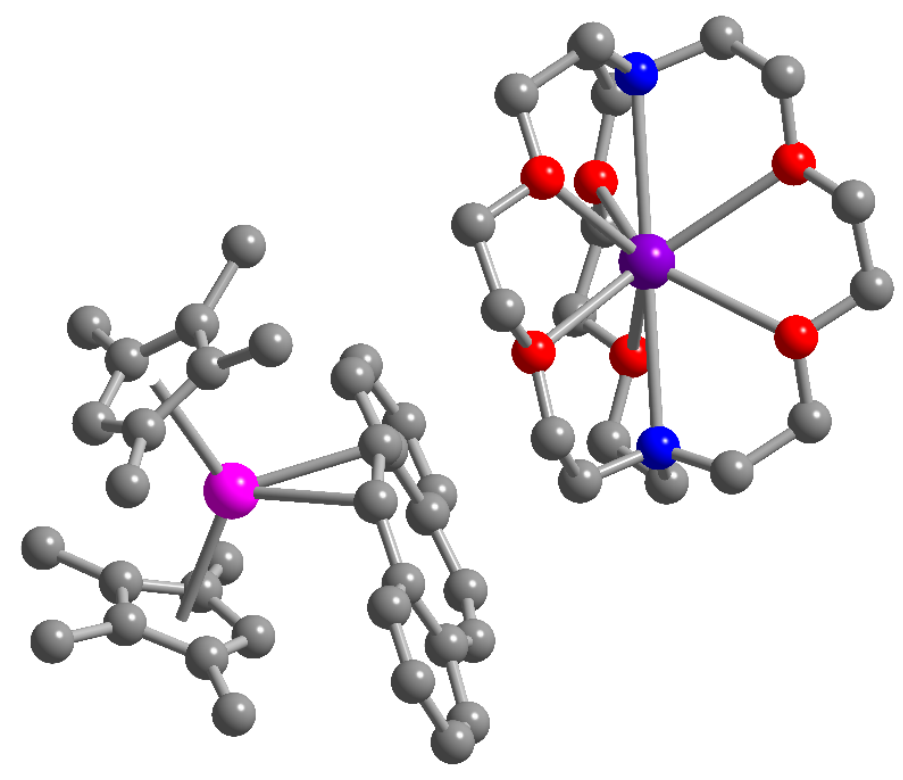

Figure S3. Structure of $\left[K(\right.$ crypt-222) $]\left[C p^{\text {tet }}{ }_{2} Y\left(\eta^{2}-d b C O T\right)\right](1)$. Pink, purple, blue, red, and gray spheres represent $\mathrm{Y}, \mathrm{K}, \mathrm{N}, \mathrm{O}$, and $\mathrm{C}$ atoms, respectively. $\mathrm{H}$ atoms have been omitted for clarity.

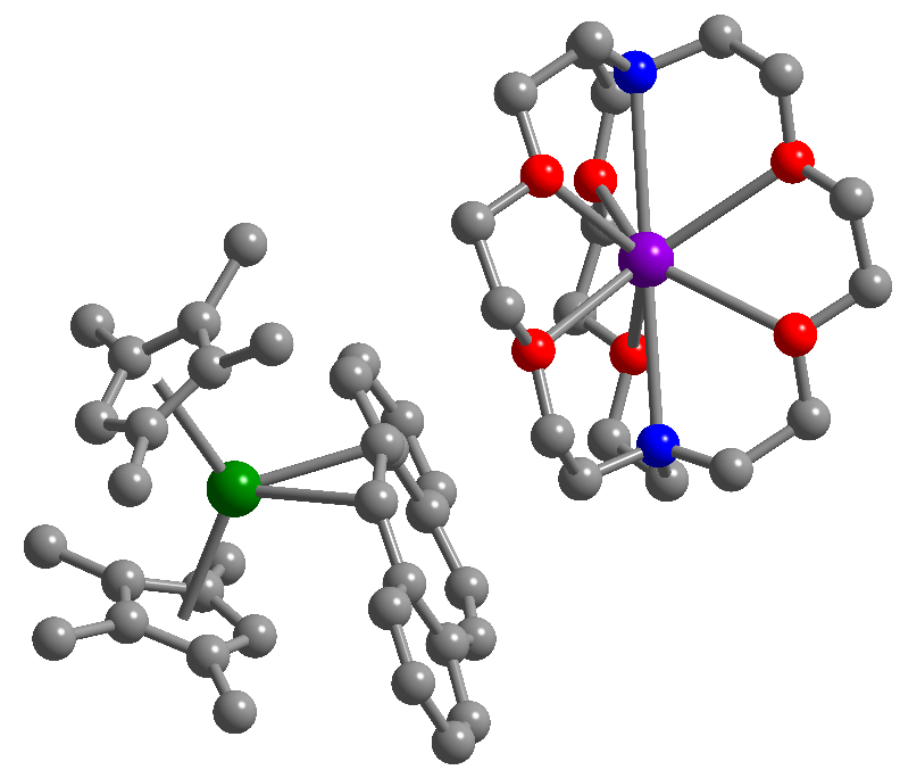

Figure S4. Structure of [K(crypt-222)][C $\left.{ }^{\text {tet }}{ }_{2} \mathrm{Dy}\left(\eta^{2}-\mathrm{dbCOT}\right)\right]$ (2). Green, purple, blue, red, and gray spheres represent Dy, K, N, O, and C atoms, respectively. $\mathrm{H}$ atoms have been omitted for clarity. 


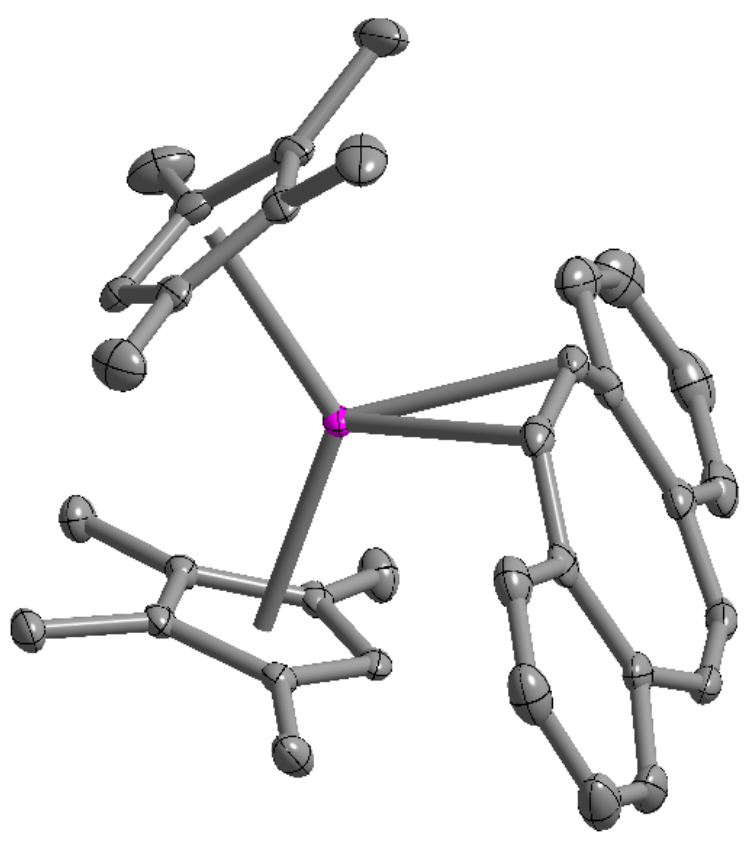

Figure S5. Structure of the $\left[\mathrm{Cp}^{\text {tet }}{ }_{2} \mathrm{Y}\left(\eta^{2}-\mathrm{dbCOT}\right)\right]^{-}$anion in a crystal of [K(crypt-222)][C $\mathrm{p}^{\text {tet }}{ }_{2} \mathrm{Y}\left(\mathrm{n}^{2}-\right.$ $\mathrm{dbCOT})$ ] (1), with thermal ellipsoids drawn at $50 \%$ probability level. Pink and gray spheres represent $\mathrm{Y}$ and $\mathrm{C}$ atoms, respectively. Hydrogen atoms and the $[\mathrm{K}(\mathrm{crypt}-222)]^{+}$countercation have been removed for clarity.

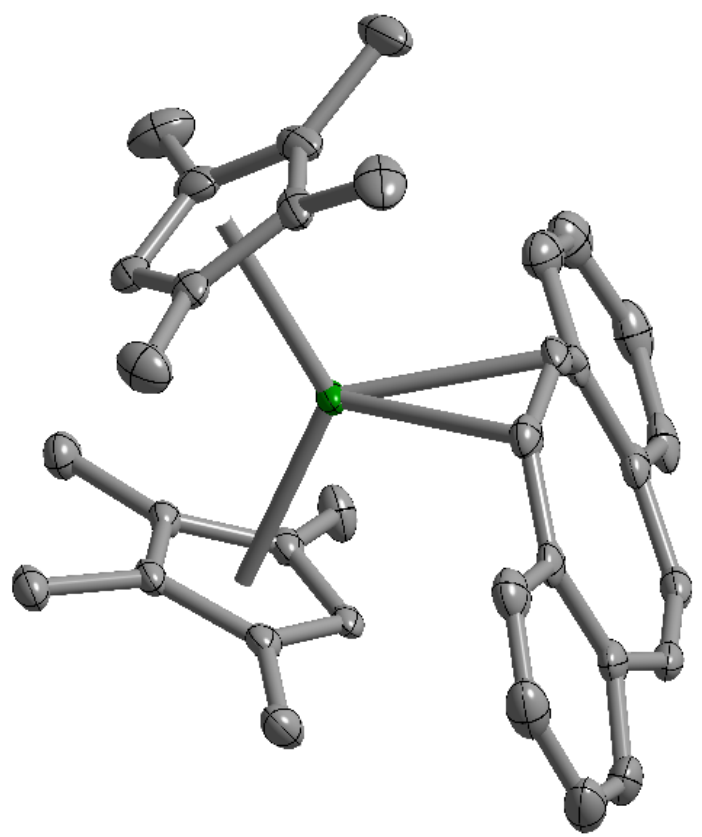

Figure S6. Structure of the $\left[\mathrm{Cp}^{\text {tet }}{ }_{2} \mathrm{Dy}\left(\eta^{2}-\mathrm{dbCOT}\right)\right]^{-}$anion in a crystal of [K(crypt-222)][Cp ${ }^{\text {tet }}{ }_{2} \mathrm{Dy}\left(\eta^{2}-\right.$ $\mathrm{dbCOT})$ ] (2), with thermal ellipsoids drawn at 50\% probability level. Green and gray spheres represent Dy and $\mathrm{C}$ atoms, respectively. Hydrogen atoms and the [K(crypt-222)] ${ }^{+}$counter cation have been removed for clarity. 


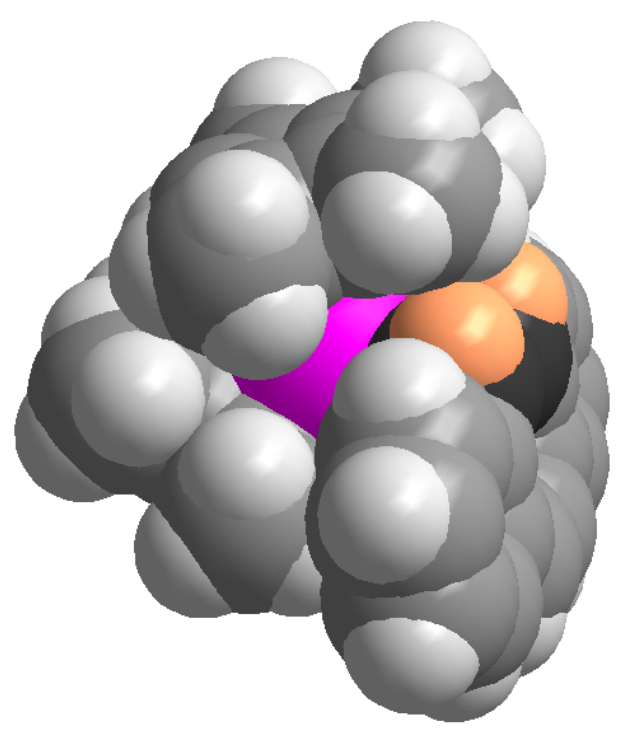

Figure S7. Space filling model of the $\left[\mathrm{Cp}^{\text {tet }}{ }_{2} \mathrm{Y}\left(\eta^{2}-\mathrm{dbCOT}\right)\right]^{-}$anion in a crystal of [K(crypt$222)]\left[C p^{\text {tet }}{ }_{2} Y\left(\eta^{2}-d b C O T\right)\right](1)$. Pink, gray, and white-gray spheres represent $\mathrm{Y}, \mathrm{C}$, and $\mathrm{H}$ atoms, respectively. The $\left[\mathrm{K}(\text { crypt-222) }]^{+}\right.$countercation has been removed for clarity. Selected $\mathrm{C}$ and $\mathrm{H}$ atoms have been highlighted in dark gray and orange, respectively, to show the deviation of planarity for select $\mathrm{C}$ atoms and their corresponding $\mathrm{H}$ atoms. 
Table S5. Metrical parameters from the crystal structures of $[K(\operatorname{crypt}-222)]\left[C p^{\text {tet }}{ }_{2} R E\left(\eta^{2}-d b C O T\right)\right]$ $(R E=Y, 1$ and Dy, 2). Distances are given in angstroms $(\AA)$ and angles are given in degrees (deg). Atom labels correspond to those shown in Figure S2.

\begin{tabular}{|c|c|c|c|}
\hline & & {$\left[\left(\mathrm{Cp}^{\text {tet }}{ }_{2}\right) \mathrm{Y}\left(\mathrm{n}^{2}-\mathrm{dbCOT}\right)\right]$} & {$\left[\left(\mathrm{Cp}^{\mathrm{tet}}{ }_{2}\right) \mathrm{Dy}\left(\mathrm{n}^{2}-\mathrm{dbCOT}\right)\right]$} \\
\hline \multicolumn{4}{|c|}{ Distances $(\AA)$} \\
\hline \multirow[t]{5}{*}{$\mathrm{RE}-\mathrm{C}\left(\mathrm{Cp}^{\mathrm{tet}}{ }_{1}\right)$} & $\mathrm{C}_{45}$ & $2.754(2)$ & $2.768(2)$ \\
\hline & $\mathrm{C}_{46}$ & $2.774(2)$ & $2.790(3)$ \\
\hline & $\mathrm{C}_{47}$ & $2.664(2)$ & $2.676(4)$ \\
\hline & $\mathrm{C}_{48}$ & $2.580(2)$ & $2.582(3)$ \\
\hline & $\mathrm{C}_{49}$ & $2.644(2)$ & $2.653(3)$ \\
\hline $\mathrm{RE}-\mathrm{C} \mathrm{p}_{\text {cent }}^{\text {tet }}$ & & $2.400(1)$ & $2.411(1)$ \\
\hline \multirow[t]{5}{*}{$\mathrm{RE}-\mathrm{C}\left(\mathrm{Cp}^{\mathrm{tet}}{ }_{2}\right)$} & $\mathrm{C}_{54}$ & $2.603(2)$ & $2.613(3)$ \\
\hline & $\mathrm{C}_{55}$ & $2.615(2)$ & $2.616(2)$ \\
\hline & $\mathrm{C}_{56}$ & $2.677(2)$ & $2.686(3)$ \\
\hline & $\mathrm{C}_{57}$ & $2.718(2)$ & $2.729(3)$ \\
\hline & $\mathrm{C}_{58}$ & $2.683(2)$ & $2.694(3)$ \\
\hline $\mathrm{RE}-\mathrm{C} \mathrm{p}^{\text {tet }}{ }_{\text {cent }}$ & & $2.370(1)$ & $2.381(1)$ \\
\hline$C p^{\text {tet }}{ }_{\text {cent }}-\mathrm{RE}-\mathrm{C} \mathrm{p}^{\text {tet }}{ }_{\text {cent }}$ & & $128.67(4)$ & $128.03(1)$ \\
\hline \multirow[t]{4}{*}{$\mathrm{RE}-\mathrm{C}_{\mathrm{dbCOT}}$} & $\mathrm{C}_{1}$ & $2.467(2)$ & $2.463(3)$ \\
\hline & $\mathrm{C}_{2}$ & $2.567(2)$ & $2.573(3)$ \\
\hline & $\mathrm{C}_{3}$ & $2.897(3)$ & $2.833(4)$ \\
\hline & $\mathrm{C}_{16}$ & $3.444(3)$ & $3.462(3)$ \\
\hline
\end{tabular}



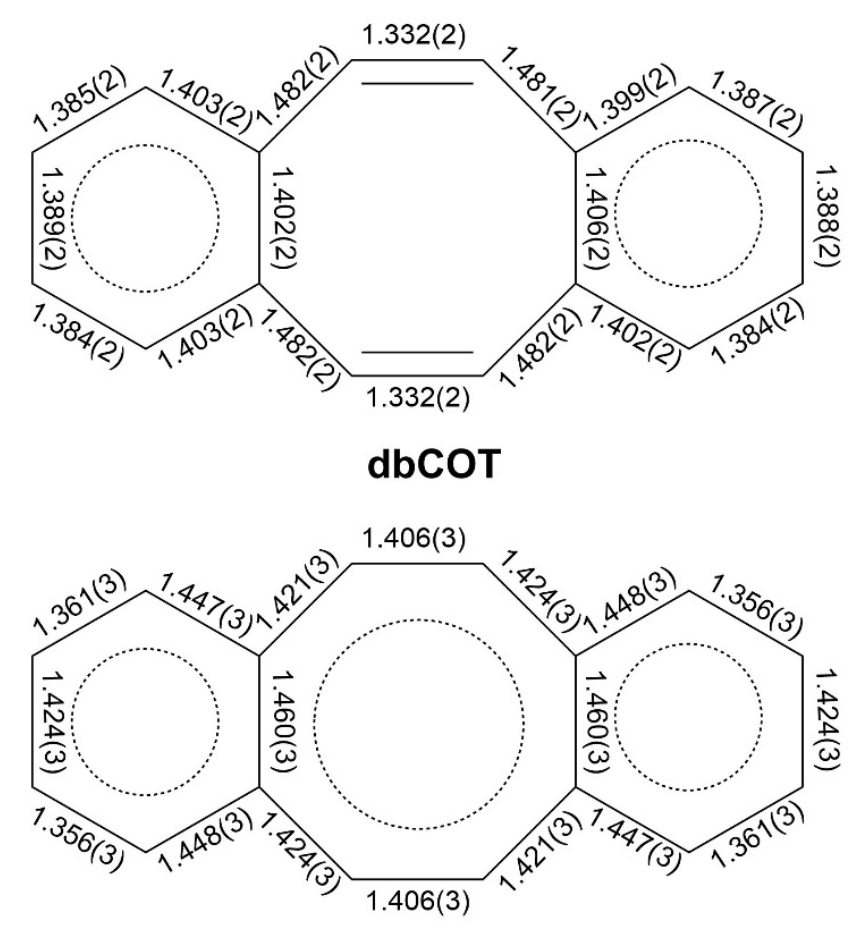

$(\mathrm{dbCOT})^{2-}$ in $\mathrm{K}_{2}$ [dbCOT]

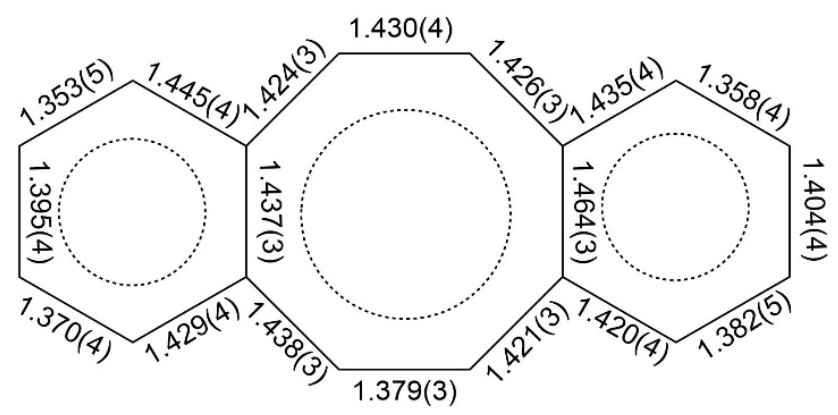

$(\mathrm{dbCOT})^{2-}$ in [Cp $\left.{ }_{2}^{\text {tet }}{ }_{2} \mathrm{Y}\left(\eta^{2}-\mathrm{dbCOT}\right)\right]^{-}$

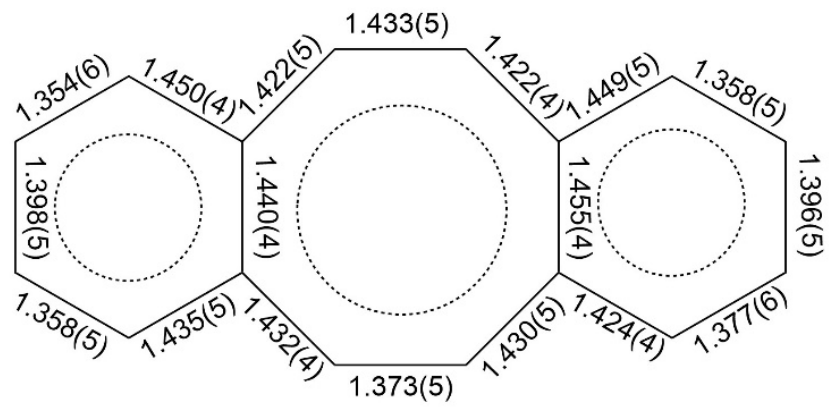

\section{$(\mathrm{dbCOT})^{2-}$ in $\left[\mathrm{Cp}{ }^{\text {tet }}{ }_{2} \mathrm{Dy}\left(\eta^{2}-\mathrm{dbCOT}\right)\right]^{-}$}

Figure S8. Metrical data of dbCOT from the crystal structures of dbCOT, ${ }^{1} \mathrm{~K}_{2}[\mathrm{dbCOT}],{ }^{1}\left[\mathrm{Cp}^{\mathrm{tet}}{ }_{2} \mathrm{Y}\left(\mathrm{\eta}^{2}-\right.\right.$ $\mathrm{dbCOT})]^{-}$, and $\left[\mathrm{Cp}^{\text {tet }}{ }_{2} \mathrm{Dy}\left(\eta^{2}-\mathrm{dbCOT}\right)\right]$. Distances are given in angstroms $(\AA)$. 


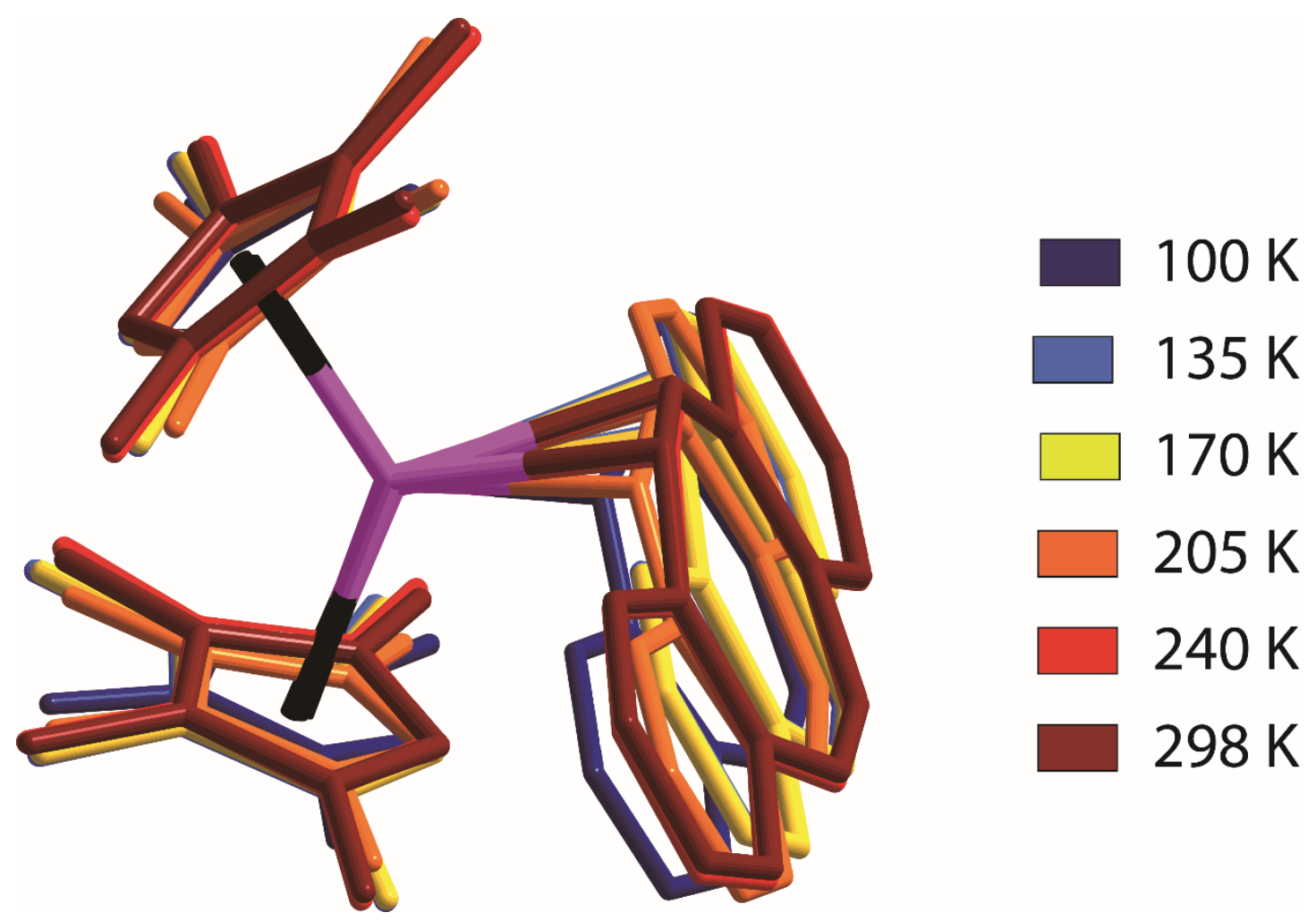

Figure S9. Overlay of structures of $\left.\mathrm{Cp}^{\text {tet }}{ }_{2} \mathrm{Y}\left(\eta^{2}-\mathrm{dbCOT}\right)\right]^{-}(1)$, collected at temperatures between $100 \mathrm{~K}$ and $298 \mathrm{~K}$ via single-crystal $\mathrm{X}$-ray diffraction. Crystal data and structural refinement parameters are given in Tables S2-S4.

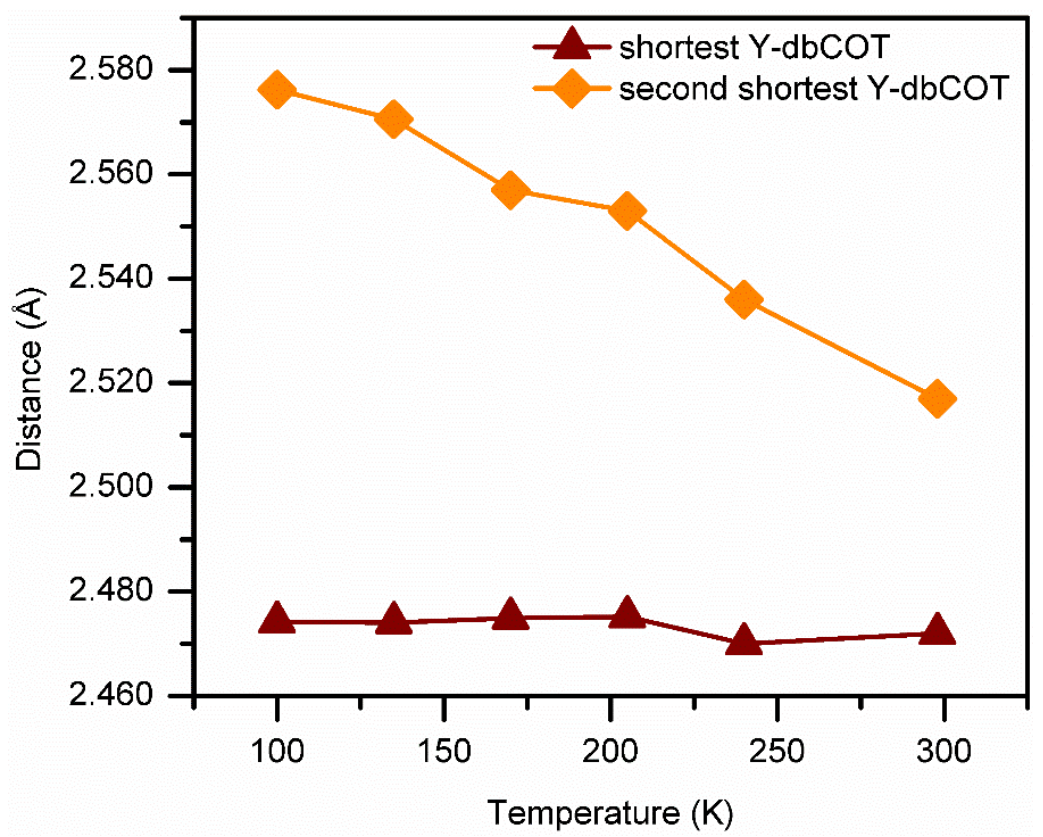

Figure S10. Plot of shortest $\mathrm{Y}-\mathrm{C}_{\mathrm{dbcot}}$ distance versus temperature in $[\mathrm{K}(\mathrm{crypt}-222)]\left[\mathrm{Cp}{ }^{\text {tet }}{ }_{2} \mathrm{Y}\left(\eta^{2}-\right.\right.$ dbCOT)], (1). 
Table S6. Bond metrics of temperature-dependent single-crystal $X$-ray diffraction measurements on [K(crypt-222)][Cp $\left.{ }_{2}^{\text {tet }} \mathrm{Y}\left(\mathrm{n}^{2}-\mathrm{dbCOT}\right)\right](1)$.

\begin{tabular}{|c|c|c|c|c|c|c|}
\hline Atoms & $100 \mathrm{~K}^{*}$ & $135 \mathrm{~K}$ & $170 \mathrm{~K}$ & $205 \mathrm{~K}$ & $240 \mathrm{~K}$ & $298 \mathrm{~K}$ \\
\hline & \multicolumn{6}{|c|}{ Y-dbCOT distances $(\AA)$} \\
\hline 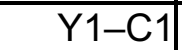 & $2.474(2)$ & $2.571(2)$ & $2.557(2)$ & $2.475(2)$ & $2.536(2)$ & $2.517(3)$ \\
\hline $\mathrm{Y} 1-\mathrm{C} 2$ & $2.576(2)$ & $2.474(2)$ & $2.475(2)$ & $2.553(2)$ & $2.470(2)$ & $2.472(3)$ \\
\hline Y1-C3 & $3.452(1)$ & $2.914(2)$ & $2.945(2)$ & $3.390(3)$ & $3.010(3)$ & $3.067(4)$ \\
\hline \multirow[t]{2}{*}{$\mathrm{Y} 1-\mathrm{C} 16$} & $2.891(2)$ & $3.438(2)$ & $3.418(3)$ & $2.976(28)$ & $3.361(33)$ & $3.321(1)$ \\
\hline & \multicolumn{6}{|c|}{$Y-C p^{\text {tet }}$ distances (top) $(\AA)$} \\
\hline Y1-C26 & $2.605(2)$ & $2.603(2)$ & $2.601(2)$ & $2.600(2)$ & $2.597(2)$ & $2.597(2)$ \\
\hline $\mathrm{Y} 1-\mathrm{C} 27$ & $2.615(2)$ & $2.683(2)$ & $2.678(2)$ & $2.618(2)$ & $2.675(2)$ & $2.668(3)$ \\
\hline $\mathrm{Y} 1-\mathrm{C} 28$ & $2.680(2)$ & $2.719(2)$ & $2.717(2)$ & $2.686(2)$ & $2.720(2)$ & $2.717(2)$ \\
\hline Y1-C29 & $2.719(2)$ & $2.680(2)$ & $2.685(2)$ & $2.718(2)$ & $2.689(2)$ & $2.690(2)$ \\
\hline \multirow[t]{2}{*}{$\mathrm{Y} 1-\mathrm{C} 30$} & $2.683(2)$ & $2.616(2)$ & $2.615(2)$ & $2.676(2)$ & $2.621(2)$ & $2.619(2)$ \\
\hline & \multicolumn{6}{|c|}{ Y-Cp $p^{\text {tet }}$ distances (bottom) $(\AA)$} \\
\hline $\mathrm{Y} 1-\mathrm{C} 17$ & $2.755(2)$ & $2.774(2)$ & $2.774(2)$ & $2.757(2)$ & $2.778(2)$ & $2.776(3)$ \\
\hline Y1-C18 & $2.775(2)$ & $2.757(2)$ & $2.757(2)$ & $2.775(2$ & $2.764(2)$ & $2.761(3)$ \\
\hline Y1-C19 & $2.666(2)$ & $2.642(2)$ & $2.641(2)$ & $2.661(2)$ & $2.641(2)$ & $2.641(3)$ \\
\hline Y1-C20 & $2.578(2)$ & $2.577(2)$ & $2.576(2)$ & $2.576(2)$ & $2.574(2)$ & $2.572(3)$ \\
\hline \multirow[t]{2}{*}{$\mathrm{Y} 1-\mathrm{C} 21$} & $2.642(2)$ & $2.665(2)$ & $2.662(2)$ & $2.642(2)$ & $2.663(3)$ & $2.660(3)$ \\
\hline & \multicolumn{6}{|c|}{ Distances around dbCOT rings $(\AA)$} \\
\hline $\mathrm{C} 1-\mathrm{C} 2$ & $1.433(2)$ & $1.429(3)$ & $1.425(3)$ & $1.423(4)$ & $1.419(4)$ & $1.417(5)$ \\
\hline C2-C3 & $1.424(2)$ & $1.428(3)$ & $1.430(3)$ & $1.405(4)$ & $1.441(4)$ & $1.453(5)$ \\
\hline C3-C4 & $1.442(2)$ & $1.438(3)$ & $1.437(3)$ & $1.458(4)$ & $1.422(5)$ & $1.422(6)$ \\
\hline C4-C5 & $1.360(1)$ & $1.354(3)$ & $1.345(4)$ & $1.351(4)$ & $1.326(6)$ & $1.321(7)$ \\
\hline C6-C5 & $1.404(3)$ & $1.404(4)$ & $1.400(4)$ & $1.383(5)$ & $1.401(6)$ & $1.392(9)$ \\
\hline C7-C6 & $1.364(3)$ & $1.371(3)$ & $1.380(4)$ & $1.355(4)$ & $1.400(5)$ & $1.403(7)$ \\
\hline C8-C7 & $1.420(3)$ & $1.420(3)$ & $1.420(3)$ & $1.430(4)$ & $1.413(4)$ & $1.417(5)$ \\
\hline C8-C9 & $1.434(2)$ & $1.426(3)$ & $1.427(3)$ & $1.432(3)$ & $1.426(4)$ & $1.418(5)$ \\
\hline C9-C10 & $1.381(3)$ & $1.377(3)$ & $1.377(3)$ & $1.371(3)$ & $1.367(4)$ & $1.366(5)$ \\
\hline C10-C11 & $1.424(2)$ & $1.428(3)$ & $1.431(3)$ & $1.427(3)$ & $1.435(4)$ & $1.424(5)$ \\
\hline C11-C12 & $1.424(2)$ & $1.428(3)$ & $1.431(3)$ & $1.416(4)$ & $1.435(4)$ & $1.430(5)$ \\
\hline C12-C13 & $1.370(3)$ & $1.364(3)$ & $1.363(4)$ & $1.389(5)$ & $1.359(5)$ & $1.344(6)$ \\
\hline C13-C14 & $1.406(3)$ & $1.399(3)$ & $1.392(4)$ & $1.400(5)$ & $1.371(6)$ & $1.358(7)$ \\
\hline C15-C14 & $1.363(3)$ & $1.359(3)$ & $1.352(4)$ & $1.332(5)$ & $1.354(5)$ & $1.357(6)$ \\
\hline C16-C15 & $1.442(3)$ & $1.444(3)$ & $1.449(3)$ & $1.432(4)$ & $1.466(4)$ & $1.468(5)$ \\
\hline C1-C16 & $1.426(2)$ & $1.422(3)$ & $1.417(3)$ & $1.434(4)$ & $1.399(4)$ & $1.393(5)$ \\
\hline
\end{tabular}

* $100 \mathrm{~K}$ data belongs to single-crystal in Table S2. 


\subsection{NMR Spectroscopy}

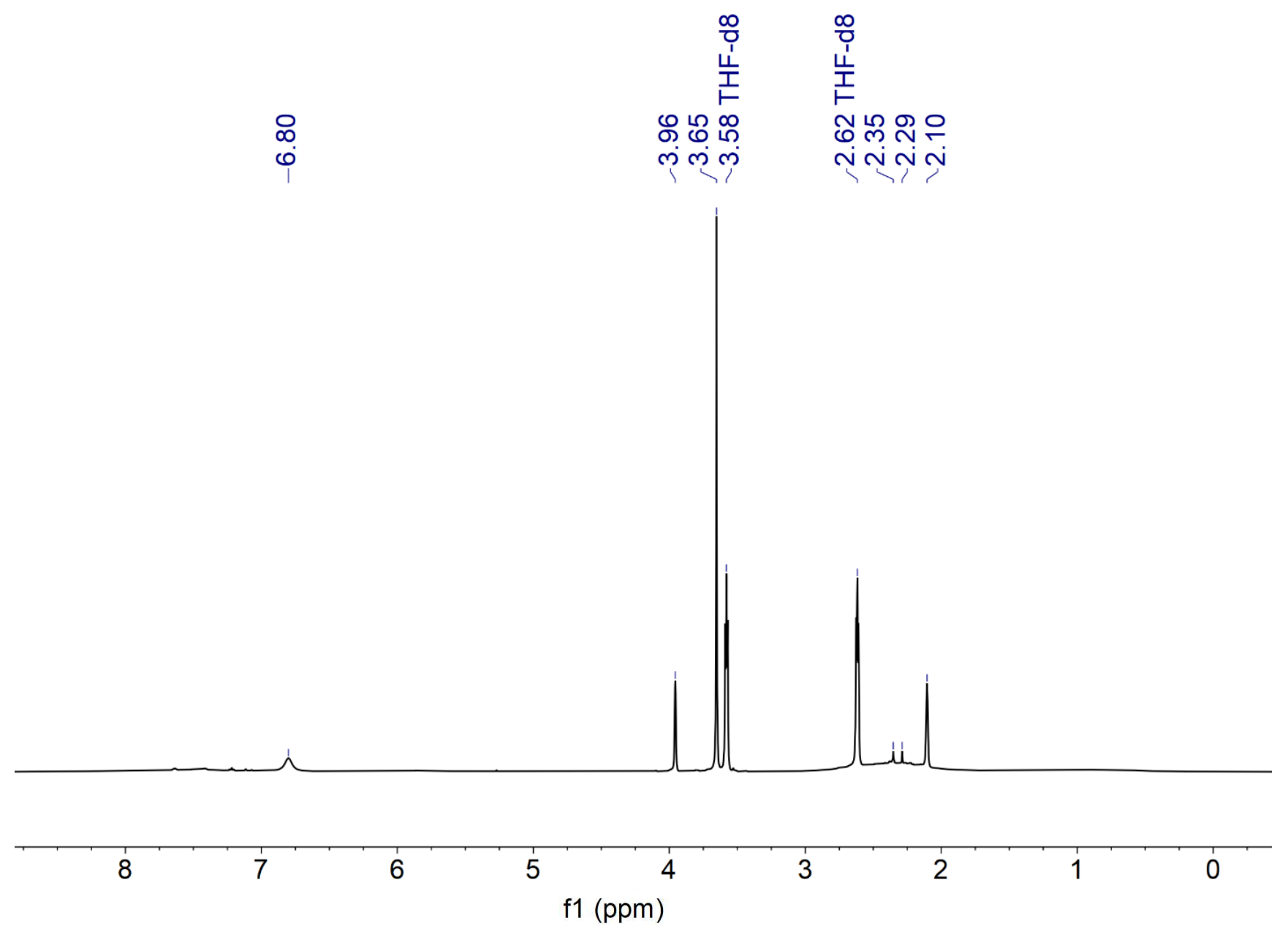

Figure S11. ${ }^{1} \mathrm{H}$ NMR spectrum of $\left[\mathrm{K}(\right.$ crypt-222) $]\left[\mathrm{Cp}^{\mathrm{tet}}{ }_{2} \mathrm{Y}\left(\mathrm{n}^{2}-\mathrm{dbCOT}\right)\right](1),\left(500 \mathrm{MHz}, \mathrm{THF}-\mathrm{d}^{8}\right)$. 
৪ঙ m m

ÑN

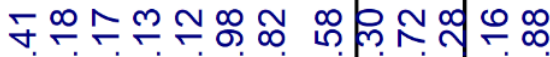

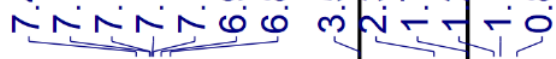

个?

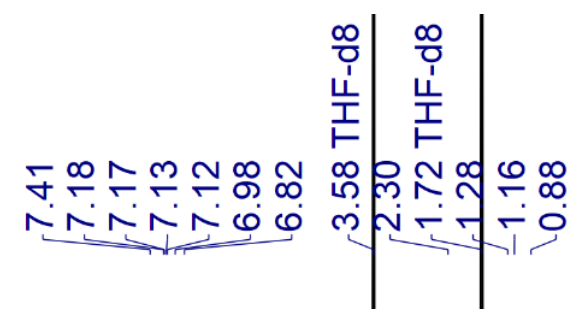

25

20

15

10

5

f1 (ppm)

Figure S12. ${ }^{1} \mathrm{H}$ NMR spectrum of $[\mathrm{K}(\mathrm{crypt}-222)]\left[\mathrm{Cp}^{\text {tet }}{ }_{2} \mathrm{Dy}\left(\eta^{2}-\mathrm{dbCOT}\right)\right](2),\left(500 \mathrm{MHz}, \mathrm{THF}-d^{8}\right)$. 


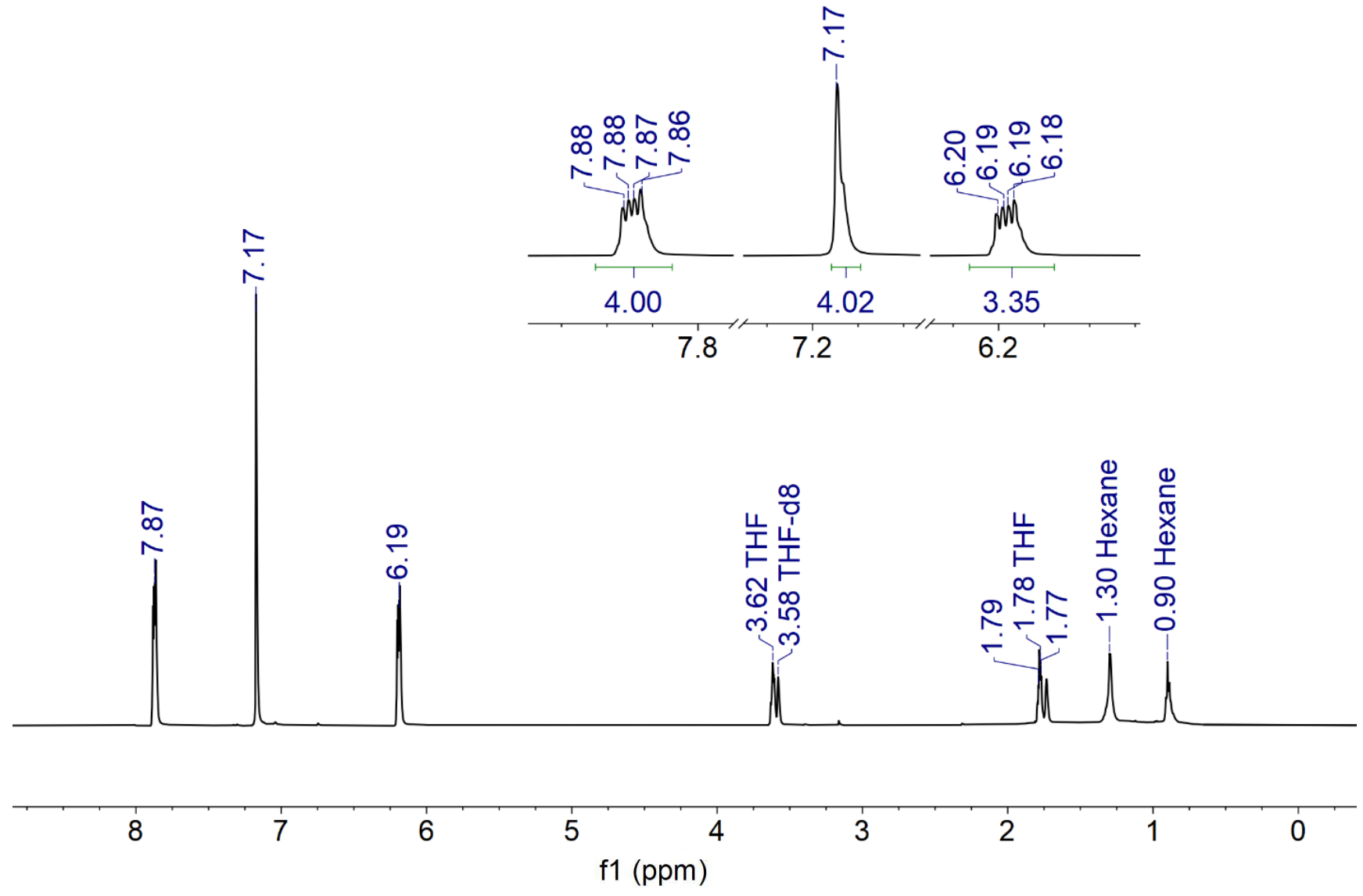

Figure S13. ${ }^{1} \mathrm{H}$ NMR spectrum of $\mathrm{K}_{2} \mathrm{dbCOT}(\mathrm{THF})_{1.5},\left(500 \mathrm{MHz}, \mathrm{THF}-\mathrm{d}^{8}\right)$. 


\subsection{IR Spectroscopy}

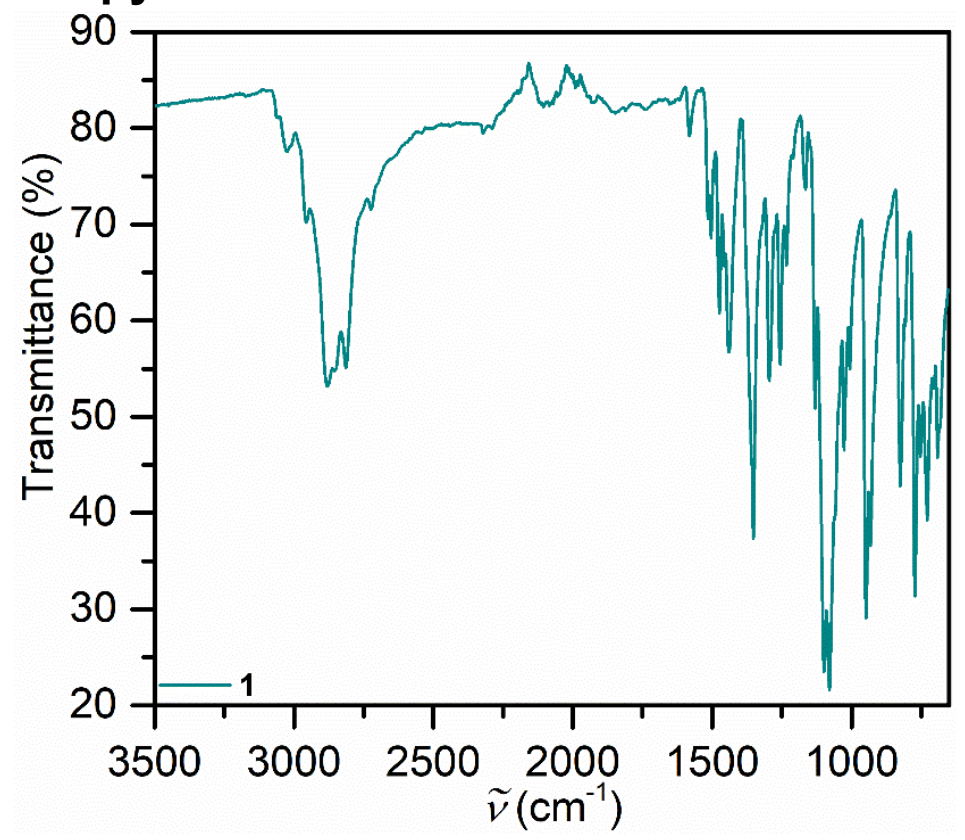

Figure S14. FTIR spectrum of $\left[K(\right.$ crypt-222) $]\left[C p^{\text {tet }}{ }_{2} \mathrm{Y}\left(\eta^{2}-\mathrm{dbCOT}\right)\right]$ (1).

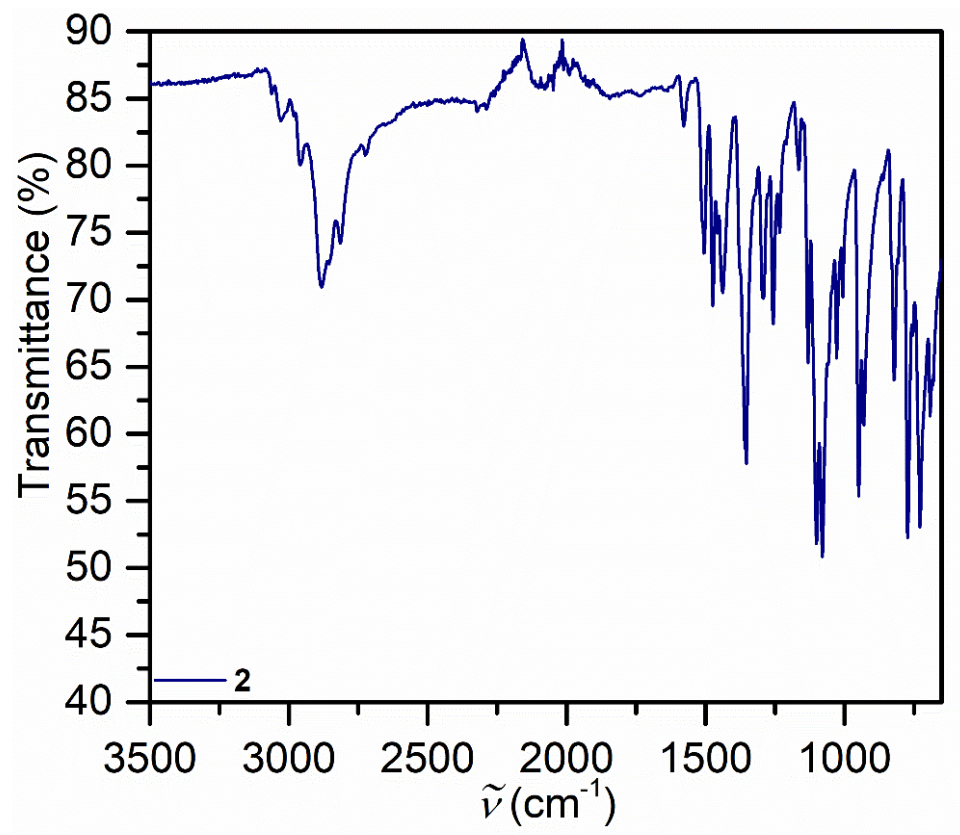

Figure S15. FTIR spectrum of $[K(\operatorname{crypt}-222)]\left[C p^{\text {tet }}{ }_{2} \mathrm{Dy}\left(\eta^{2}-\mathrm{dbCOT}\right)\right](2)$. 


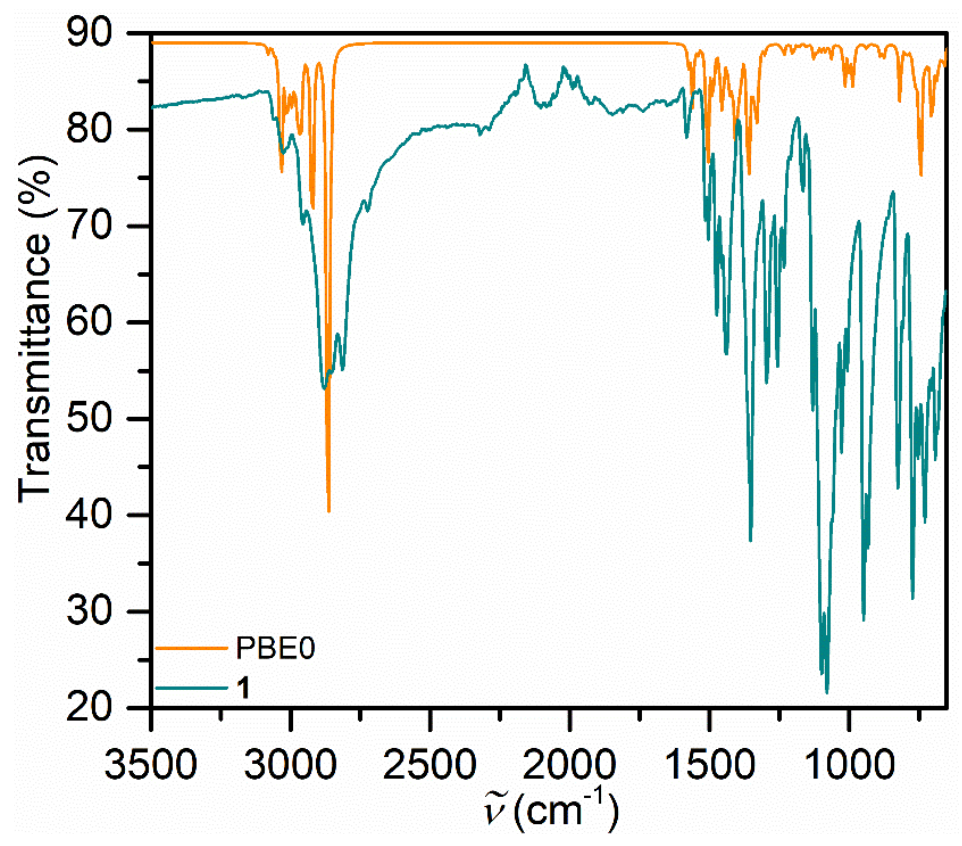

Figure S16. Comparison of the experimental FTIR spectrum of [K(crypt-222)][C $\left.p^{\text {tet }}{ }_{2} \mathrm{Y}\left(\eta^{2}-\mathrm{dbCOT}\right)\right]$ (1) (teal), and calculated stretching frequencies (orange) of $[K(\mathrm{crypt}-222)]\left[\mathrm{Cp}^{\text {tet }}{ }_{2} \mathrm{Y}\left(\mathrm{n}^{2}-\mathrm{dbCOT}\right)\right](1)$, with an applied scaling factor of 0.95 . The calculated spectrum was obtained via DFT with PBE0 functional and def2-TZVP\&6-311G(d,p) basis sets.

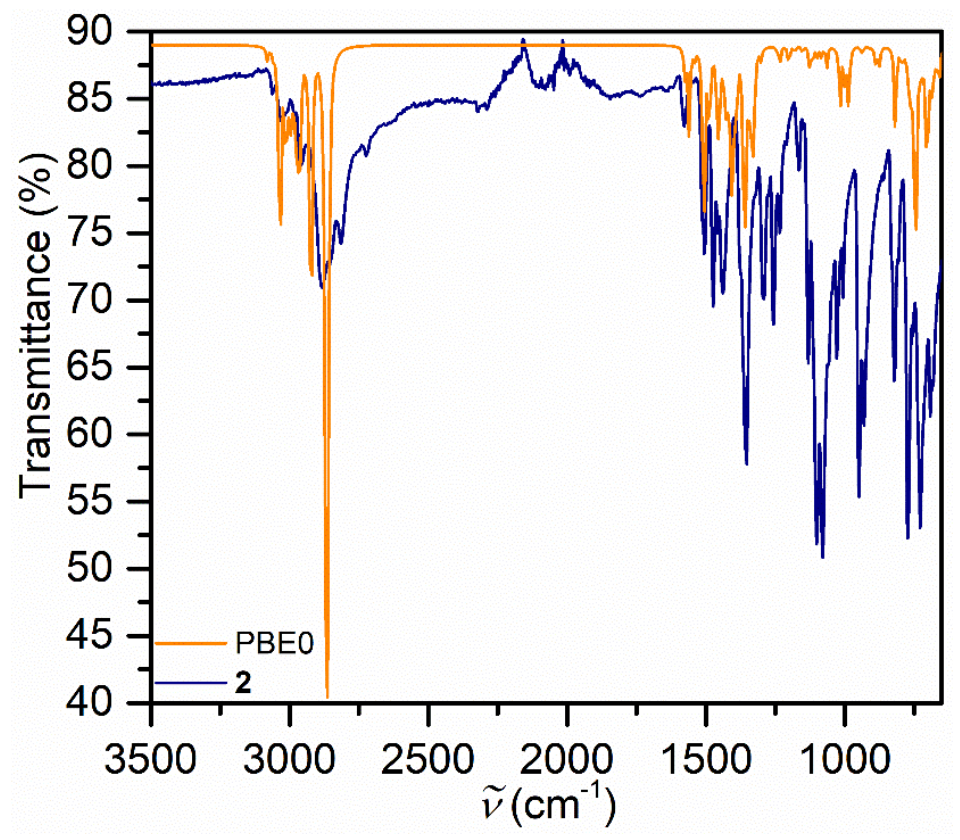

Figure S17. Comparison of the experimental FTIR spectrum of $[K(\mathrm{crypt}-222)]\left[\mathrm{Cp} p^{\text {tet }}{ }_{2} \mathrm{Dy}\left(\eta^{2}\right.\right.$ $\mathrm{dbCOT})]$ (2) (blue), and calculated stretching frequencies (orange) of [K(crypt-222)][Cp ${ }_{2}^{\text {tet }}{ }_{2} \mathrm{Y}\left(\mathrm{n}^{2}-\right.$ $\mathrm{dbCOT})]$ (1), with an applied scaling factor of 0.95 . The calculated spectrum was obtained via DFT with PBE0 functional and def2-TZVP\&6-311G $(d, p)$ basis sets. 


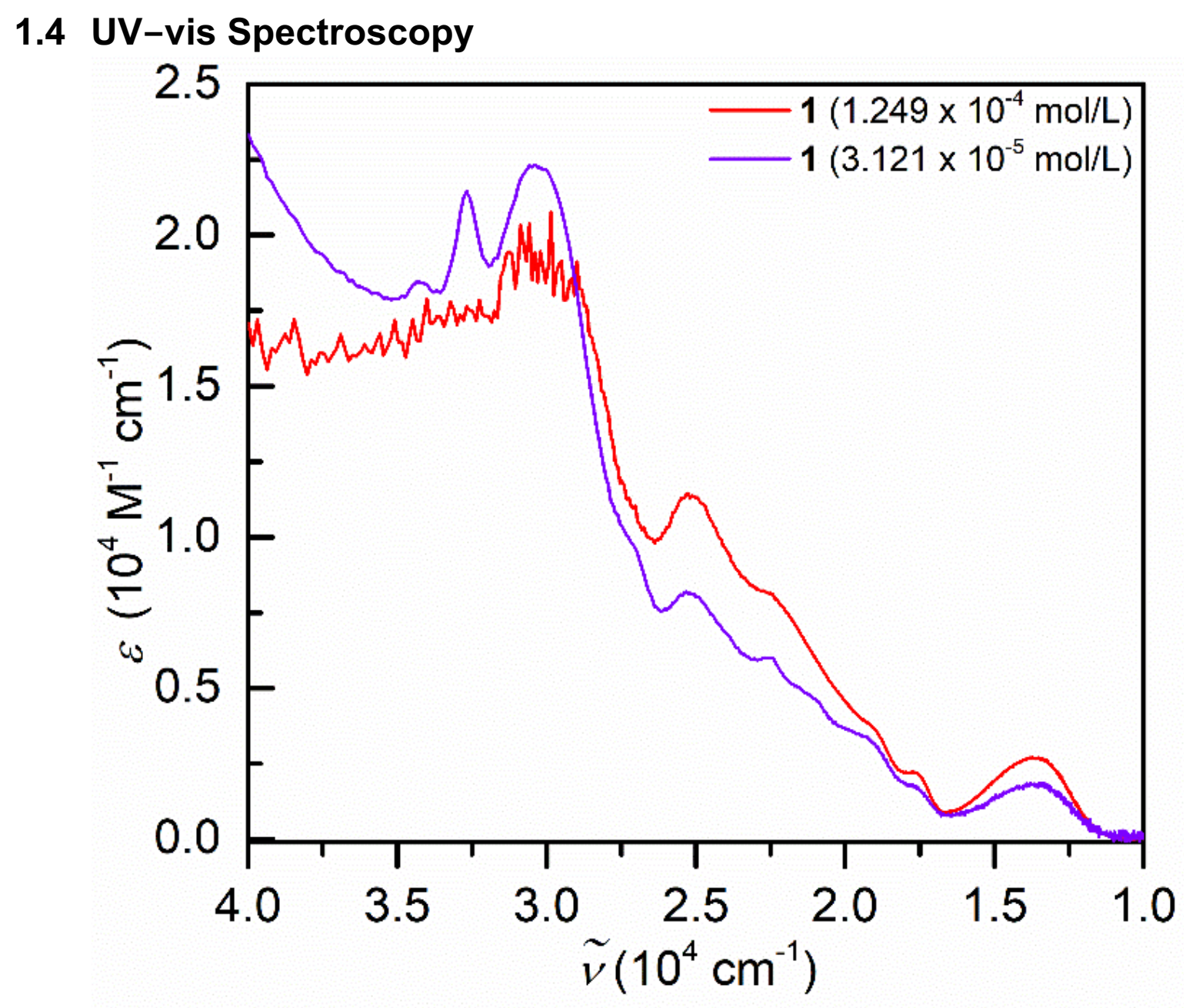

Figure S18. UV-vis spectra of $[\mathrm{K}(\mathrm{crypt}-222)]\left[\mathrm{Cp}^{\text {tet }}{ }_{2} \mathrm{Y}\left(\mathrm{\eta}^{2}-\mathrm{dbCOT}\right)\right](1)$, taken in THF solution at $1.249 \times 10^{-4} \mathrm{~mol} / \mathrm{L}$ and $3.121 \times 10^{-5} \mathrm{~mol} / \mathrm{L}$. 


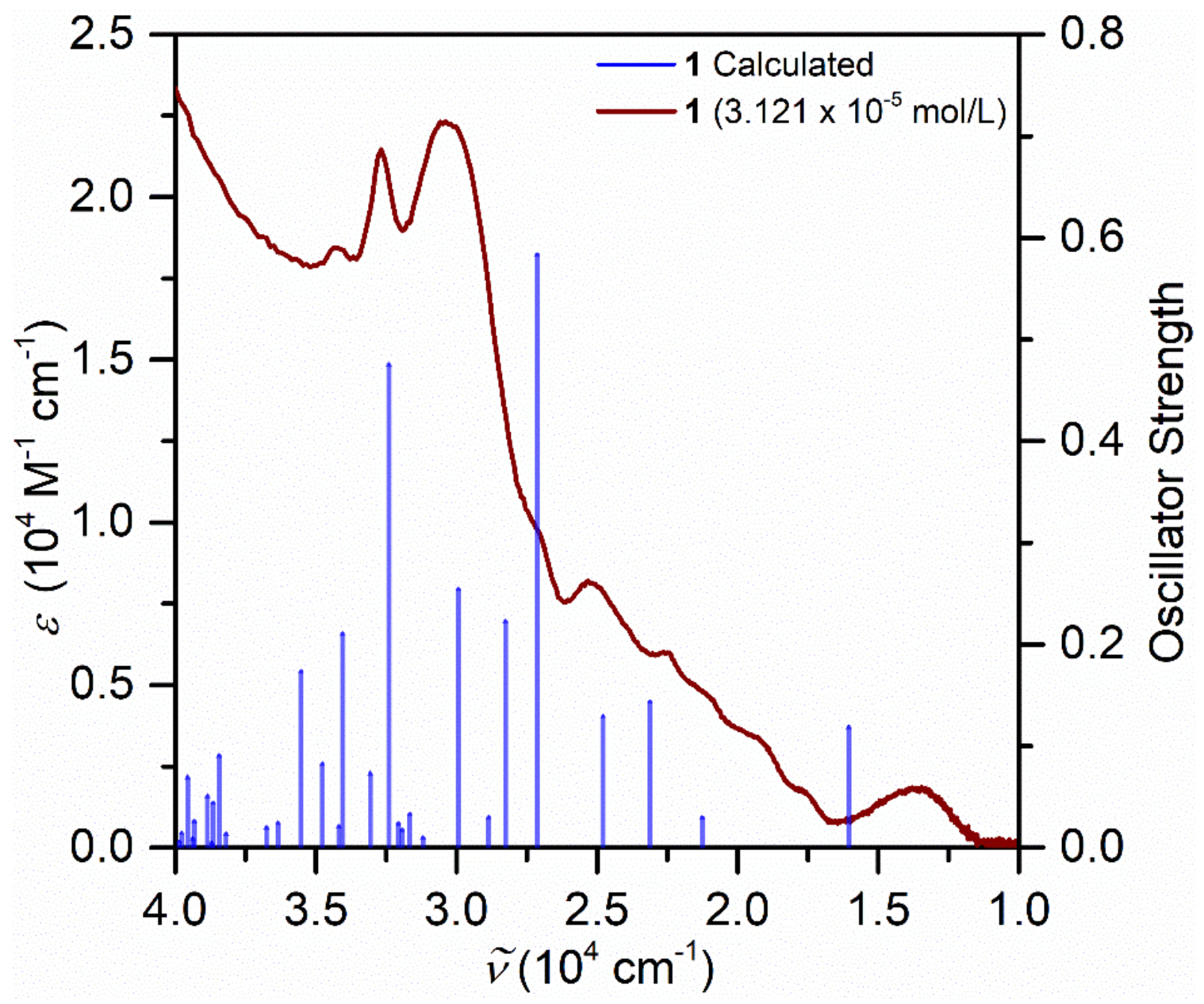

Figure S19. Overlay of the UV-vis spectrum of $[\mathrm{K}(\operatorname{crypt}-222)]\left[C \mathrm{Cp}^{\text {tet }}{ }_{2} \mathrm{Y}\left(\mathrm{n}^{2}-\mathrm{dbCOT}\right)\right](1)$, (dark red line, $3.121 \times 10^{-5} \mathrm{~mol} / \mathrm{L}$ in THF) and transitions calculated from TD-DFT (blue bars). 


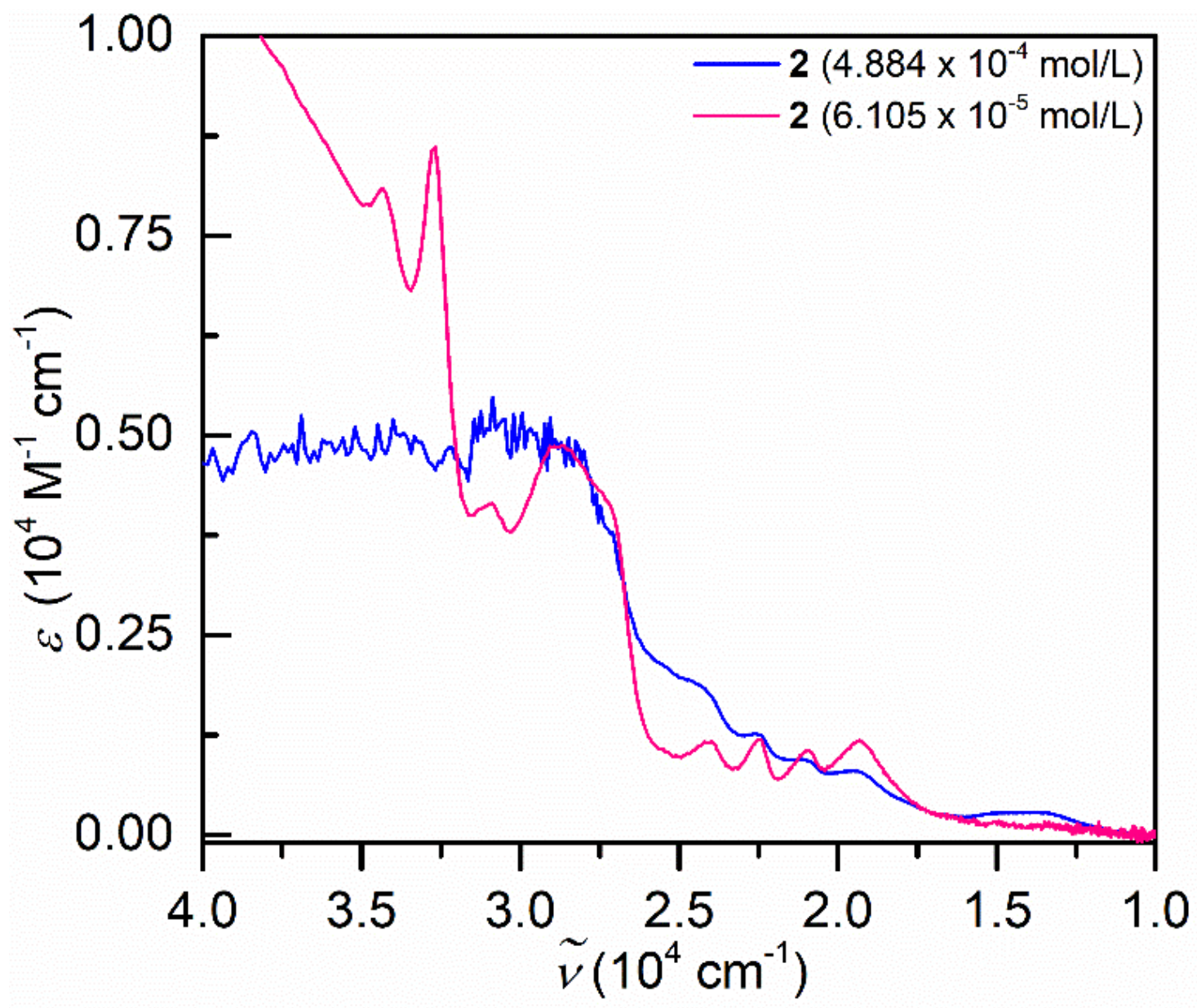

Figure S20. UV-vis spectra of [K(crypt-222)][C $\left.\mathrm{p}^{\text {tet }}{ }_{2} \mathrm{Dy}\left(\mathrm{\eta}^{2}-\mathrm{dbCOT}\right)\right](2)$, taken in THF solution at $4.884 \times 10^{-4} \mathrm{~mol} / \mathrm{L}$ and $6.105 \times 10^{-5} \mathrm{~mol} / \mathrm{L}$. 


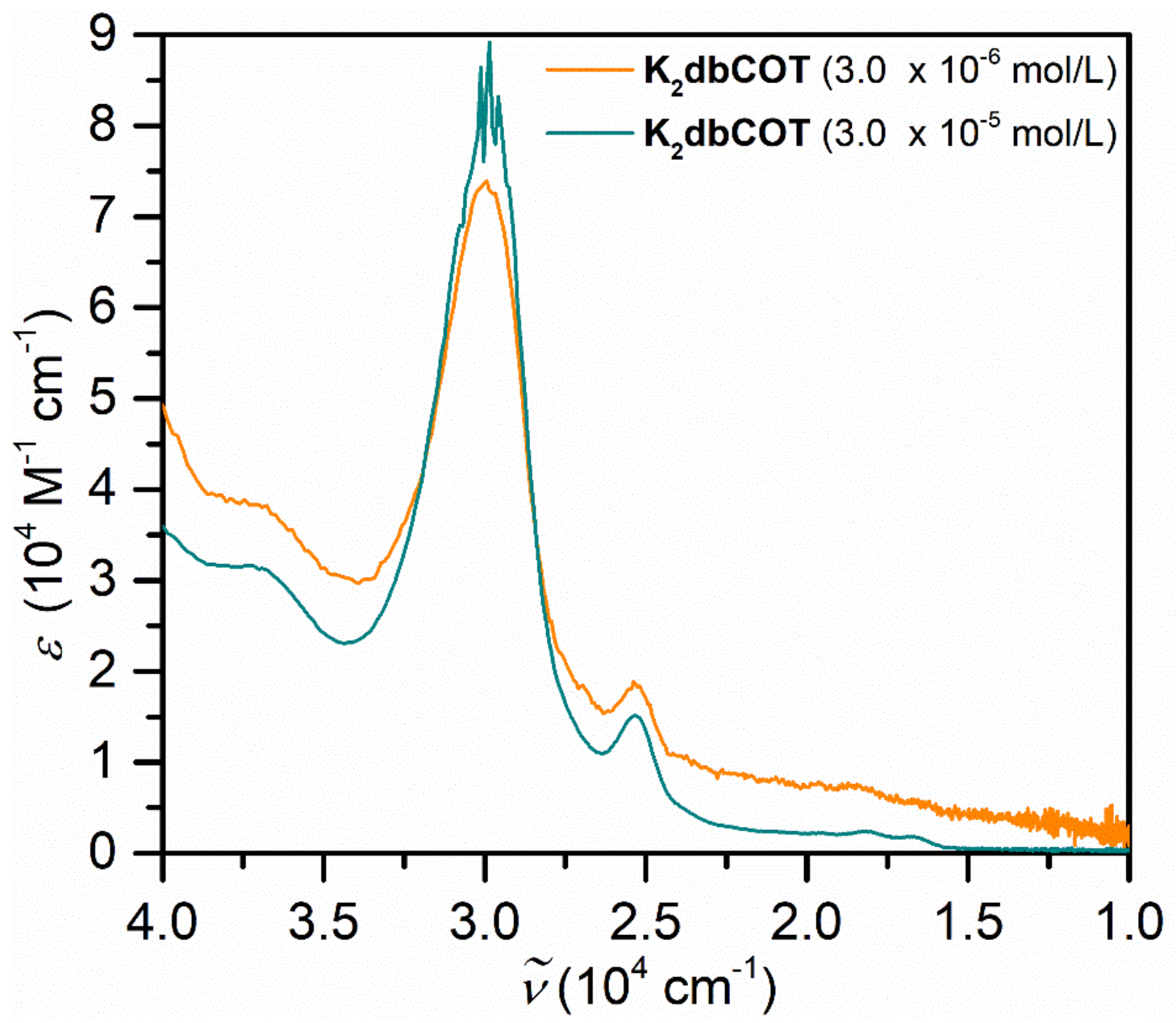

Figure S21. UV-vis spectra of $\mathrm{K}_{2} \mathrm{dbCOT}$, taken in THF solution at $3.0 \times 10^{-6} \mathrm{~mol} / \mathrm{L}$ and $3.0 \times 10^{-5}$ $\mathrm{mol} / \mathrm{L}$. 


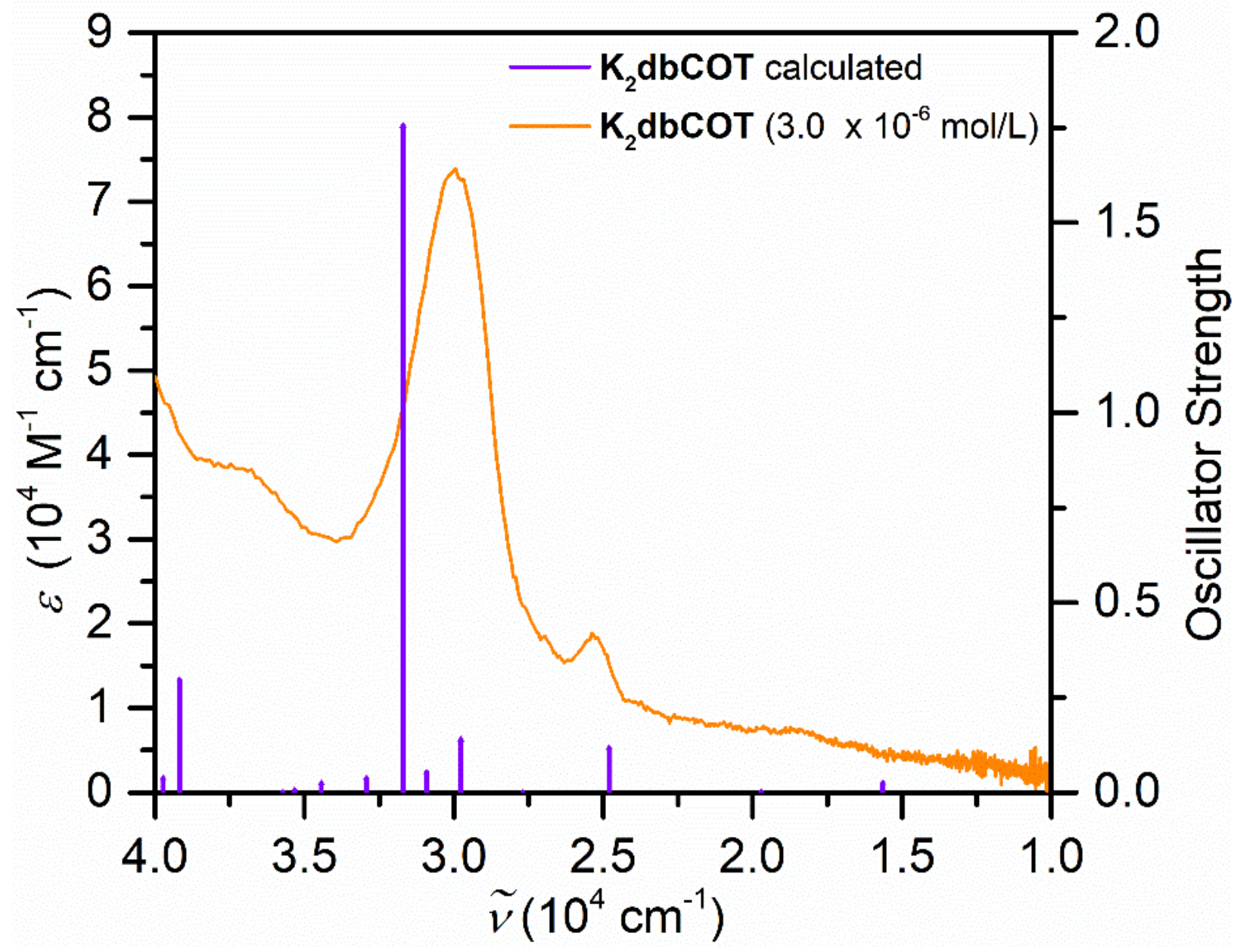

Figure S22. Overlay of UV-vis spectrum of $\mathrm{K}_{2} \mathrm{dbCOT}$, (orange line, $3.0 \times 10^{-6} \mathrm{~mol} / \mathrm{L}$ in THF) and transitions calculated from TD-DFT (purple bars). 


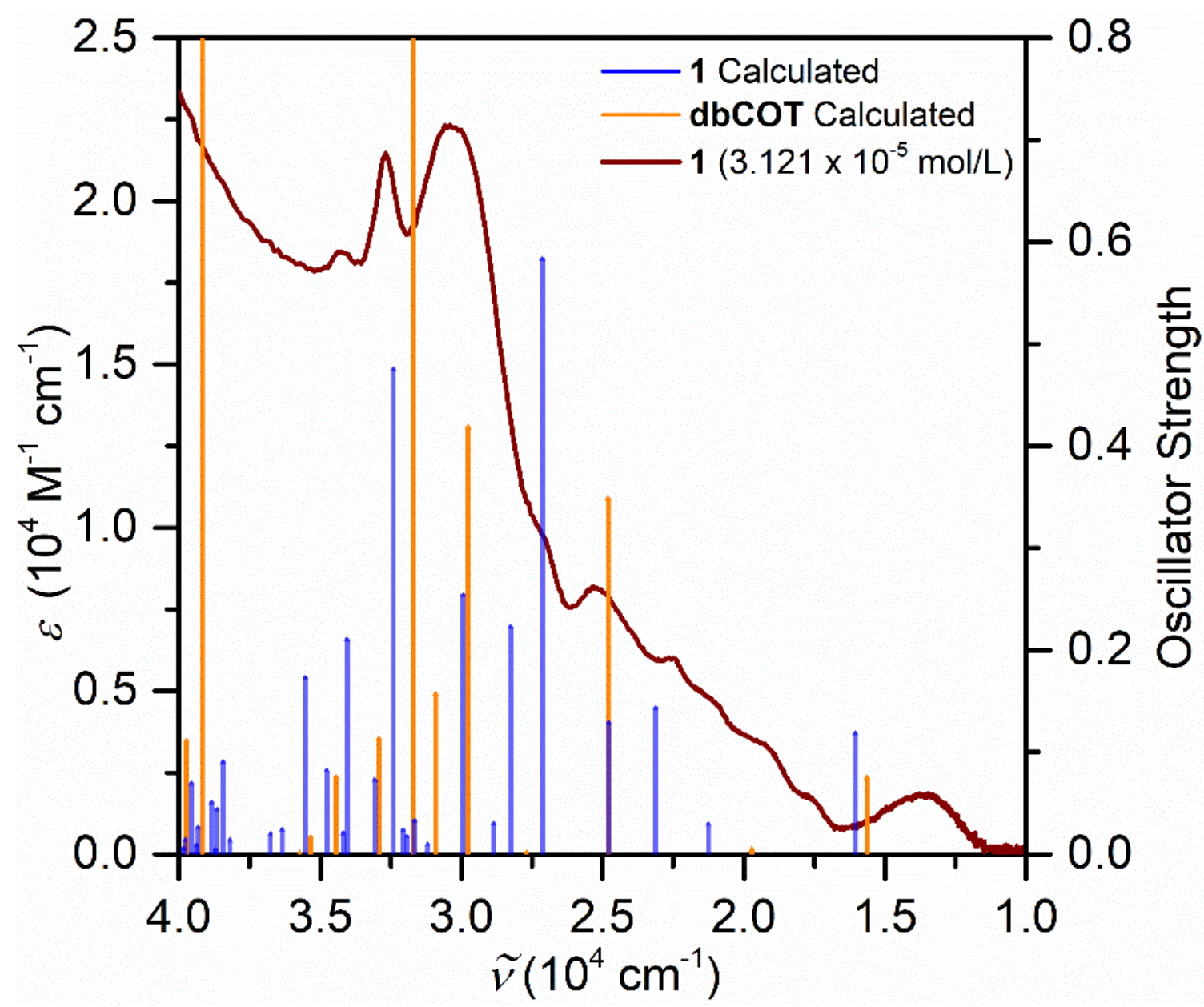

Figure S23. Overlay of UV-vis spectrum of [K(crypt-222)][C $\left.\mathrm{p}_{2}^{\text {tet }} \mathrm{Y}\left(\mathrm{n}^{2}-\mathrm{dbCOT}\right)\right]$ (1), (dark red line, $3.121 \times 10^{-5} \mathrm{~mol} / \mathrm{L}$ in THF) and transitions calculated from TD-DFT for 1 (blue bars) and for $\mathrm{K}_{2} \mathrm{dbCOT}$ (orange bars). The calculated oscillator strengths of $\mathrm{K}_{2} \mathrm{dbCOT}$ were scaled by factor 3 for a better visual distinction. 


\subsection{Electrochemistry}
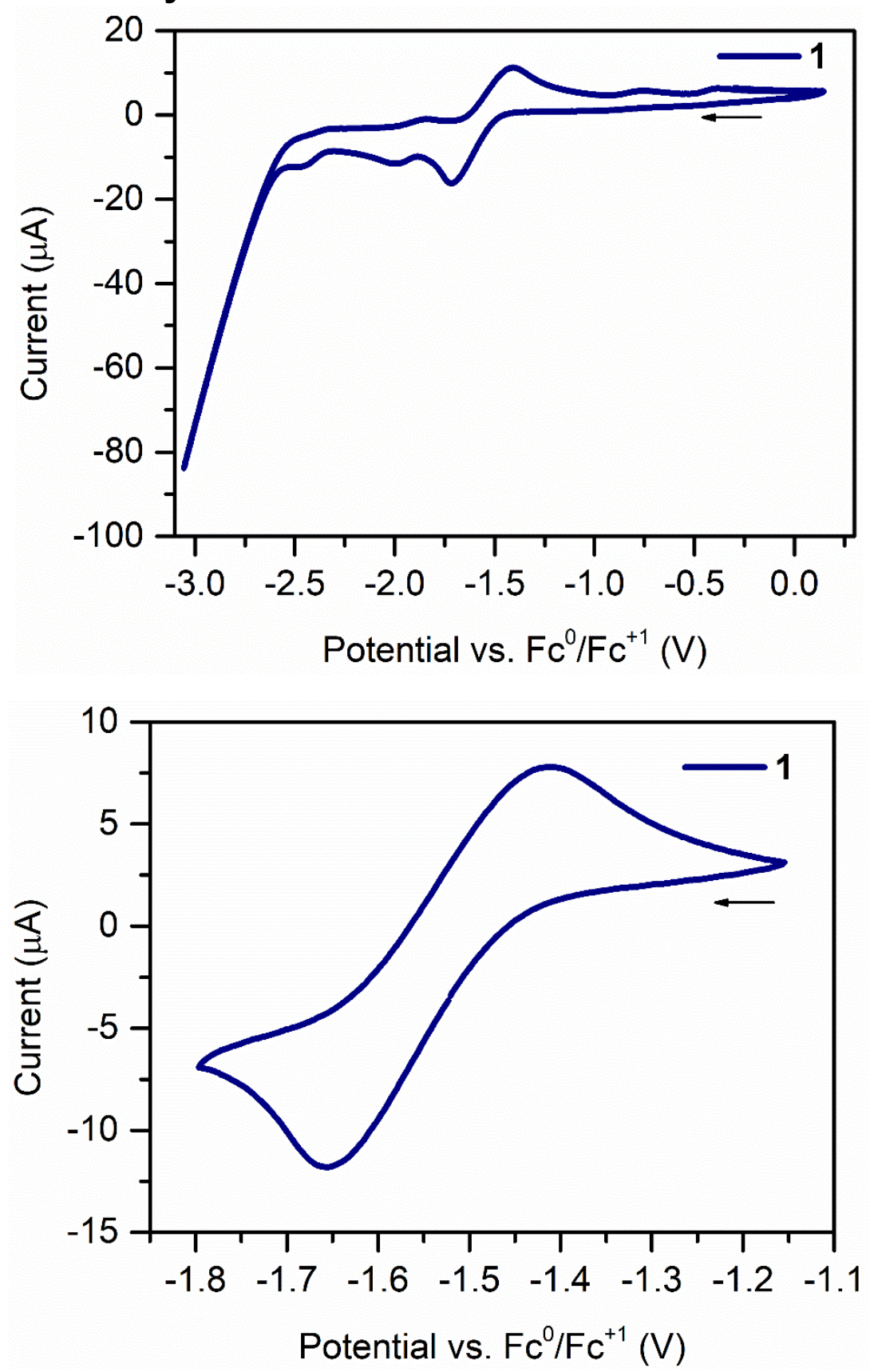

Figure S24. Cyclic voltammograms of $[K(\mathrm{crypt}-222)]\left[C \mathrm{p}^{\text {tet }}{ }_{2} \mathrm{Y}\left(\eta^{2}-\mathrm{dbCOT}\right)\right](1)$, recorded in THF (3 $\mathrm{mM})$ with [ $\left.{ }^{n} \mathrm{Bu}_{4} \mathrm{~N}\right]\left[\mathrm{PF}_{6}\right](250 \mathrm{mM})$ as supporting electrolyte. A scan of the full potential range $(-3.0$ $-0.1 \mathrm{~V}$ ) (top) and magnification of the quasi-reversible feature at $-1.527 \mathrm{~V}$ (bottom) are shown. 

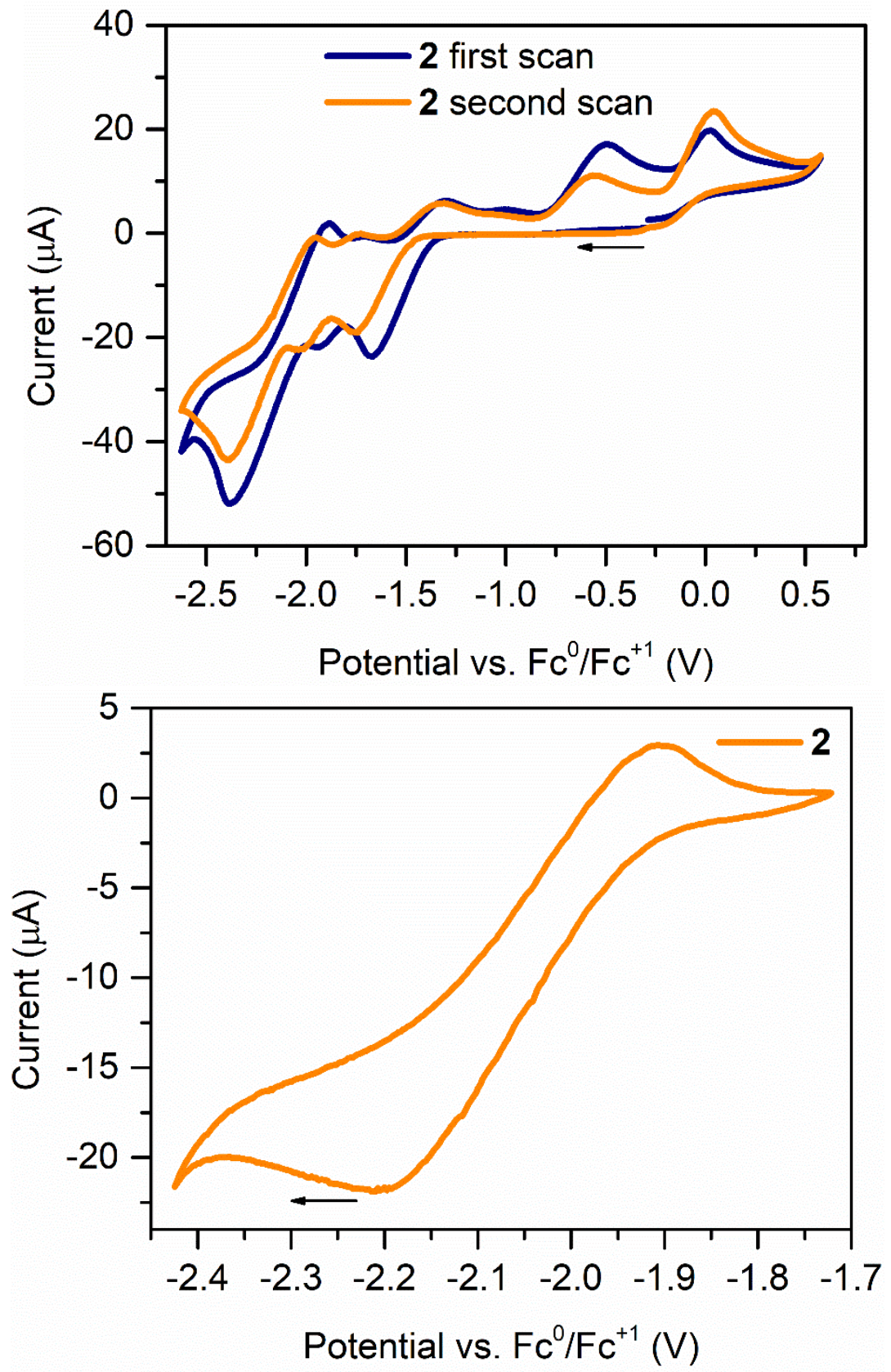

Figure S25. Cyclic voltammograms of $[\mathrm{K}(\mathrm{crypt}-222)]\left[\mathrm{Cp}{ }^{\mathrm{tet}} \mathrm{Dy}\left(\mathrm{n}^{2}-\mathrm{dbCOT}\right)\right](\mathbf{2})$, recorded in THF (3 $\mathrm{mM}$ ) with [ $\left.{ }^{n} \mathrm{Bu}_{4} \mathrm{~N}\right]\left[\mathrm{PF}_{6}\right](250 \mathrm{mM})$ as supporting electrolyte. The first and second full potential range scan $(-2.7-0.6 \mathrm{~V})$ (top) and magnification of the quasi-reversible feature at $-2.054 \mathrm{~V}$ (bottom) are shown. In comparison to the CV measurements of 1, the number of irreversible processes observed for $\mathbf{2}$ is higher, while aside from the feature at $-2.054 \mathrm{~V}$ no other redox events could be resolved. 

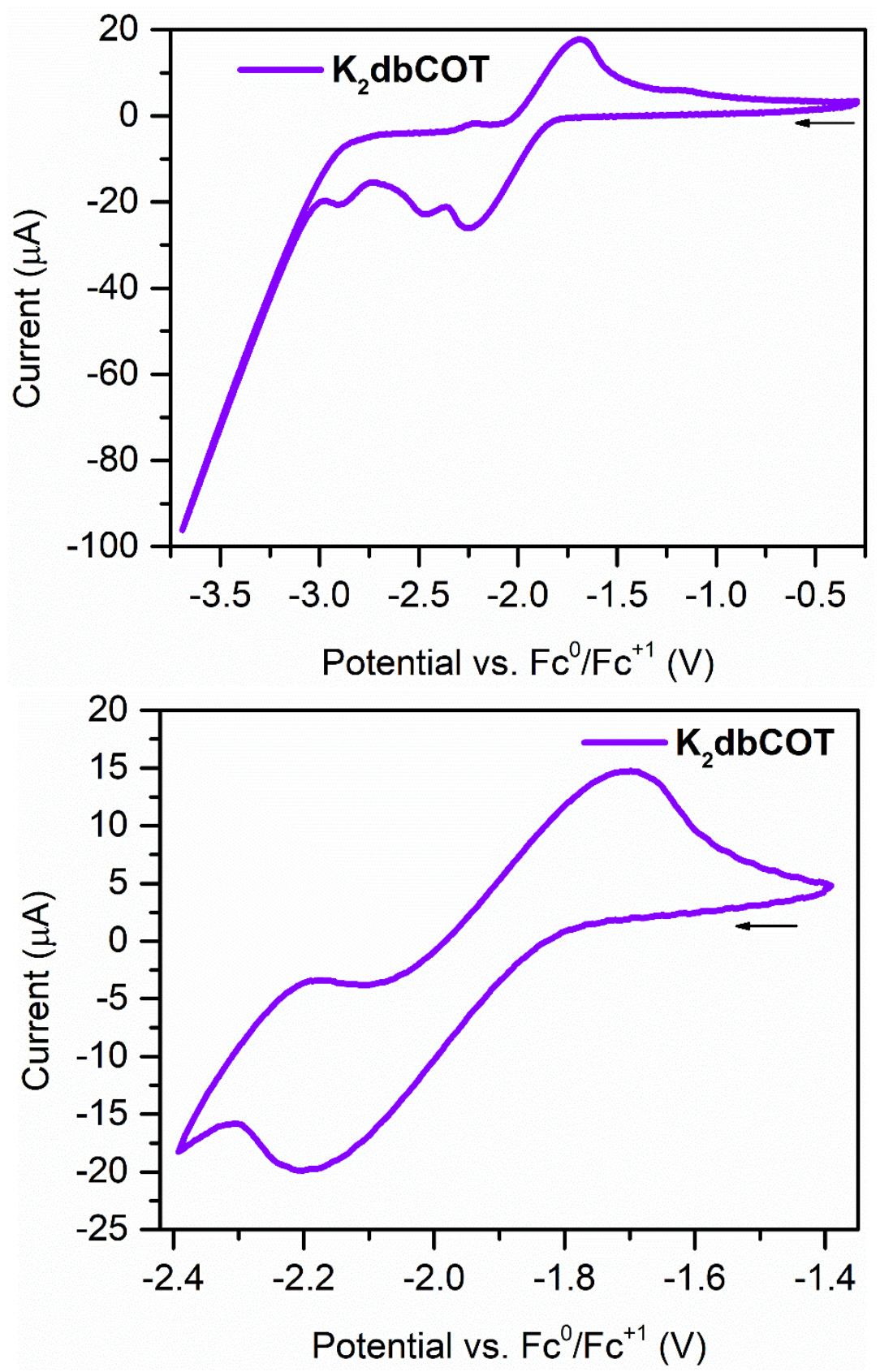

Figure S26. Cyclic voltammograms of $\mathrm{K}_{2} \mathrm{dbCOT}$, recorded in THF $(3 \mathrm{mM})$ with $\left[{ }^{n} \mathrm{Bu}_{4} \mathrm{~N}\right]\left[\mathrm{PF}_{6}\right](250$ $\mathrm{mM}$ ) as supporting electrolyte. A scan of the full potential range $(-3.7--0.29 \mathrm{~V})$ (top) and magnification of the quasi-reversible feature at $-1.991 \mathrm{~V}$ (bottom) are shown. 


\subsection{Magnetic Data}

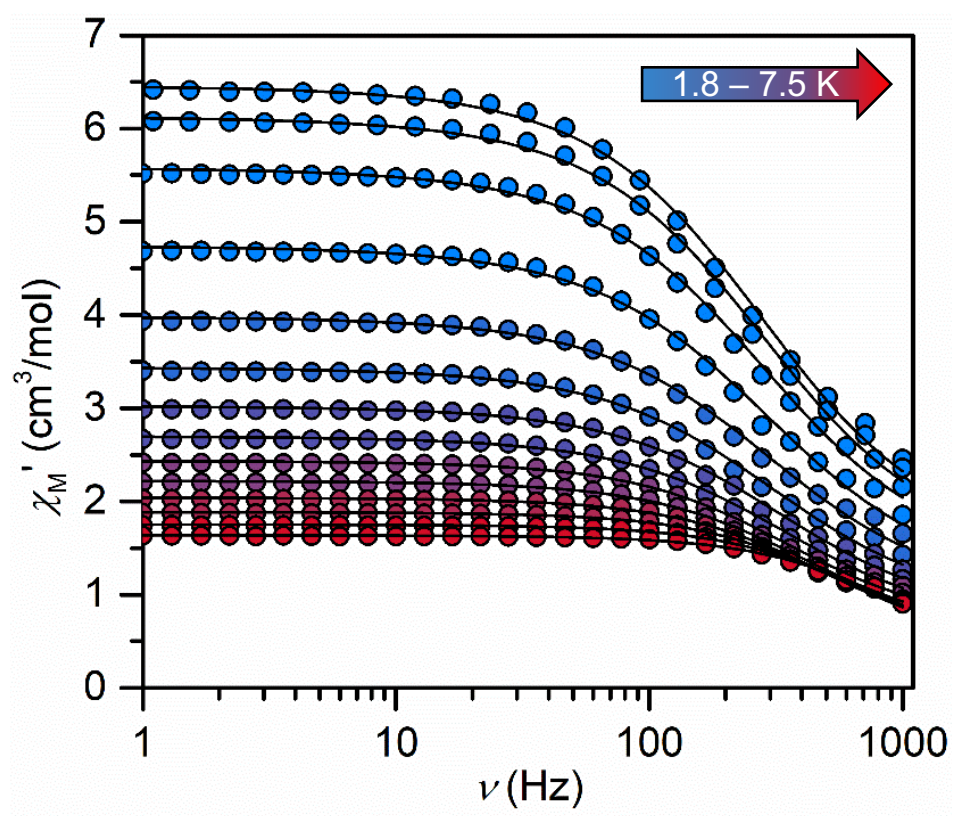

Figure S27. In-phase $\left(\chi \mathrm{m}^{\prime}\right)$ component of the ac magnetic susceptibility for [K(crypt222)][C $\left.\mathrm{p}^{\text {tet }}{ }_{2} \mathrm{Dy}\left(\mathrm{n}^{2}-\mathrm{dbCOT}\right)\right](2)$, under zero applied dc field from $1.8 \mathrm{~K}$ (blue circles) to $7.5 \mathrm{~K}$ (red circles). Solid lines represent fits of the data to a generalized Debye model.

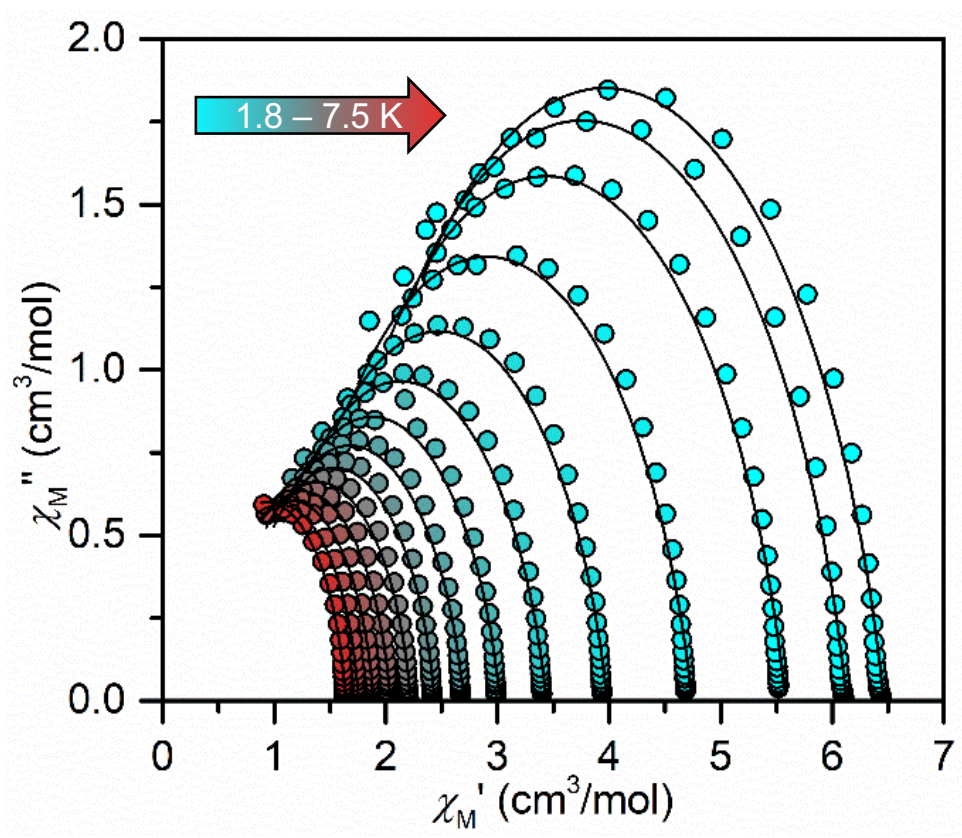

Figure S28. Cole-Cole plots of the ac magnetic susceptibility for $[K(\mathrm{crypt}-222)]\left[\mathrm{C}{ }^{\text {tet }}{ }_{2} \mathrm{Dy}\left(\eta^{2}-\right.\right.$ $\mathrm{dbCOT}$ )] (2), under zero applied dc field from $1.8 \mathrm{~K}$ (teal circles) to $7.5 \mathrm{~K}$ (red circles). Symbols represent the experimental data points and the points representing the fits are connected by solid black lines. 

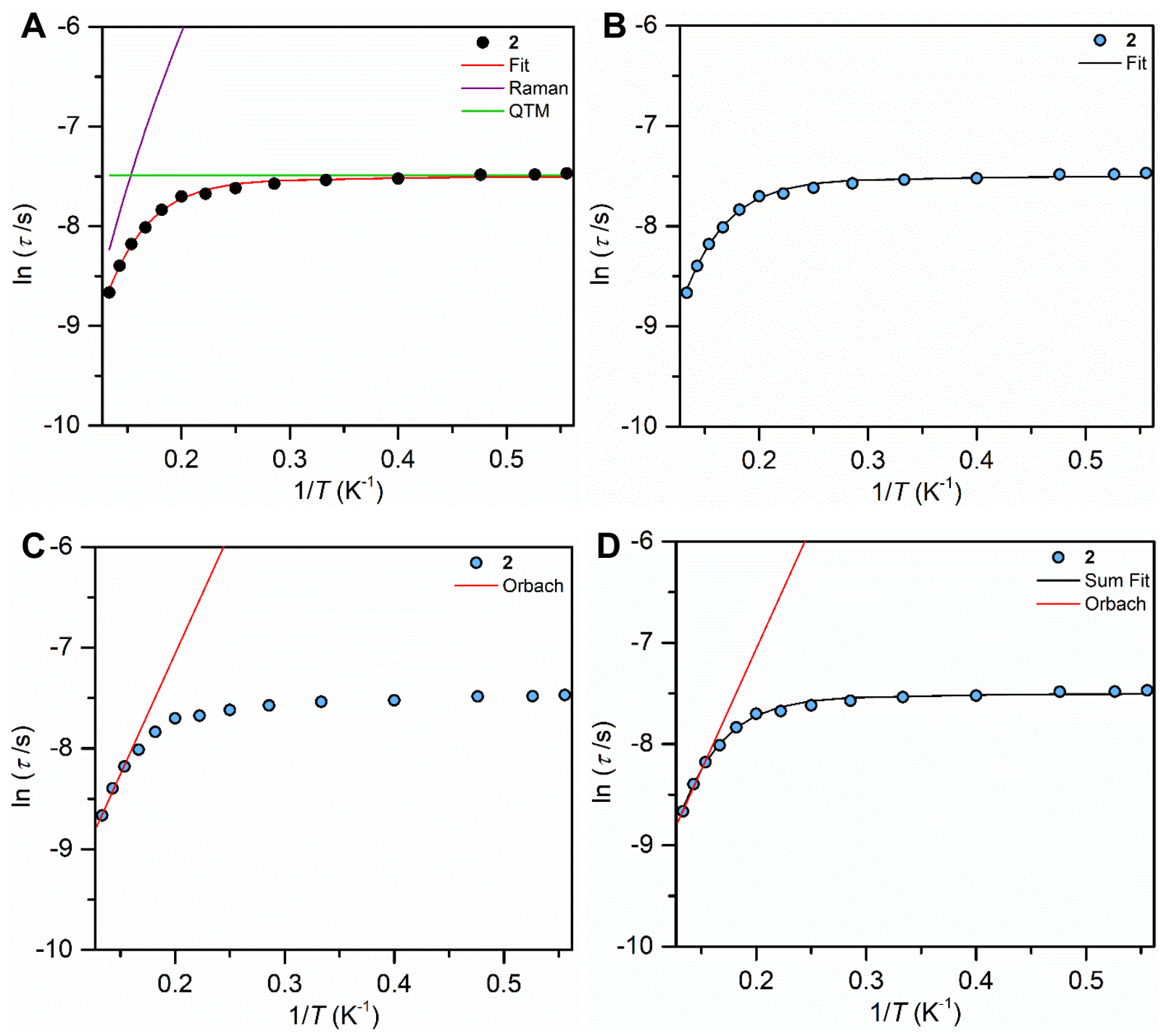

Figure S29. Arrhenius plots of relaxation time data ( $\tau$, colored circles) for complex [K(crypt$222)]\left[C p^{\text {tet }}{ }_{2} \mathrm{Dy}\left(\mathrm{n}^{2}-\mathrm{dbCOT}\right)\right], 2$, at zero applied dc field between 1.8 and $7.5 \mathrm{~K}$. The lines correspond to fits considering various relaxation processes. (A) The purple line and green line represent fits to a Raman and QTM process, respectively, and are contributions to the best fit (red line) to the relaxation times over the entire probed temperature range. (B) The sum fit (black line) arising from fits to Raman and QTM yielded $C=10^{-1} \mathrm{~s}^{-1} \mathrm{~K}^{-n}, n=5$, and $\tau_{\text {QTM }}=10^{-3.3} \mathrm{~s}$. (C) The red line corresponds to fits of the relaxation times observed at the three highest temperatures (6.5 - 7.5 $\mathrm{K})$ to an Orbach process affording $U_{\text {eff }}=20 \mathrm{~K}$ and $\tau_{0}=10^{-5} \mathrm{~s}$. (D) Comparison of the fits to all relaxation times, $\tau$, taking into account Raman and QTM processes, and to relaxation times, $\tau$, observed at high temperatures which were fit to an Orbach process only. 


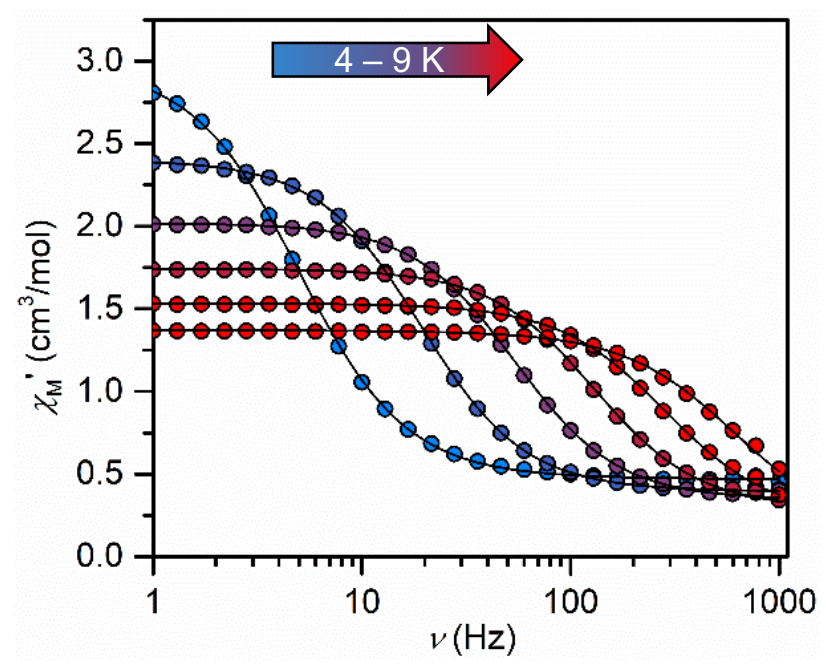

Figure S30. In-phase $\left(\chi_{\mathrm{M}}{ }^{\prime}\right)$ component of the ac magnetic susceptibility for [K(crypt222)][C $\left.\mathrm{Cp}_{2 \mathrm{tet}} \mathrm{Dy}\left(\eta^{2}-\mathrm{dbCOT}\right)\right](2)$, under 1000 Oe applied dc field from $4 \mathrm{~K}$ (blue circles) to $9 \mathrm{~K}$ (red circles). Solid lines represent fits of the data to a generalized Debye model.

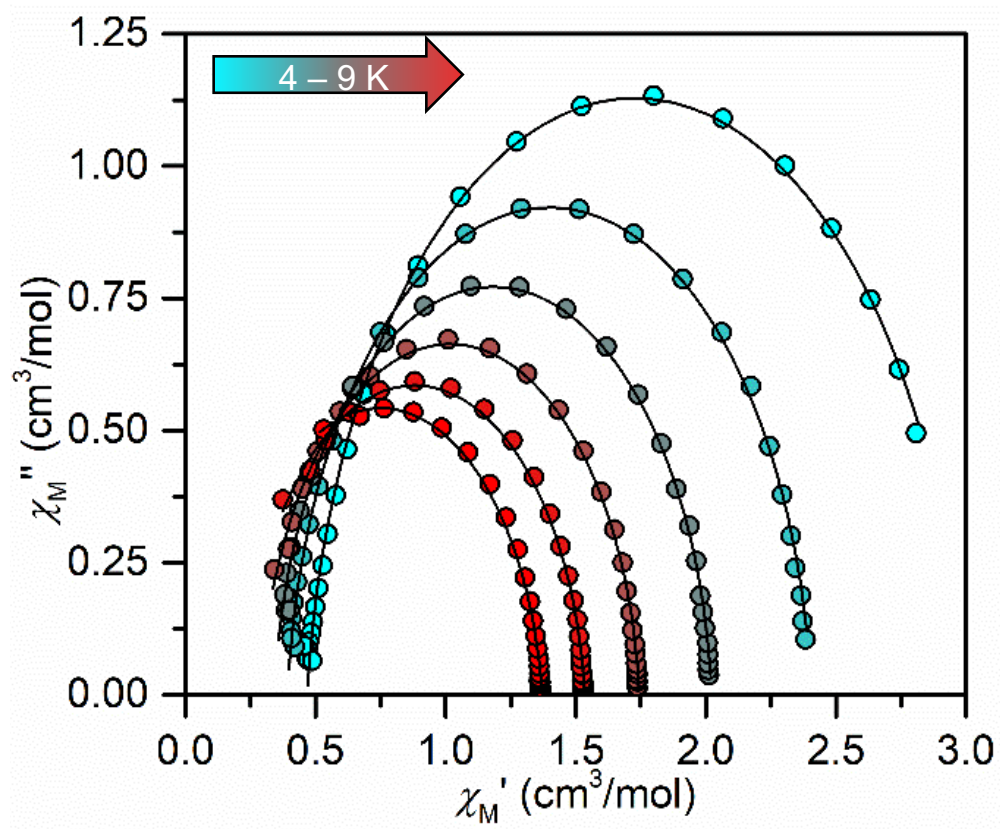

Figure S31. Cole-Cole plots of the ac magnetic susceptibility for $[K(\operatorname{crypt}-222)]\left[C p^{\text {tet }}{ }_{2} D y\left(\eta^{2}-\right.\right.$ $\mathrm{dbCOT}$ )] (2), collected from $4 \mathrm{~K}$ (teal circles) and $9 \mathrm{~K}$ (red circles), under 1000 Oe applied dc field. Symbols represent the experimental data points and the points representing the fits are connected by solid black lines. 

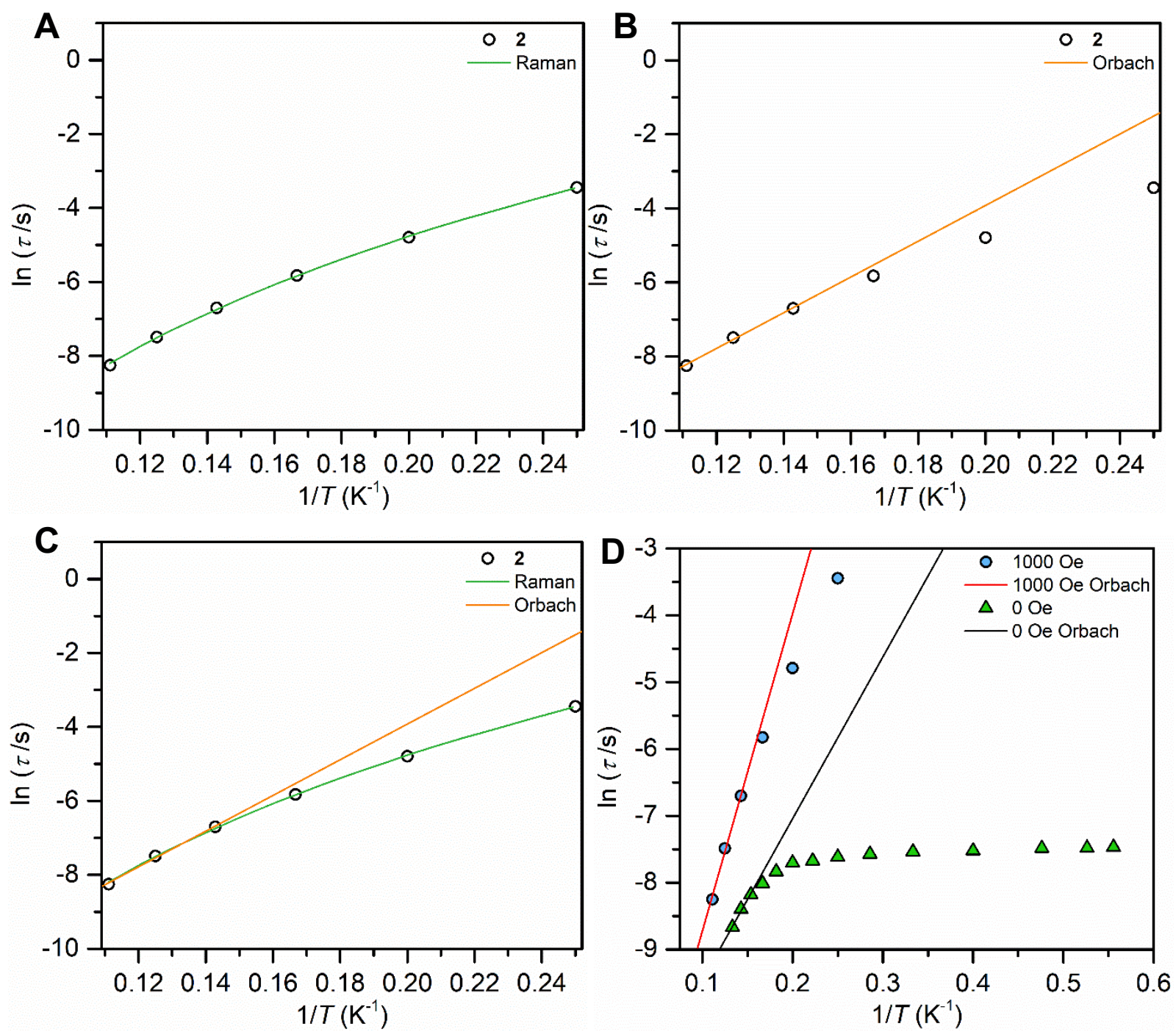

Fig S32. Arrhenius plots of relaxation time, $\tau$, data for [K(crypt-222)][C $\left.p^{\text {tet }}{ }_{2} D y\left(\eta^{2}-d b C O T\right)\right](2)$, under 1000 Oe applied dc field between 4 and $9 \mathrm{~K}$. The lines correspond to fits considering various relaxation processes. (A) The green line represents the best fit to the relaxation times observed between 4 and $9 \mathrm{~K}$ to a Raman process giving rise to $C=10^{-2.0} \mathrm{~s}^{-1} \mathrm{~K}^{-n}$ and $n=5.9$. (B) The orange line corresponds to a fit of the $\tau$ monitored at the three highest temperatures $(7-9 \mathrm{~K})$ to an Orbach process yielding $U_{\text {eff }}=50 \mathrm{~K}$ and $\tau_{0}=10^{-6} \mathrm{~s}$. (C) Comparison of the obtained fits to Orbach (orange) and Raman (green) relaxation processes, respectively. (D) Comparison of the fits to the relaxation times, $\tau$, observed at high temperatures to an Orbach process, under $0 \mathrm{Oe}$ (green triangles) and $1000 \mathrm{Oe}$ (blue circles) applied dc field. 

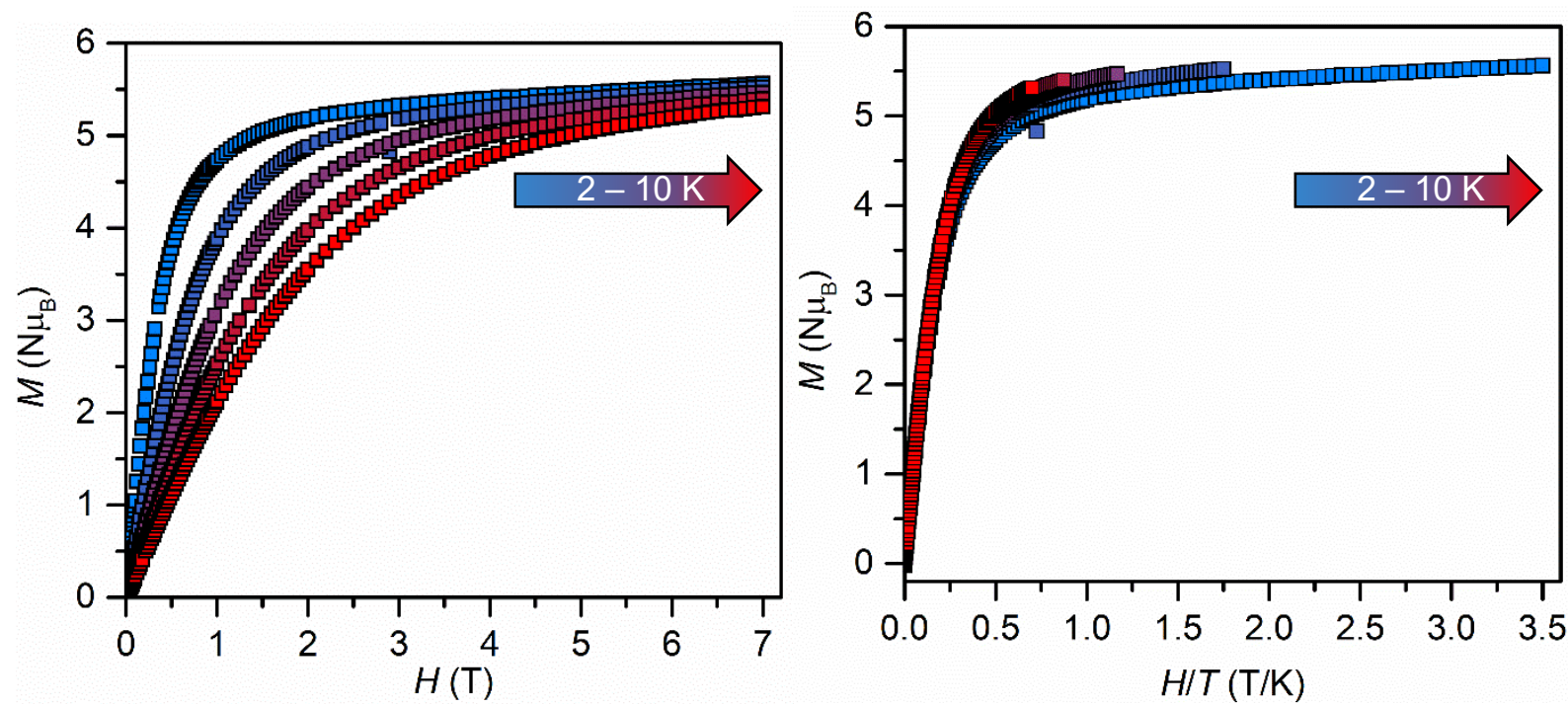

Figure S33. Field-dependent magnetization (left) and reduced magnetization (right) data for $[\mathrm{K}(\mathrm{crypt}-222)]\left[\mathrm{Cp}^{\text {tet }}{ }_{2} \mathrm{Dy}\left(\eta^{2}-\mathrm{dbCOT}\right)\right](2)$, recorded between 0 and $7 \mathrm{~T}$ from $2 \mathrm{~K}$ (blue squares) and $10 \mathrm{~K}$ (red squares). 

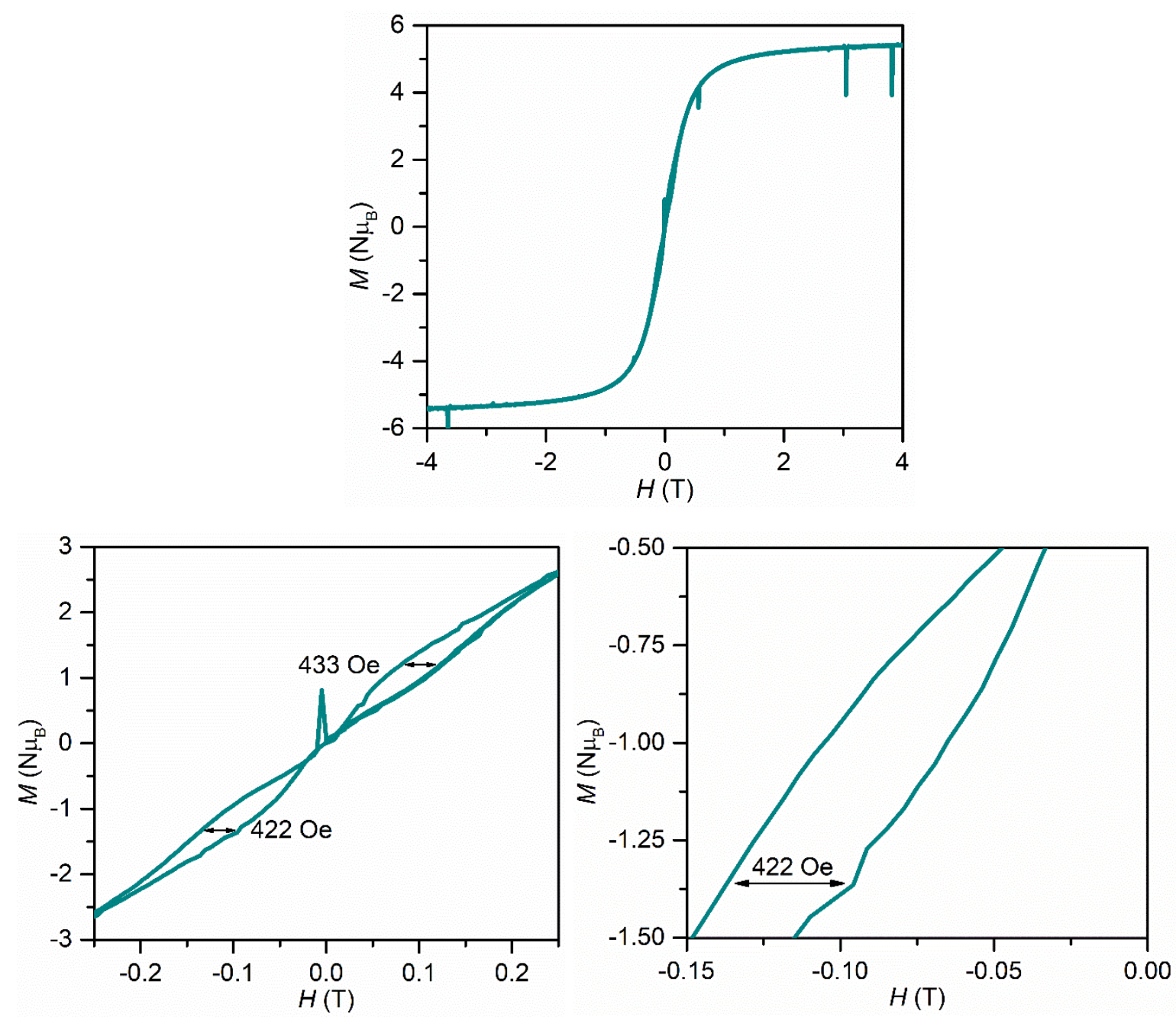

Figure S34. (Top) Variable-field magnetization data for [K(crypt-222)][Cp $\left.{ }_{2}^{\text {tet }} \mathrm{Dy}\left(\eta^{2}-\mathrm{dbCOT}\right)\right](2)$, collected at $1.8 \mathrm{~K}$ between -4 and $+4 \mathrm{~T}$ at an average sweep rate of $100 \mathrm{Oe} / \mathrm{s}$. Hysteresis data is shown from $-0.25 \mathrm{~T}$ and $+0.25 \mathrm{~T}$ (bottom left), and $-0.15 \mathrm{~T}$ and $0 \mathrm{~T}$ (bottom right). 


\subsection{Calculated Orientation of the Magnetic Moment}

The orientation of the magnetic moment of the $\left[\mathrm{Cp}^{\text {tet }}{ }_{2} \mathrm{Dy}\left(\eta^{2}-\mathrm{dbCOT}\right)\right]^{-}$anion in 2 was calculated based of the electrostatic forces imposed by the ligand scaffold with the program MAGELLAN. ${ }^{2}$ Different charge distributions on the $\mathrm{dbCOT}^{2-}$ ligand were investigated to elucidate the most realistic representation. The following empirical charge distributions were considered: $(A)-1$ charges localized on the coordinating carbon atoms and no further delocalization, $(B)$ charge delocalization on the COT $\left(\mathrm{C}_{8}\right)$ moiety only, and $(\mathrm{C})$ evenly distributed over all $\mathrm{dbCOT}$ carbon atoms. Furthermore, we considered the charge delocalization as obtained from DFT calculations on the $\left[\mathrm{Cp}^{\text {tet }}{ }_{2} \mathrm{Y}\left(\eta^{2}-\mathrm{dbCOT}\right)\right]^{-}$anion of 1 , for $(\mathrm{D}) \mathrm{NBO}$ charges and $(\mathrm{E})$ Mulliken charges ( $\mathrm{H}$ atom charges summed into the carbon atoms), where the charges were found to gradually decrease with increasing distance to the coordinating carbon atoms. An overview over the respective charges per model is given in Table S7. Graphical representations of the calculated preferred orientations are given in Figure S35. The outcome indicates that the consideration of charge delocalization on the dbCOT ligand has a significant impact on the magnetic axis: While the sole localization of negative charge on the coordinating carbon atoms results in the magnetic moment being dominated by the dbCOT ligand on oriented towards it, the varying degrees of charge delocalization examined generates a diminishing impact of the dbCOT charges. The orientation of the magnetic moments in all charge delocalized cases are dominated by the $\left(\mathrm{Cp}_{2}\right)$ ligand framework.

Table S7. Overview of different charge localization schemes employed for the calculation of the orientation of the magnetic moment in the $\left[\mathrm{Cp}^{\text {tet }}{ }_{2} \mathrm{Dy}\left(\eta^{2}-\mathrm{dbCOT}\right)\right]^{-}$anion in (2).

\begin{tabular}{c|ccccc}
\hline Carbon & Model (A) & Model (B) & Model (C) & $\begin{array}{c}\text { Normalized } \\
\text { NBO }\end{array}$ & $\begin{array}{c}\text { Normalized } \\
\text { Mulliken }\end{array}$ \\
\hline${ }^{*}$ C1 & -1 & -0.25 & -0.125 & -0.247 & -0.088 \\
${ }^{*}$ C2 & -1 & -0.25 & -0.125 & -0.251 & -0.387 \\
C3 & 0 & -0.25 & -0.125 & -0.034 & 0.016 \\
C4 & 0 & 0 & -0.125 & -0.108 & -0.096 \\
C5 & 0 & 0 & -0.125 & -0.133 & -0.115 \\
C6 & 0 & 0 & -0.125 & -0.151 & -0.131 \\
C7 & 0 & 0 & -0.125 & -0.095 & -0.080 \\
C8 & 0 & -0.25 & -0.125 & -0.067 & -0.134 \\
C9 & 0 & -0.25 & -0.125 & -0.126 & -0.073 \\
C10 & 0 & -0.25 & -0.125 & -0.133 & -0.112 \\
C11 & 0 & -0.25 & -0.125 & -0.059 & -0.064 \\
C12 & 0 & 0 & -0.125 & -0.091 & -0.077 \\
C13 & 0 & 0 & -0.125 & -0.151 & -0.143 \\
C14 & 0 & 0 & -0.125 & -0.119 & -0.099 \\
C15 & 0 & 0 & -0.125 & -0.144 & -0.151 \\
C16 & 0 & -0.25 & -0.125 & -0.084 & -0.265 \\
\hline
\end{tabular}

${ }^{*} \mathrm{C}$ atoms represent the coordinating carbon atoms. Model $(\mathbf{A})$ represents the sole charge localization on the coordinating carbon atoms, model $(B)$ represents the delocalized charge on the COT ring $\left(\mathrm{C}_{8}\right)$, and model (C) represents an evenly distributed charge over the entire $(\mathrm{dbCOT})^{2^{-}}$ligand. The atom labels coincide with the crystallographic numbers given in Figure S2 and Table S5. 


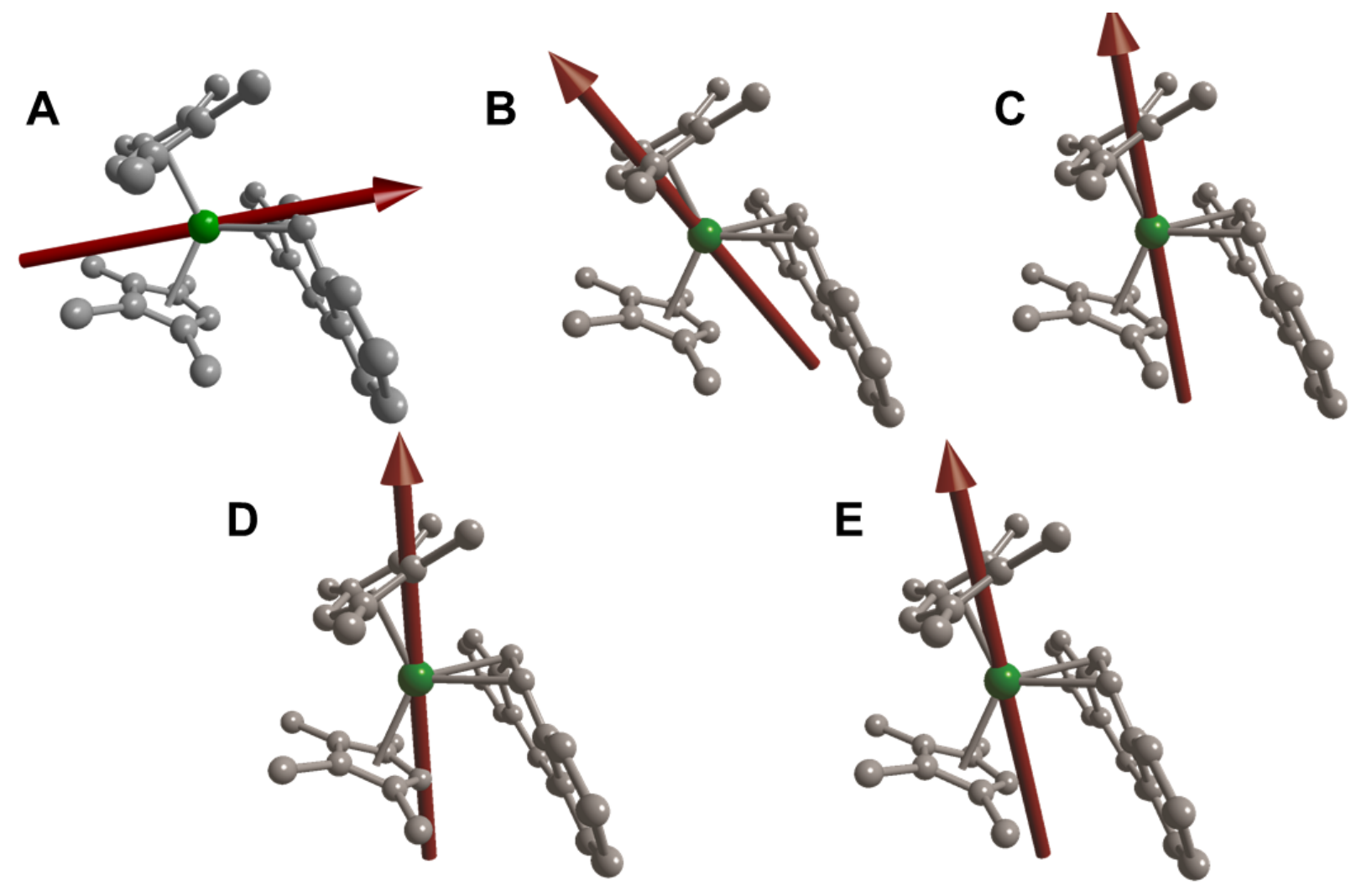

Figure S35. Graphical representation of the calculated orientation of the Dy magnetic moment in $\left[C p^{\text {tet }}{ }_{2} \mathrm{Dy}\left(\eta^{2}-\mathrm{dbCOT}\right)\right]^{-}, \mathbf{2}$, under consideration of the charge delocalization schemes listed in Table S7. (A) Model A (coordinating carbon atoms carry negative charge only); (B) Model B (negative charge delocalized on COT $\left(\mathrm{C}_{8}\right)$ ring only); $(\mathbf{C})$ Model $\mathrm{C}$ (uniform charge distribution over the dbCOT ligand); (D) NBO charges, (E) Mulliken charges. 


\subsection{Computational Data}

Table S8. Comparison of experimental and computed bond metrics for the anion $\left[\mathrm{Cp}_{2}^{\text {tet }} \mathrm{Y}(\mathrm{dbCOT})\right]$ in 1, using the B3LYP functional with varying basis sets.

\begin{tabular}{|c|c|c|c|c|}
\hline $\mathbf{C}_{\mathrm{dbCOT}}-\mathrm{C}_{\mathrm{dbCOT}}$ & Experimental & $\begin{array}{l}\text { def2-SV(P) } \\
6-31 G(d, p)\end{array}$ & $\begin{array}{l}\text { def2- } \\
\text { SV(P) }\end{array}$ & $\begin{array}{c}\text { def2-SV(P) } \\
6-31+G(d, p)\end{array}$ \\
\hline \multicolumn{5}{|c|}{ Distances $(\AA)$} \\
\hline$C^{2}-C^{3}$ & 1.424 & 1.435 & 1.435 & 1.435 \\
\hline$C^{1}-C^{2}$ & 1.431 & 1.433 & 1.433 & 1.434 \\
\hline$C^{1}-C^{16}$ & 1.426 & 1.426 & 1.428 & 1.428 \\
\hline$C^{11}-C^{16}$ & 1.464 & 1.462 & 1.463 & 1.461 \\
\hline$C^{115}-C^{16}$ & 1.435 & 1.449 & 1.45 & 1.45 \\
\hline$C^{14}-C^{15}$ & 1.358 & 1.382 & 1.383 & 1.382 \\
\hline$C^{13}-C^{14}$ & 1.405 & 1.408 & 1.411 & 1.412 \\
\hline$C^{12}-C^{13}$ & 1.382 & 1.385 & 1.386 & 1.386 \\
\hline$C^{11}-C^{12}$ & 1.42 & 1.422 & 1.425 & 1.424 \\
\hline$C^{10}-C^{11}$ & 1.421 & 1.431 & 1.433 & 1.432 \\
\hline$C^{9}-C^{10}$ & 1.379 & 1.382 & 1.385 & 1.385 \\
\hline$C^{8}-C^{9}$ & 1.438 & 1.438 & 1.439 & 1.439 \\
\hline$C^{3}-C^{8}$ & 1.438 & 1.445 & 1.448 & 1.446 \\
\hline$C^{7}-C^{8}$ & 1.429 & 1.434 & 1.436 & 1.435 \\
\hline$C^{11}-C^{16}$ & 1.37 & 1.377 & 1.379 & 1.379 \\
\hline$c^{5}-c^{6}$ & 1.395 & 1.415 & 1.417 & 1.418 \\
\hline$C^{4}-C^{5}$ & 1.353 & 1.373 & 1.376 & 1.375 \\
\hline \multirow[t]{5}{*}{$C^{3}-c^{4}$} & 1.445 & 1.437 & 1.439 & 1.438 \\
\hline & $\mathrm{MD}$ & 0.007 & 0.009 & 0.008 \\
\hline & MSE & 1.122E-04 & 1.398E-04 & 1.348E-04 \\
\hline & RMSE & 0.011 & 0.012 & 0.012 \\
\hline & MAPE & $0.561 \%$ & $0.664 \%$ & $0.660 \%$ \\
\hline $\begin{array}{c}-C_{d b c o t} \\
\text { and } \\
Y-C_{C p}\end{array}$ & Experimental & $\begin{array}{l}\text { def2-SV(P) } \\
6-31 G(d, p)\end{array}$ & $\begin{array}{l}\text { def2- } \\
\text { SV(P) }\end{array}$ & $\begin{array}{c}\text { def2-SV(P) } \\
6-31+G(d, p)\end{array}$ \\
\hline \multicolumn{5}{|c|}{ Distances $(\AA)$} \\
\hline$Y-C^{2}$ & 2.567 & 2.619 & 2.621 & 2.628 \\
\hline$Y-C^{1}$ & 2.467 & 2.48 & 2.475 & 2.496 \\
\hline$Y-C^{16}$ & 2.897 & 2.765 & 2.793 & 2.818 \\
\hline$Y-C^{3}$ & 3.444 & 3.475 & 3.483 & 3.482 \\
\hline \multirow{5}{*}{$Y-C_{c_{p}}(A v g)$} & 2.6712 & 2.7175 & 2.7117 & 2.7233 \\
\hline & $\mathrm{MD}$ & 0.002 & 0.008 & 0.020 \\
\hline & MSE & 0.005 & 0.003 & 0.003 \\
\hline & RMSE & 0.068 & 0.058 & 0.055 \\
\hline & MAPE & $1.949 \%$ & $1.733 \%$ & $1.867 \%$ \\
\hline
\end{tabular}


Table S9. Comparison of experimental and computed bond metrics for the $\left[\mathrm{Cp}^{\text {tet }}{ }_{2} \mathrm{Y}\left(\eta^{2}-\mathrm{dbCOT}\right)\right]$ anion in 1, using the PBE functional with varying basis sets.

\begin{tabular}{|c|c|c|c|c|}
\hline $\mathrm{C}_{\mathrm{dbcOT}}-\mathrm{C}_{\mathrm{dbCOT}}$ & Experimental & $\begin{array}{l}\text { def2-SV(P) } \\
\text { 6-31G(d,p) }\end{array}$ & $\begin{array}{l}\text { def2- } \\
\text { SV(P) }\end{array}$ & $\begin{array}{c}\text { def2-SV(P) } \\
6-31+G(d, p)\end{array}$ \\
\hline \multicolumn{5}{|c|}{ Distances $(\AA)$} \\
\hline$C^{2}-C^{3}$ & 1.424 & 1.438 & 1.439 & 1.439 \\
\hline$c^{1}-c^{2}$ & 1.431 & 1.434 & 1.434 & 1.435 \\
\hline$C^{1}-C^{16}$ & 1.426 & 1.432 & 1.433 & 1.434 \\
\hline$C^{11}-C^{16}$ & 1.464 & 1.468 & 1.469 & 1.468 \\
\hline$C^{115}-C^{16}$ & 1.435 & 1.451 & 1.452 & 1.452 \\
\hline$C^{14}-C^{15}$ & 1.358 & 1.392 & 1.394 & 1.392 \\
\hline$C^{13}-C^{14}$ & 1.405 & 1.412 & 1.414 & 1.415 \\
\hline$C^{12}-C^{13}$ & 1.382 & 1.395 & 1.396 & 1.396 \\
\hline$C^{11}-C^{12}$ & 1.42 & 1.425 & 1.428 & 1.428 \\
\hline$C^{10}-C^{11}$ & 1.421 & 1.433 & 1.435 & 1.435 \\
\hline$C^{9}-C^{10}$ & 1.379 & 1.391 & 1.394 & 1.394 \\
\hline$C^{8}-C^{9}$ & 1.438 & 1.441 & 1.442 & 1.441 \\
\hline$C^{3}-C^{8}$ & 1.438 & 1.455 & 1.457 & 1.455 \\
\hline$C^{7}-C^{8}$ & 1.429 & 1.438 & 1.44 & 1.44 \\
\hline$C^{11}-C^{16}$ & 1.37 & 1.385 & 1.387 & 1.387 \\
\hline$c^{5}-c^{6}$ & 1.395 & 1.419 & 1.421 & 1.422 \\
\hline$C^{4}-c^{5}$ & 1.353 & 1.383 & 1.385 & 1.384 \\
\hline \multirow[t]{5}{*}{$C^{3}-c^{4}$} & 1.445 & 1.439 & 1.441 & 1.44 \\
\hline & $\mathrm{MD}$ & 0.012 & 0.014 & 0.014 \\
\hline & MSE & 2.398E-04 & 2.866E-04 & $2.761 \mathrm{E}-04$ \\
\hline & RMSE & 0.015 & 0.017 & 0.017 \\
\hline & MAPE & $0.916 \%$ & $1.019 \%$ & $1.011 \%$ \\
\hline $\begin{array}{c}-C_{d b c o t} \\
\text { and } \\
Y-C_{C p}\end{array}$ & Experimental & $\begin{array}{l}\text { def2-SV(P) } \\
\text { 6-31G(d,p) }\end{array}$ & $\begin{array}{l}\text { def2- } \\
\text { SV(P) }\end{array}$ & $\begin{array}{c}\text { def2-SV(P) } \\
6-31+G(d, p)\end{array}$ \\
\hline \multicolumn{5}{|c|}{ Distances $(\AA)$} \\
\hline$Y-C^{2}$ & 2.567 & 2.659 & 2.666 & 2.665 \\
\hline$Y-C^{1}$ & 2.467 & 2.471 & 2.464 & 2.488 \\
\hline$Y-C^{16}$ & 2.897 & 2.733 & 2.749 & 2.777 \\
\hline$Y-C^{3}$ & 3.444 & 3.557 & 3.575 & 3.551 \\
\hline \multirow[t]{5}{*}{$Y-C_{c_{p}}(A v g)$} & 2.6712 & 2.7063 & 2.7014 & 2.7137 \\
\hline & $M D$ & 0.016 & 0.022 & 0.030 \\
\hline & MSE & 0.010 & 0.010 & 0.008 \\
\hline & RMSE & 0.099 & 0.100 & 0.087 \\
\hline & MAPE & $2.800 \%$ & $2.804 \%$ & $2.702 \%$ \\
\hline
\end{tabular}


Table S10. Comparison of experimental and computed bond metrics for the $\left[\mathrm{Cp}^{\text {tet }}{ }_{2} \mathrm{Y}\left(\eta^{2}-\right.\right.$ $\mathrm{dbCOT})$ ] anion in 1, using the PBE0 functional with varying basis sets.

\begin{tabular}{|c|c|c|c|c|}
\hline$C_{d b C O T}-C_{d b C O T}$ & Experimental & $\begin{array}{l}\text { def2-SV(P) } \\
\text { 6-31G(d,p) }\end{array}$ & $\begin{array}{l}\text { def2- } \\
\text { SV(P) }\end{array}$ & $\begin{array}{c}\text { def2-SV(P) } \\
6-31+G(d, p) \\
\end{array}$ \\
\hline \multicolumn{5}{|c|}{ Distances $(\AA)$} \\
\hline$C^{2}-C^{3}$ & 1.424 & 1.429 & 1.429 & 1.429 \\
\hline$C^{1}-C^{2}$ & 1.431 & 1.428 & 1.428 & 1.428 \\
\hline$C^{1}-C^{16}$ & 1.426 & 1.421 & 1.422 & 1.423 \\
\hline$C^{11}-C^{16}$ & 1.464 & 1.456 & 1.457 & 1.455 \\
\hline$C^{115}-C^{16}$ & 1.435 & 1.444 & 1.445 & 1.445 \\
\hline$C^{14}-C^{15}$ & 1.358 & 1.38 & 1.38 & 1.379 \\
\hline$C^{13}-C^{14}$ & 1.405 & 1.404 & 1.407 & 1.408 \\
\hline$C^{12}-C^{13}$ & 1.382 & 1.383 & 1.384 & 1.383 \\
\hline$C^{11}-C^{12}$ & 1.42 & 1.416 & 1.419 & 1.419 \\
\hline$C^{10}-C^{11}$ & 1.421 & 1.427 & 1.428 & 1.428 \\
\hline$C^{9}-c^{10}$ & 1.379 & 1.378 & 1.381 & 1.381 \\
\hline$C^{8}-C^{9}$ & 1.438 & 1.434 & 1.435 & 1.434 \\
\hline$C^{3}-c^{8}$ & 1.438 & 1.441 & 1.444 & 1.441 \\
\hline$C^{7}-C^{8}$ & 1.429 & 1.429 & 1.431 & 1.43 \\
\hline$C^{11}-C^{16}$ & 1.37 & 1.373 & 1.375 & 1.375 \\
\hline$C^{5}-C^{6}$ & 1.395 & 1.412 & 1.414 & 1.414 \\
\hline$C^{4}-C^{5}$ & 1.353 & 1.37 & 1.372 & 1.372 \\
\hline \multirow[t]{5}{*}{$c^{3}-c^{4}$} & 1.445 & 1.432 & 1.434 & 1.433 \\
\hline & $\mathrm{MD}$ & 0.002 & 0.004 & 0.004 \\
\hline & MSE & 8.467E-05 & 9.233E-05 & 9.144E-05 \\
\hline & RMSE & 0.009 & 0.010 & 0.010 \\
\hline & MAPE & $0.484 \%$ & $0.516 \%$ & $0.508 \%$ \\
\hline $\begin{array}{c}\mathrm{Y}-\mathbf{C}_{\mathrm{dbcOT}} \\
\text { and } \\
\mathrm{Y}-\mathrm{C}_{\mathrm{cp}}\end{array}$ & Experimental & $\begin{array}{l}\text { def2-SV(P) } \\
\text { 6-31G(d,p) }\end{array}$ & $\begin{array}{l}\text { def2- } \\
\text { SV(P) }\end{array}$ & $\begin{array}{c}\text { def2-SV(P) } \\
6-31+G(d, p)\end{array}$ \\
\hline \multicolumn{5}{|c|}{ Distances $(\AA)$} \\
\hline$Y-C^{2}$ & 2.567 & 2.616 & 2.618 & 2.621 \\
\hline$Y-C^{1}$ & 2.467 & 2.456 & 2.451 & 2.473 \\
\hline$Y-C^{16}$ & 2.897 & 2.728 & 2.756 & 2.775 \\
\hline$Y-C^{3}$ & 3.444 & 3.486 & 3.495 & 3.48 \\
\hline \multirow[t]{5}{*}{$Y-C_{c_{p}}(A v g)$} & 2.6712 & 2.6917 & 2.6867 & 2.6972 \\
\hline & MD & -0.014 & -0.008 & 0.000 \\
\hline & MSE & 0.007 & 0.005 & 0.004 \\
\hline & RMSE & 0.082 & 0.072 & 0.063 \\
\hline & MAPE & $2.035 \%$ & $1.913 \%$ & $1.715 \%$ \\
\hline
\end{tabular}


Table S11. Comparison of experimental and computed bond metrics for the $\left[\mathrm{Cp}^{\text {tet }}{ }_{2} \mathrm{Y}\left(\eta^{2}-\mathrm{dbCOT}\right)\right]$ anion in 1, using the TPSS functional with varying basis sets.

\begin{tabular}{|c|c|c|c|c|}
\hline $\mathbf{C}_{\mathrm{dbCOT}}-\mathbf{C}_{\mathrm{dbCOT}}$ & Experimental & $\begin{array}{l}\text { def2-SV(P) } \\
6-31 G(d, p)\end{array}$ & $\begin{array}{l}\text { def2- } \\
\text { SV(P) } \\
\end{array}$ & $\begin{array}{c}\text { def2-SV(P) } \\
6-31+G(d, p)\end{array}$ \\
\hline \multicolumn{5}{|c|}{ Distances $(\AA)$} \\
\hline $\mathrm{C}^{2}-\mathrm{C}^{3}$ & 1.424 & 1.439 & 1.439 & 1.433 \\
\hline$c^{1}-c^{2}$ & 1.431 & 1.436 & 1.435 & 1.439 \\
\hline$C^{1}-C^{16}$ & 1.426 & 1.431 & 1.432 & 1.452 \\
\hline$C^{11}-C^{16}$ & 1.464 & 1.467 & 1.468 & 1.509 \\
\hline$C^{115}-C^{16}$ & 1.435 & 1.452 & 1.452 & 1.467 \\
\hline$C^{14}-C^{15}$ & 1.358 & 1.392 & 1.393 & 1.439 \\
\hline$C^{13}-C^{14}$ & 1.405 & 1.41 & 1.412 & 1.43 \\
\hline$C^{12}-C^{13}$ & 1.382 & 1.395 & 1.397 & 1.413 \\
\hline$C^{11}-C^{12}$ & 1.42 & 1.423 & 1.425 & 1.392 \\
\hline$C^{10}-C^{11}$ & 1.421 & 1.433 & 1.434 & 1.434 \\
\hline$C^{9}-C^{10}$ & 1.379 & 1.39 & 1.392 & 1.396 \\
\hline$C^{8}-C^{9}$ & 1.438 & 1.442 & 1.442 & 1.442 \\
\hline$C^{3}-C^{8}$ & 1.438 & 1.453 & 1.455 & 1.429 \\
\hline$C^{7}-C^{8}$ & 1.429 & 1.438 & 1.439 & 1.383 \\
\hline$C^{11}-C^{16}$ & 1.37 & 1.384 & 1.385 & 1.422 \\
\hline$C^{5}-c^{6}$ & 1.395 & 1.419 & 1.421 & 1.429 \\
\hline$c^{4}-c^{5}$ & 1.353 & 1.381 & 1.383 & 1.392 \\
\hline \multirow[t]{5}{*}{$C^{3}-c^{4}$} & 1.445 & 1.438 & 1.44 & 1.453 \\
\hline & $\overline{M D}$ & 0.012 & 0.013 & 0.019 \\
\hline & MSE & 2.291E-04 & 2.597E-04 & 1.162E-03 \\
\hline & RMSE & 0.015 & 0.016 & 0.034 \\
\hline & MAPE & $0.891 \%$ & $0.959 \%$ & $2.012 \%$ \\
\hline $\begin{array}{c}\mathrm{Y}-\mathrm{C}_{\mathrm{dbcot}} \\
\text { and } \\
\mathrm{Y}-\mathrm{C}_{\mathrm{cp}}\end{array}$ & Experimental & $\begin{array}{l}\text { def2-SV(P) } \\
6-31 G(d, p)\end{array}$ & $\begin{array}{l}\text { def2- } \\
\text { SV(P) }\end{array}$ & $\begin{array}{c}\text { def2-SV(P) } \\
6-31+G(d, p)\end{array}$ \\
\hline \multicolumn{5}{|c|}{ Distances $(\AA)$} \\
\hline$Y-C^{2}$ & 2.567 & 2.656 & 2.669 & 2.662 \\
\hline$Y-C^{1}$ & 2.467 & 2.472 & 2.465 & 2.489 \\
\hline$Y-C^{16}$ & 2.897 & 2.707 & 2.719 & 2.746 \\
\hline$Y-C^{3}$ & 3.444 & 3.55 & 3.575 & 2.995 \\
\hline \multirow[t]{5}{*}{$Y-C_{C_{p}}(A v g)$} & 2.6712 & 2.6963 & 2.693 & 2.7023 \\
\hline & $\mathrm{MD}$ & 0.007 & 0.015 & -0.090 \\
\hline & MSE & 0.011 & 0.012 & 0.047 \\
\hline & RMSE & 0.106 & 0.109 & 0.217 \\
\hline & MAPE & $2.849 \%$ & $2.964 \%$ & $4.801 \%$ \\
\hline
\end{tabular}


Table S12. Comparison of experimental and computed bond metrics for the $\left[\mathrm{Cp}^{\text {tet }}{ }_{2} \mathrm{Y}\left(\eta^{2}-\mathrm{dbCOT}\right)\right]$ anion in 1, using the TPSSh functional with varying basis sets.

\begin{tabular}{|c|c|c|c|c|}
\hline $\mathrm{C}_{\mathrm{dbCOT}}-\mathrm{C}_{\mathrm{dbCOT}}$ & Experimental & $\begin{array}{l}\text { def2-SV(P) } \\
\text { 6-31G(d,p) }\end{array}$ & $\begin{array}{l}\text { def2- } \\
\text { SV(P) }\end{array}$ & $\begin{array}{c}\text { def2-SV(P) } \\
6-31+G(d, p) \\
\end{array}$ \\
\hline \multicolumn{5}{|c|}{ Distances $(\AA)$} \\
\hline$C^{2}-C^{3}$ & 1.424 & 1.435 & 1.435 & 1.435 \\
\hline$c^{1}-c^{2}$ & 1.431 & 1.433 & 1.432 & 1.433 \\
\hline$C^{1}-C^{16}$ & 1.426 & 1.426 & 1.427 & 1.428 \\
\hline$C^{11}-C^{16}$ & 1.464 & 1.462 & 1.464 & 1.462 \\
\hline$C^{115}-C^{16}$ & 1.435 & 1.448 & 1.449 & 1.449 \\
\hline$C^{14}-C^{15}$ & 1.358 & 1.387 & 1.388 & 1.386 \\
\hline$C^{13}-C^{14}$ & 1.405 & 1.407 & 1.409 & 1.411 \\
\hline$C^{12}-C^{13}$ & 1.382 & 1.39 & 1.391 & 1.39 \\
\hline$C^{11}-C^{12}$ & 1.42 & 1.419 & 1.422 & 1.422 \\
\hline$C^{10}-C^{11}$ & 1.421 & 1.431 & 1.432 & 1.432 \\
\hline$C^{9}-C^{10}$ & 1.379 & 1.384 & 1.387 & 1.387 \\
\hline$C^{8}-C^{9}$ & 1.438 & 1.439 & 1.439 & 1.439 \\
\hline$C^{3}-C^{8}$ & 1.438 & 1.447 & 1.45 & 1.448 \\
\hline$C^{7}-C^{8}$ & 1.429 & 1.434 & 1.436 & 1.435 \\
\hline$C^{11}-C^{16}$ & 1.37 & 1.379 & 1.381 & 1.381 \\
\hline$c^{5}-c^{6}$ & 1.395 & 1.416 & 1.418 & 1.418 \\
\hline$C^{4}-c^{5}$ & 1.353 & 1.376 & 1.378 & 1.378 \\
\hline \multirow[t]{5}{*}{$C^{3}-C^{4}$} & 1.445 & 1.435 & 1.437 & 1.437 \\
\hline & MD & 0.007 & 0.009 & 0.009 \\
\hline & MSE & 1.439E-04 & $1.688 \mathrm{E}-04$ & 1.599E-04 \\
\hline & RMSE & 0.012 & 0.013 & 0.013 \\
\hline & MAPE & $0.642 \%$ & $0.710 \%$ & $0.709 \%$ \\
\hline $\begin{array}{c}-C_{d b c O T} \\
\text { and } \\
Y-C_{c p}\end{array}$ & Experimental & $\begin{array}{l}\text { def2-SV(P) } \\
\text { 6-31G(d,p) }\end{array}$ & $\begin{array}{l}\text { def2- } \\
\text { SV(P) }\end{array}$ & $\begin{array}{c}\text { def2-SV(P) } \\
6-31+G(d, p)\end{array}$ \\
\hline \multicolumn{5}{|c|}{ Distances $(\AA)$} \\
\hline$Y-C^{2}$ & 2.567 & 2.639 & 2.65 & 2.645 \\
\hline$Y-C^{1}$ & 2.467 & 2.466 & 2.46 & 2.483 \\
\hline$Y-C^{16}$ & 2.897 & 2.706 & 2.721 & 2.746 \\
\hline$Y-C^{3}$ & 3.444 & 3.523 & 3.543 & 3.515 \\
\hline \multirow[t]{5}{*}{$Y-C_{c_{p}}(A v g)$} & 2.6712 & 2.6925 & 2.689 & 2.698 \\
\hline & $\mathrm{MD}$ & -0.004 & 0.003 & 0.008 \\
\hline & MSE & 0.010 & 0.010 & 0.007 \\
\hline & RMSE & 0.098 & 0.098 & 0.084 \\
\hline & MAPE & $2.506 \%$ & $2.627 \%$ & $2.393 \%$ \\
\hline
\end{tabular}




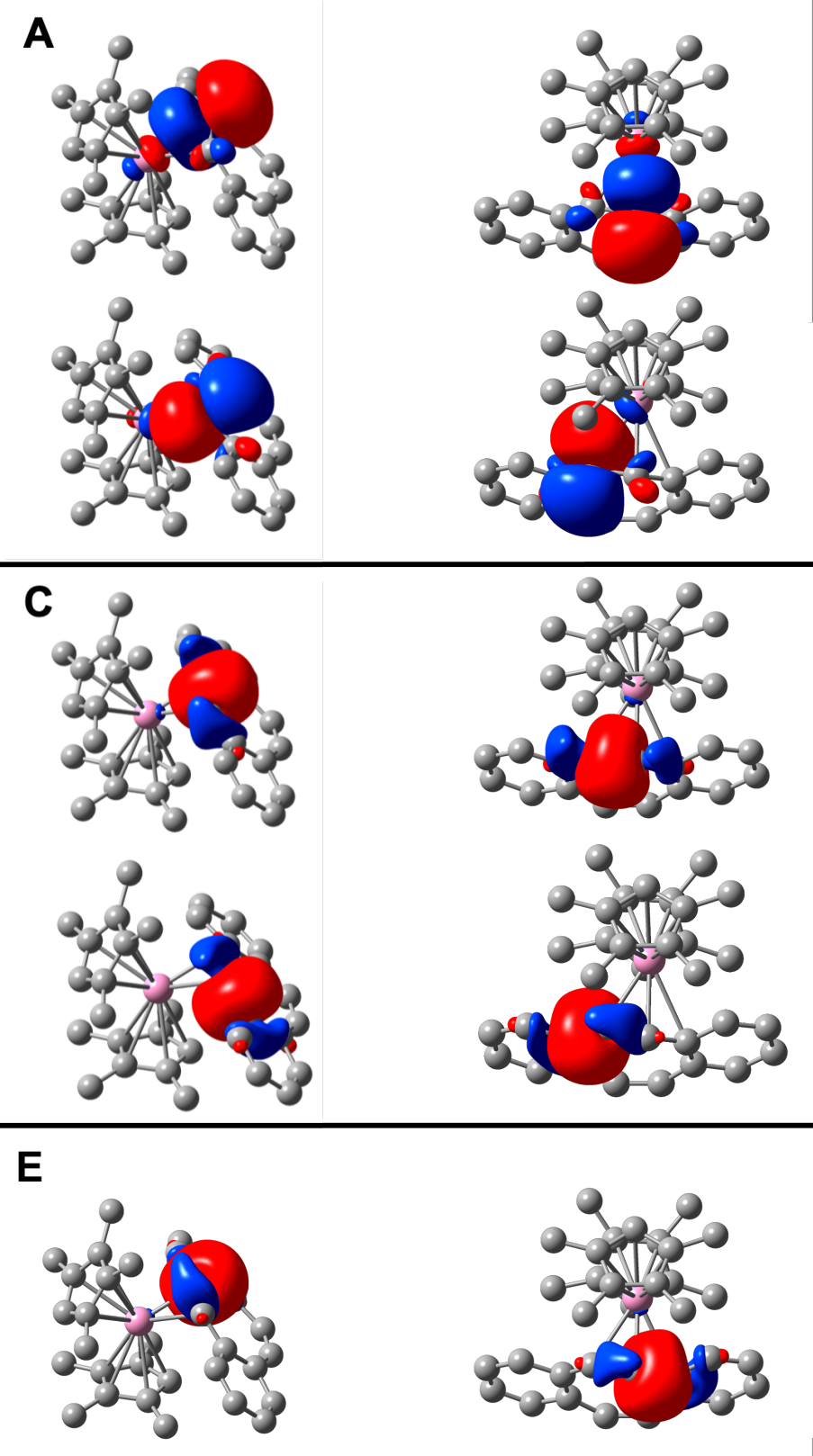

B
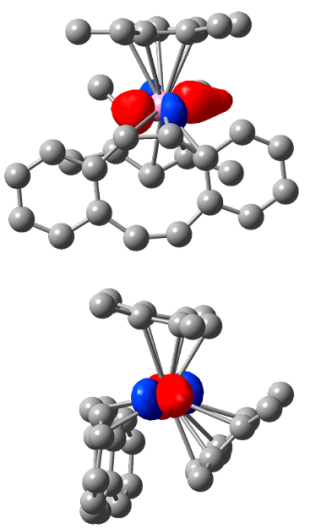

D
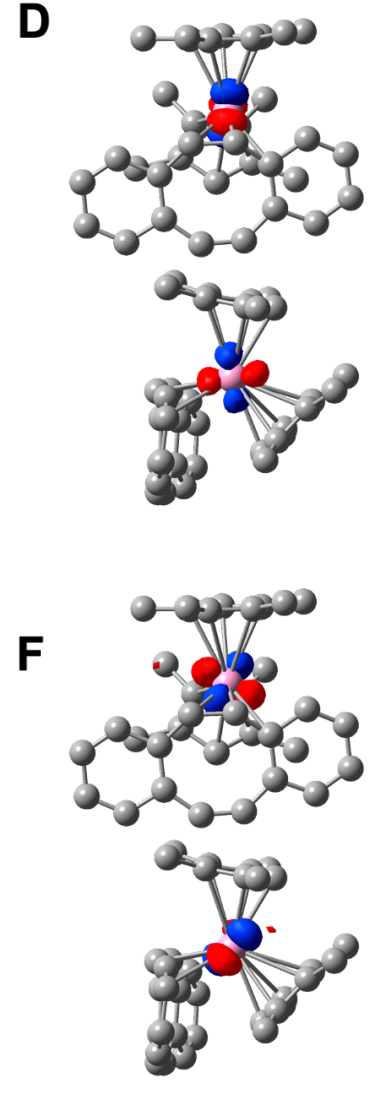

Figure S36. Calculated Natural Bond Orbitals (NBOs) of the $\left[\mathrm{Cp}^{\text {tet }}{ }_{2} \mathrm{Y}\left(\mathrm{n}^{2}-\mathrm{dbCOT}\right)\right]^{-}$anion in 1, with the PBE0 functional and def2-TZVP\&6-311G(d,p) basis sets. (A) NBOs of the lone pairs on the $\mathrm{C}^{1}$ (top) and $\mathrm{C}^{2}$ (bottom) atoms from the side and top perspective. (B) Relevant vacant acceptor NBO on $Y$ for the $C^{1}$ and $C^{2}$ donor lone pair NBOs from the front and side perspective. (C) NBOs of the $C^{1}-C^{2}$ bond (top) and $C^{2}-C^{3}$ bond (bottom) from the side and top perspective. (D) Relevant vacant acceptor NBO on $Y$ for the donor NBOs from the $C^{1}-C^{2}$ and $C^{2}-C^{3}$ bonds from the front and side perspective. (E) NBO of the $C^{1}-C^{16}$ bond from the side and top perspective. (F) Relevant vacant acceptor NBO on $\mathrm{Y}$ for the donor lone pair NBO of the $\mathrm{C}^{1}-\mathrm{C}^{16}$ bond from the front and side perspective. Isovalues for the carbon- and yttrium-based NBOs were set to 0.02 and 0.08 , respectively. 

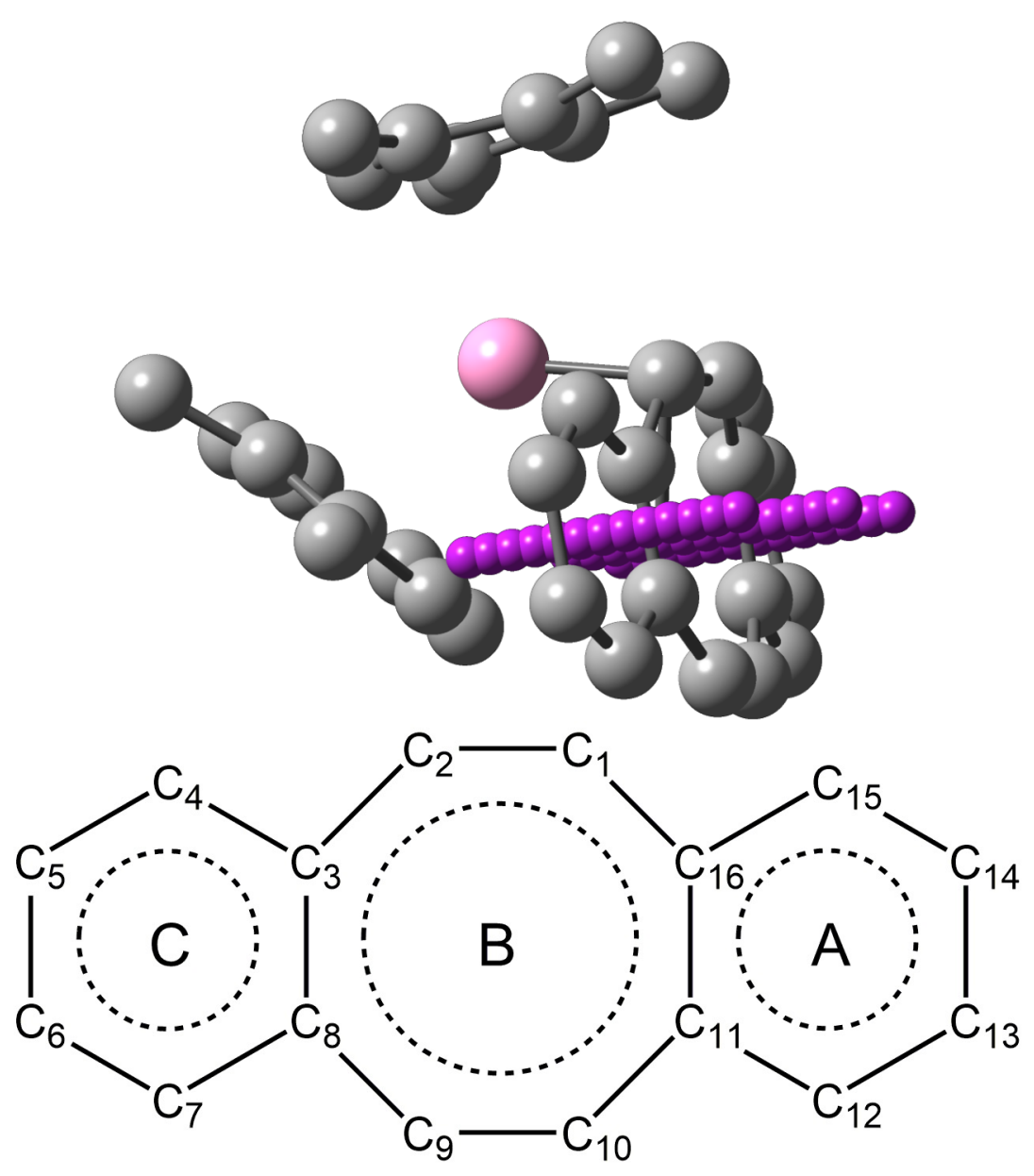

Figure S37. Nucleus independent chemical shift values for the $\left[\mathrm{Cp}^{\text {tet }}{ }_{2} \mathrm{Y}\left(\eta^{2}-\mathrm{dbCOT}\right)\right]^{-}$anion in 1, were computed at the centroid of rings $A, B$, and $C$, as well as $\pm 1.5 \AA$ perpendicular to the ring plane in $0.25 \AA$ intervals. The pink spheres represent the points at which the magnetic shielding tensors were calculated (top). Labeling scheme for the rings of the (dbCOT) ${ }^{2-}$ moiety (bottom). 
Table S13. Calculated NICS values for rings $A, B$, and $C \pm 1.5 \AA$ perpendicular to the ring plane in $0.25 \AA$ intervals using the PBE0 functional and def2-TZVP\&6-311G(d,p) basis sets.

\begin{tabular}{c|ccc}
\hline $\begin{array}{c}\text { Distance from } \\
\text { Centroid }\end{array}$ & $\begin{array}{c}\text { Chemical Shift } \\
\text { Ring A }\end{array}$ & $\begin{array}{c}\text { Chemical Shift } \\
\text { Ring B }\end{array}$ & $\begin{array}{c}\text { Chemical Shift } \\
\text { Ring C }\end{array}$ \\
\hline$(\AA)$ & & $(\mathrm{ppm})$ & -19.1 \\
\hline+1.50 & -20.5 & -26.0 & -20.4 \\
+1.25 & -22.0 & -27.7 & -20.3 \\
+1.00 & -22.1 & -28.3 & -18.1 \\
+0.75 & -19.7 & -27.7 & -13.8 \\
+0.50 & -15.1 & -26.3 & -9.3 \\
+0.25 & -10.4 & -24.9 & -8.2 \\
0.00 & -7.7 & -12.1 & -10.6 \\
-0.25 & -12.9 & -26.4 & -16.5 \\
-0.50 & -19.9 & -29.2 & -22.2 \\
-0.75 & -26.3 & -32.1 & -25.4 \\
-1.00 & -30.0 & -34.2 & -25.8 \\
-1.25 & -31.0 & -35.1 & -24.4 \\
-1.50 & -30.5 & -35.2 & \\
\hline
\end{tabular}

* See Figure S37 for ring labeling. 


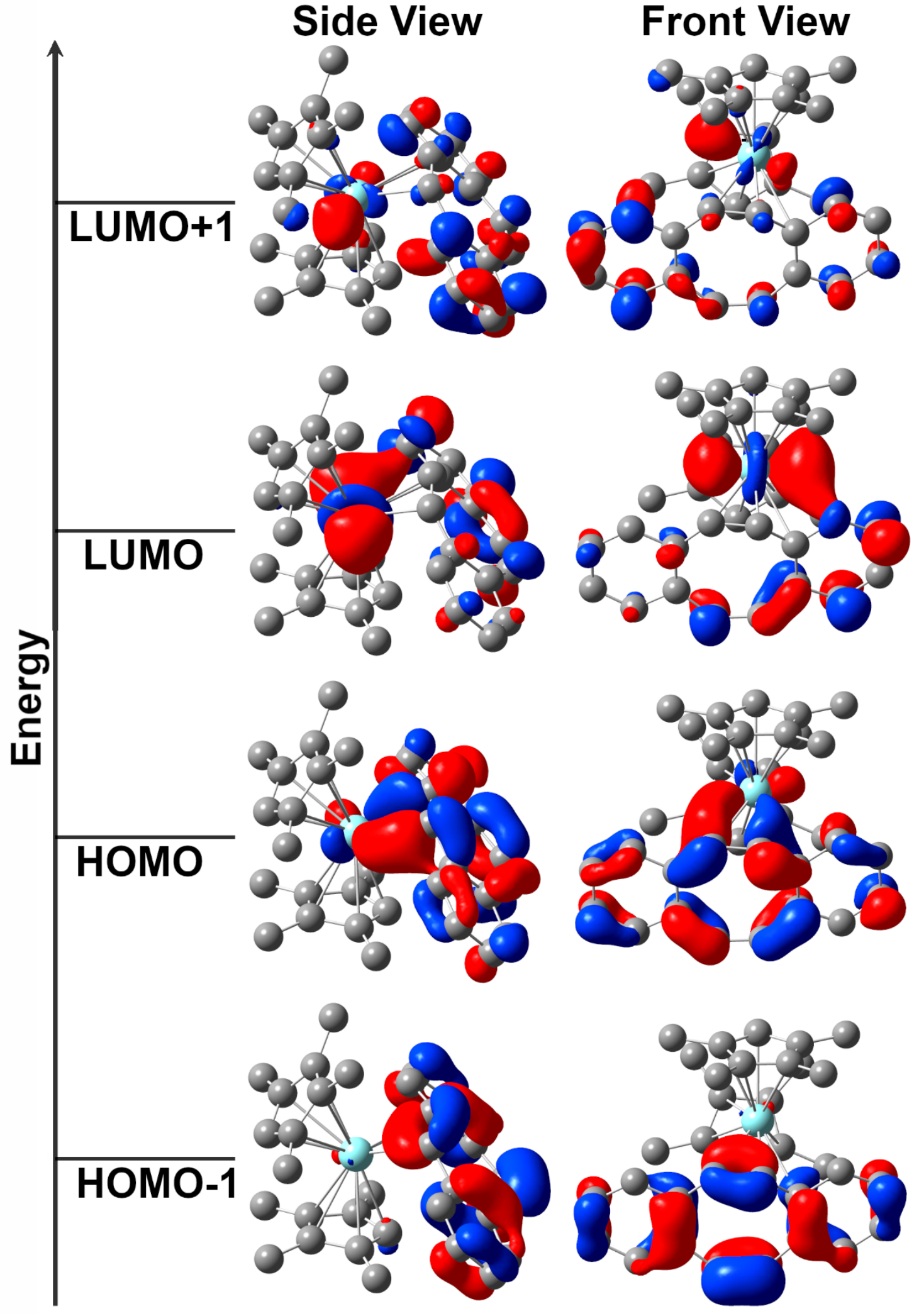

Figure S38. Calculated frontier orbitals for the $\left[\mathrm{Cp}^{\text {tet }}{ }_{2} \mathrm{Y}\left(\eta^{2}-\mathrm{dbCOT}\right)\right]^{-}$anion in 1, with the PBE0 functional and def2-TZVP\&6-311G(d,p) basis sets. All isovalues were set to 0.04 . 
Table S14. Calculated transitions of $\left[\mathrm{Cp}^{\text {tet }}{ }_{2} \mathrm{Y}\left(\eta^{2}-\mathrm{dbCOT}\right)\right]^{-}$anion in 1 from time-dependent density functional theory calculations (TD-DFT), using the PBE0 functional and def2-TZVP\&6-311G(d,p) basis sets with solvent considerations (SMD, THF).

\begin{tabular}{|c|c|c|c|c|}
\hline & $\mathrm{cm}^{-1}$ & $\begin{array}{c}\text { Oscillator } \\
\text { Strength }\end{array}$ & Molecular Orbitals & Assignment \\
\hline 1 & 16044 & 0.1183 & HOMO - LUMO & LMCT \\
\hline 2 & 23111 & 0.1431 & HOMO - LUMO+2 & LMCT \\
\hline 3 & 24779 & 0.1284 & HOMO - LUMO+3 & LMCT \\
\hline 4 & 27123 & 0.5831 & HOMO-1 - LUMO & LMCT \\
\hline 5 & 28253 & 0.2224 & $\mathrm{HOMO}-\mathrm{LUMO}+4$ & LMCT \\
\hline 6 & 29937 & 0.2538 & HOMO - LUMO+5 & LMCT \\
\hline 7 & 32402 & 0.475 & HOMO-1 - LUMO+1 & $\begin{array}{l}\text { Interligand CT } \\
\left(C p^{\text {tet }}-d b C O T\right)\end{array}$ \\
\hline 8 & 33064 & 0.0728 & HOMO-3 - LUMO & $\begin{array}{c}\text { Intralingand } \mathrm{CT} \\
(\mathrm{dbCOT})\end{array}$ \\
\hline 9 & 34046 & 0.2102 & HOMO-1 - LUMO+2 & $\begin{array}{l}\text { Interligand CT } \\
\left(C p^{\text {tet }}-d b C O T\right)\end{array}$ \\
\hline 10 & 34767 & 0.0817 & HOMO - LUMO+10 & $\begin{array}{c}\text { Intraligand } C T \\
(\mathrm{dbCOT})\end{array}$ \\
\hline 11 & 35524 & 0.1727 & HOMO-1 - LUMO+2 & LMCT \\
\hline 12 & 38438 & 0.0901 & HOMO-5 - LUMO & $\begin{array}{l}\text { Interligand CT } \\
\left(\mathrm{Cp}^{\text {tet }}-\mathrm{dbCOT}\right)\end{array}$ \\
\hline 13 & 38653 & 0.0438 & HOMO-2 - LUMO+1 & $\begin{array}{l}\text { Interligand CT } \\
\left(\mathrm{Cp}^{\text {tet }}-\mathrm{dbCOT}\right)\end{array}$ \\
\hline 14 & 38852 & 0.0504 & HOMO-1 -LUMO+3 & LMCT \\
\hline 15 & 39559 & 0.0691 & HOMO-2 - LUMO+1 & $\begin{array}{l}\text { Interligand CT } \\
\left(\mathrm{Cp}^{\text {tet }}-\mathrm{dbCOT}\right)\end{array}$ \\
\hline
\end{tabular}


Table S15. Calculated bond metrics from the optimized geometries of the temperature-dependent single-crystal X-ray measurements on $\left[K\left(\right.\right.$ crypt-222)][C $\left.p^{\text {tet }}{ }_{2} \mathrm{Y}\left(\eta^{2}-\mathrm{dbCOT}\right)\right](\mathbf{1})$, using the PBE0 functional and def2-TZVP\&6-311G(d,p) basis sets.

\begin{tabular}{|c|c|c|c|c|c|c|}
\hline Atoms & $100 \mathrm{~K}$ & 135K & $170 \mathrm{~K}$ & 205K & 240K & $298 \mathrm{~K}$ \\
\hline & \multicolumn{6}{|c|}{ Y-dbCOT distances $(\AA)$} \\
\hline $\mathrm{Y} 1-\mathrm{C} 1$ & 2.4603 & 2.6184 & 2.6184 & 2.4603 & 2.6185 & 2.6185 \\
\hline Y1-C2 & 2.7003 & 2.4603 & 2.4603 & 2.6184 & 2.4603 & 2.4603 \\
\hline Y1-C3 & 3.4776 & 2.7526 & 2.7526 & 3.4775 & 2.7523 & 2.7523 \\
\hline \multirow[t]{2}{*}{$\mathrm{Y} 1-\mathrm{C} 16$} & 2.7523 & 3.4775 & 3.4775 & 2.7526 & 3.4772 & 3.4772 \\
\hline & \multicolumn{6}{|c|}{$Y-C p^{\text {tet }}$ distances (Top) $(\AA)$} \\
\hline $\mathrm{Y} 1-\mathrm{C} 26$ & 2.6166 & 2.6167 & 2.6166 & 2.6167 & 2.6167 & 2.6167 \\
\hline Y1-C27 & 2.6466 & 2.6752 & 2.6752 & 2.6464 & 2.6465 & 2.6751 \\
\hline Y1-C28 & 2.7003 & 2.7208 & 2.7208 & 2.6999 & 2.7002 & 2.721 \\
\hline $\mathrm{Y} 1-\mathrm{C} 29$ & 2.7208 & 2.6999 & 2.6999 & 2.7208 & 2.721 & 2.7002 \\
\hline \multirow[t]{2}{*}{$\mathrm{Y} 1-\mathrm{C} 30$} & 2.6751 & 2.6464 & 2.6464 & 2.6752 & 2.6751 & 2.6465 \\
\hline & \multicolumn{6}{|c|}{ Y-Cp ${ }^{\text {tet }}$ distances (Bottom) $(\AA)$} \\
\hline $\mathrm{Y} 1-\mathrm{C} 17$ & 2.7807 & 2.7927 & 2.7927 & 2.7811 & 2.7927 & 2.7927 \\
\hline Y1-C18 & 2.7923 & 2.7811 & 2.7811 & 2.7927 & 2.7811 & 2.7811 \\
\hline Y1-C19 & 2.6746 & 2.6618 & 2.6618 & 2.6745 & 2.6618 & 2.6618 \\
\hline Y1-C20 & 2.6018 & 2.6015 & 2.6015 & 2.6015 & 2.6016 & 2.6016 \\
\hline \multirow[t]{2}{*}{$\mathrm{Y} 1-\mathrm{C} 21$} & 2.6618 & 2.6745 & 2.6745 & 2.6618 & 2.6745 & 2.6745 \\
\hline & \multicolumn{6}{|c|}{ Distances around COT rings $(\AA)$} \\
\hline $\mathrm{C} 1-\mathrm{C} 2$ & 1.4253 & 1.4252 & 1.4253 & 1.4252 & 1.4252 & 1.4252 \\
\hline $\mathrm{C} 2-\mathrm{C} 3$ & 1.426 & 1.4191 & 1.4191 & 1.4259 & 1.4191 & 1.4191 \\
\hline C3-C4 & 1.431 & 1.4418 & 1.4418 & 1.4309 & 1.4418 & 1.4418 \\
\hline C4-C5 & 1.367 & 1.3755 & 1.3755 & 1.367 & 1.3754 & 1.3754 \\
\hline C6-C5 & 1.4107 & 1.4037 & 1.4037 & 1.4107 & 1.4036 & 1.4036 \\
\hline C7-C6 & 1.3701 & 1.3789 & 1.379 & 1.3702 & 1.379 & 1.379 \\
\hline C8-C7 & 1.428 & 1.4155 & 1.4155 & 1.428 & 1.4155 & 1.4155 \\
\hline C8-C9 & 1.4311 & 1.4243 & 1.4243 & 1.4311 & 1.4244 & 1.4244 \\
\hline C9-C10 & 1.3767 & 1.3767 & 1.3767 & 1.3768 & 1.3767 & 1.3767 \\
\hline C10-C11 & 1.4243 & 1.4311 & 1.4311 & 1.4243 & 1.431 & 1.431 \\
\hline C11-C12 & 1.4155 & 1.428 & 1.428 & 1.4155 & 1.428 & 1.428 \\
\hline $\mathrm{C} 12-\mathrm{C} 13$ & 1.379 & 1.3702 & 1.3702 & 1.3789 & 1.3702 & 1.3702 \\
\hline C13-C14 & 1.4036 & 1.4107 & 1.4107 & 1.4037 & 1.4107 & 1.4107 \\
\hline C15-C14 & 1.3754 & 1.367 & 1.3669 & 1.3755 & 1.3669 & 1.3669 \\
\hline C16-C15 & 1.4253 & 1.4252 & 1.4253 & 1.4252 & 1.4252 & 1.4252 \\
\hline $\mathrm{C} 1-\mathrm{C} 16$ & 1.426 & 1.4191 & 1.4191 & 1.4259 & 1.4191 & 1.4191 \\
\hline
\end{tabular}




\subsection{References}

(1) Evans, W. J.; Lee, D. S.; Johnston, M. A.; Ziller, J. W. Organometallics 2005, 24, 6393-6397.

(2) Bergbreiter, D. E.; Killough, J. M. J. Am. Chem. Soc. 1978, 2126-2134.

(3) Barker, B. J.; Huffman, H. L.; Sears, P. G. J. Phys. Chem. 1974, 78, 2689-2693.

(4) Bloch, J.; Kradolfer, S.; L. Gianetti, T.; Ostendorf, D.; Dey, S.; Mougel, V.; Grützmacher, H. Molecules 2020, 25, 4742.

(5) Zhu, Y.; Zhou, Z.; Wei, Z.; Petrukhina, M. A. Organometallics 2020, 39, 4688-4695.

(6) Demir, S.; Lorenz, S. E.; Fang, M.; Furche, F.; Meyer, G.; Ziller, J. W.; Evans, W. J. J. Am. Chem. Soc. 2010, 132, 11151-11158.

(7) Sheldrick, G. M. Acta Crystallogr. Sect. A 2015, 71, 3-8.

(8) Sheldrick, G. M. Acta Crystallogr. Sect. C 2015, 71, 3-8.

(9) O. V Dolomanov, L. J. Bourhis, R. J. Gildea, J. A. K. Howard; H. Puschmann J. Appl. Crystallogr. 2009, 42, 339-341.

(10) Gaussian 16, Revision B.01, Frisch, M. J., Trucks, G. W., Schlegel, H. B., Scuseria, G. E., Robb, M. A., Cheeseman, J. R., Scalmani, G., Barone, V., Petersson, G. A., Nakatsuji, H.,Li, X., Caricato, M., Marenich, A. V., Bloino, J., Janesko, B. G., Gomperts, R., Mennucci, B., Hratchian, H. P., Ortiz, J. V., Izmaylov, A. F., Sonnenberg, J. L., Williams-Young, D., Ding, F., Lipparini, F., Egidi, F., Goings, J., Peng, B., Petrone, A., Henderson, T., Ranasinghe, D., Zakrzewski, V. G., Gao, J., Rega, N., Zheng, G., Liang, W., Hada, M., Ehara, M., Toyota, K., Fukuda, R., Hasegawa, J., Ishida, M., Nakajima, T., Honda, Y., Kitao, O., Nakai, H., Vreven, T., Throssell, K., Montgomery, Jr., J. A., Peralta, J. E., Ogliaro, F., Bearpark, M. J., Heyd, J. J., Brothers, E. N., Kudin, K. N., Staroverov, V. N., Keith, T. A., Kobayashi, R., Normand, J., Raghavachari, K., Rendell, A. P., Burant, J. C., lyengar, S. S., Tomasi, J., Cossi, M.,Millam, J. M., Klene, M., Adamo, C., Cammi, R., Ochterski, J. W., Martin, R. L., Morokuma, K., Farkas, O., Foresman, J. B., Fox, D. J. Gaussian, Inc., Wallingford CT, 2016.

(11) Peterson, K. A.; Figgen, D.; Dolg, M.; Stoll, H. J. Chem. Phys. 2007, 126, 124101.

(12) Becke, A. D. J. Chem. Phys. 1993, 98, 5648-5652.

(13) Perdew, J. P.; Burke, K.; Ernzerhof, M. Phys. Rev. Lett. 1996, 77, 3865-3868.

(14) Adamo, C.; Barone, V. J. Chem. Phys. 1999, 110, 6158-6170.

(15) Tao, J.; Perdew, J. P.; Staroverov, V. N.; Scuseria, G. E. Phys. Rev. Lett. 2003, 91, 3-6.

(16) Staroverov, V. N.; Scuseria, G. E.; Tao, J.; Perdew, J. P. J. Chem. Phys. 2003, 119, 12129 12137.

(17) Waller, M. P.; Braun, H.; Hojdis, N.; Bühl, M. J. Chem. Theory Comput. 2007, 3, 2234-2242.

(18) Furche, F.; Perdew, J. P. J. Chem. Phys. 2006, 124, 1-27.

(19) Weigend, F.; Ahlrichs, R. Phys. Chem. Chem. Phys. 2005, 7, 3297-3305.

(20) Ditchfield, R.; Hehre, W. J.; Pople, J. A. J. Chem. Phys. 1971, 54, 720-723.

(21) Hariharan, P. C.; Pople, J. A. Theor. Chim. Acta 1973, 28, 213-222.

(22) Hehre, W. J.; Ditchfield, K.; Pople, J. A. J. Chem. Phys. 1972, 56, 2257-2261.

(23) Clark, T.; Chandrasekhar, J.; Spitznagel, G. W.; Schleyer, P. V. R. J. Comput. Chem. 1983, 4, 294-301.

(24) Von Rague Schleyer, P.; Maerker, C.; Dransfeld, A.; Jiao, H.; van Eikema Hommes, N. J. R. J. Am. Chem. Soc. 1996, 7863, 6317-6318. 